\title{
O(2) HOPF BIFURCATION OF VISCOUS SHOCK WAVES IN A CHANNEL
}

\author{
ALIN POGAN, JINGHUA YAO, AND KEVIN ZUMBRUN
}

\begin{abstract}
Extending work of Texier and Zumbrun in the semilinear non-reflection symmetric case, we study $O(2)$ transverse Hopf bifurcation, or "cellular instability", of viscous shock waves in a channel, for a class of quasilinear hyperbolic-parabolic systems including the equations of thermoviscoelasticity. The main difficulties are to (i) obtain Fréchet differentiability of the time- $T$ solution operator by appropriate hyperbolic-parabolic energy estimates, and (ii) handle $O(2)$ symmetry in the absence of either center manifold reduction (due to lack of spectral gap) or (due to nonstandard quasilinear hyperbolic-parabolic form) the requisite framework for treatment by spatial dynamics on the space of time-periodic functions, the two standard treatments for this problem. The latter issue is resolved by Lyapunov-Schmidt reduction of the time- $T$ map, yielding a four-dimensional problem with $O(2)$ plus approximate $S^{1}$ symmetry, which we treat "by hand" using direct Implicit Function Theorem arguments. The former is treated by balancing information obtained in Lagrangian coordinates with that from associated constraints. Interestingly, this argument does not apply to gas dynamics or magnetohydrodynamics (MHD), due to the infinite-dimensional family of Lagrangian symmetries corresponding to invariance under arbitrary volume-preserving diffeomorphisms.
\end{abstract}

\section{INTRODUCTION}

In this paper, we treat transverse Hopf bifurcation, or time-periodic "cellular instability", of planar viscous shock waves in an infinite channel with periodic boundary conditions, for a class of hyperbolic-parabolic systems including the equations of thermoviscoelasticity. Transverse Hopf bifurcation has been treated in [TZ2] for semilinear equations. The main differences here are partial parabolicity/lack of parabolic smoothing and reflection symmetry of the physical equations. The former adds considerable technical difficulty to do with the basic issue of regularity of the time- $T$ solution map, as discussed for the 1D case in [TZ3]. The latter implies that the underlying bifurcation is not of planar Hopf type, but, rather, a four-dimensional $O(2)$ Hopf bifurcation as discussed for example in [GSS]: roughly speaking, a "doubled" Hopf bifurcation coupled by nonlinear terms. ${ }^{1}$

$O(2)$ Hopf bifurcation is typically treated by center manifold reduction followed by transformation to a (doubly) angle invariant normal form, and thereby to a planar stationary bifurcation with $D_{4}$ symmetry in the (two) radial coordinates. Here, however, the linearized operator about the wave has no spectral gap, hence standard center manifold theorems do not apply; indeed, existence of a center manifold is unclear. Instead, we proceed by the Lyapunov-Schmidt reduction framework of [TZ2], applied to the time- $T$ evolution map of the underlying perturbation equations, resulting in a 4-dimensional stationary bifurcation problem with $O(2)$ symmetry plus an additional "approximate $S^{1}$ symmetry" induced by the underlying rotational linearized flow. The latter is then analyzed "by hand", using direct rescaling/Implicit Function Theorem arguments. ${ }^{2}$

\footnotetext{
Research of A.P. was partially supported under NSF grant no. DMS-0300487.

Research of J.Y. was partially supported under NSF grant no. DMS-0300487.

Research of K.Z. was partially supported under NSF grant no. DMS-0300487.

${ }^{1}$ Corresponding address: 831 East 3rd St, Bloomington, IN 47405, Phone: (812) 855-4591, Fax: (812) 855-0046.

${ }^{2}$ E-mail addresses: pogana@miamioh.edu (A Pogan), jinghua-yao@uiowa.edu(J. Yao), kzumbrun@indiana.edu(K.
} Zumbrun 
We note that, though there exist other methods suitable to treat related problems without spectral gap, notably the spatial dynamics approach used by Iooss, Sandstede-Scheel, and otherssee in particular [GSS, SS])- the "reverse temporal dynamics" approach of [TZ3] is the only one that has so far been successfully applied (in the 1-D case) to the viscous shock solutions of physical, partially parabolic systems; see Section 1.5 for further discussion. Indeed, the main advantage of this method is that it typically applies whenever there is an existing time-evolutionary stability theory for the background equilibrium solution, which in this case has already been developed. A disadvantage of the method is, simply, that it is not the standard one, and so, as turns out to be the case here, one cannot always appeal to existing theory to treat the resulting reduced system. Though elementary, the treatment of the nonstandard finite-dimensional reduced system is thus a significant part of our analysis.

Regarding regularity, a critical aspect, as in the 1D case [TZ3], is to work in Lagrangian rather than Eulerian coordinates, in which hyperbolic transport modes become constant-coefficient linear rather than quasilinear as in the Eulerian case, and certain key variational energy estimates do not lose derivatives. For our argument, we require also other favorable properties related to stability of constant solutions that are evident in the Eulerian formulation by existence of a convex entropy, but for multi-D are in the Lagrangian formulation are less clear. Fortunately, this issue may be addressed by a slight extension of ideas of Dafermos [Da] regarding involution and contingent entropy, in particular yielding the necessary properties for the equations of thermoviscoelasticity.

Lest one conclude that Eulerian and Lagrangian formulations share identical properties, we point out that for gas dynamics and MHD, this is far from the case. Since the stress tensor in these cases depends on the strain tensor through density alone, that is, only through the Jacobian of the displacement map, it follows that the Lagrangian equations are invariant under any volumepreserving diffeomorphism, an infinite-dimensional family of symmetries preventing asymptotic stability of constant solutions. Meanwhile, the Eulerian equations, possessing a convex entropy, automatically do have the property of asymptotic stability. This represents a genuine difference between Eulerian and Lagrangian formulations for gas dynamics and MHD, and an obstruction to the methods of this paper. We discuss in Section 5 various ideas how this might be overcomed.

Notation: In what follows $\mathbf{u}: \mathbb{R} \times[-\pi, \pi] \rightarrow \mathbb{R}^{n}$ is a smooth function, periodic on $[-\pi, \pi]$. Unless otherwise indicated, indices $j, k$ are in the range $\{1,2\}$, and summations in $j, k$ are from 1 to 2. We denote $\partial_{j}:=\frac{\partial}{\partial x_{j}}$ and $\partial_{t}:=\frac{\partial}{\partial t}, \alpha \in \mathbb{N}^{2}$, and $D^{\alpha}:=\partial_{x}^{\alpha}$. We denote the symmetric part of a matrix or linear operator $N$ by $\operatorname{Re} N:=\frac{1}{2}\left(N+N^{*}\right)$ and its spectrum by $\sigma(N)$.

1.1. Equations and assumptions. Consider a smooth one-parameter family of standing viscous planar shock solutions

$$
\mathbf{u}(x, t)=\overline{\mathbf{u}}^{\varepsilon}\left(x_{1}\right), \quad \lim _{x_{1} \rightarrow \pm \infty} \overline{\mathbf{u}}^{\varepsilon}\left(x_{1}\right)=\mathbf{u}_{ \pm}^{\varepsilon} \quad(\text { constant for fixed } \varepsilon),
$$

of a smoothly-varying family of conservation laws

$$
\begin{aligned}
\mathbf{u}_{t}=\mathcal{F}(\varepsilon ; \mathbf{u}) & :=\sum_{j k} \partial_{j}\left(\check{B}^{j k}(\varepsilon ; \mathbf{u}) \partial_{k} \mathbf{u}\right)-\sum_{j} \partial_{j} \check{F}^{j}(\varepsilon ; \mathbf{u}) \\
& =\sum_{j k} \partial_{j}\left(B^{j k}(\varepsilon ; \mathbf{u}) \partial_{k} \mathbf{u}\right)-\sum_{j} \partial_{j} F^{j}(\varepsilon ; \mathbf{u})+\mathcal{G}(\mathbf{u}, \varepsilon) m \mathbf{u} \quad \mathbf{u} \in \mathbb{R}^{n}
\end{aligned}
$$

on the periodic channel $x_{1} \in \mathbb{R}, x_{2} \in[-\pi, \pi]_{\text {per }}$ subject to (possibly vanishing) constraints

$$
m \mathbf{u}:=\sum_{j} m_{j} \partial_{j} \mathbf{u}=0, \quad m_{j}=\text { constant } \in \mathbb{R}^{q \times n}, \quad \text { for some } \quad q \in \mathbb{Z}+,
$$


preserved by the flow of (1.2), with associated linearized operators

$$
L(\varepsilon) \mathbf{v}:=\sum_{j k} \partial_{j}\left(B^{j k}\left(\varepsilon ; \overline{\mathbf{u}}^{\varepsilon}\right) \partial_{k} \mathbf{v}-\partial_{\mathbf{u}} B^{j k}\left(\varepsilon ; \overline{\mathbf{u}}^{\varepsilon}\right) \mathbf{v} \partial_{k} \overline{\mathbf{u}}^{\varepsilon}\right)-\sum_{j} \partial_{j}\left(\partial_{\mathbf{u}} F^{j}\left(\varepsilon ; \overline{\mathbf{u}}^{\varepsilon}\right) \mathbf{v}\right)+\mathcal{G}\left(\varepsilon ; \overline{\mathbf{u}}^{\varepsilon}\right) m \mathbf{v} .
$$

In the case of a nontrivial constraint $m \neq 0$, following [Da], we assume further that

$$
m L(\varepsilon)=\Gamma(\varepsilon) m
$$

for some family of second-order linear partial differential operators $\Gamma(\varepsilon)$ generating a $C^{0}$ semigroup. This is sufficient but not necessary for preservation of the condition $m \mathbf{u}=0$ under the linearized flow $\mathbf{u}_{t}=L(\varepsilon) \mathbf{u}$, by well-posedness of the induced flow $\mu_{t}=\Gamma(\varepsilon) \mu$, governing $\mu:=m \mathbf{u}$.

In most examples the constraints (1.3) are given as divergence free or curl free conditions, as for many equations of mathematical-physics; see examples 1.4 and 1.6 below. Example 1.7 does not involve a constraint. Typically, the bifurcation parameter $\varepsilon$ measures shock amplitude or other physical parameters. Here, the linear operator $L(\varepsilon)$ is considered as a closed linear operator on $L^{2}\left(\mathbb{R} \times[-\pi, \pi], \mathbb{C}^{n}\right)$ with domain $\operatorname{dom}(L(\varepsilon))=H^{2}\left(\mathbb{R} \times[-\pi, \pi], \mathbb{C}^{n}\right)$, and the functions $B^{j k}$ : $(-\delta, \delta) \times \mathbb{R}^{n} \rightarrow \mathbb{R}^{n \times n}$ and $F^{j}:(-\delta, \delta) \times \mathbb{R}^{n} \rightarrow \mathbb{R}^{n}$ are smooth in u, see also Hypothesis (H0) below. Equations (1.2) are typically shifts $B^{j k}(\varepsilon ; \mathbf{u})=B^{j k}(\mathbf{u}), F^{1}(\varepsilon ; \mathbf{u}):=f^{1}(\mathbf{u})-s(\varepsilon) \mathbf{u}$ of a single equation

$$
\mathbf{u}_{t}=\sum_{j k} \partial_{j}\left(B^{j k}(\mathbf{u}) \partial_{k} \mathbf{u}\right)-\sum_{j} \partial_{j} F^{j}(\mathbf{u})+\mathcal{G}(\mathbf{u}) m
$$

written in coordinates $\tilde{x}=\left(x_{1}-s(\varepsilon) t, x_{2}\right)$ moving with traveling-wave solutions $\mathbf{u}(x, t)=\overline{\mathbf{u}}^{\varepsilon}\left(x_{1}-\right.$ $s(\varepsilon) t)$ of varying speeds $s(\varepsilon)$. Profiles $\overline{\mathbf{u}}^{\varepsilon}$ satisfy the standing-wave ODE

$$
\check{B}^{11}(\varepsilon ; \mathbf{u}) \mathbf{u}^{\prime}=\check{F}^{1}(\varepsilon ; \mathbf{u})-\check{F}^{1}\left(\varepsilon ; \mathbf{u}_{-}^{\varepsilon}\right) .
$$

We assume, further, that there is an invertible coordinate change $\mathbf{u} \rightarrow w$ yielding the "partially symmetric hyperbolic-parabolic form" of [Z2]:

$$
\mathbb{A}^{0}(\varepsilon ; w) w_{t}=\sum_{j k} \partial_{j}\left(\mathbb{B}^{j k}(\varepsilon ; w) \partial_{k} w\right)-\sum_{j} \mathbb{A}^{j}(\varepsilon ; w) \partial_{j} w+\mathbb{G} m w+\left(\begin{array}{l}
0 \\
\tilde{g}
\end{array}\right),
$$

with $\tilde{g}=\mathcal{O}\left(\left|\nabla_{x} w\right|^{2}\right)$, together with conditions (A3) below.

We shall use the notation

$$
\begin{gathered}
A^{j}:(-\delta, \delta) \times \mathbb{R}^{n} \rightarrow \mathbb{R}^{n \times n}, \quad A^{j}(\varepsilon ; \mathbf{u})=F_{\mathbf{u}}^{j}(\varepsilon ; \mathbf{u}) ; \\
A_{ \pm}^{j}, B_{ \pm}^{j k}:(-\delta, \delta) \rightarrow \mathbb{R}^{n \times n} \\
A_{ \pm}^{j}(\varepsilon)=F_{\mathbf{u}}^{j}\left(\varepsilon ; \mathbf{u}_{ \pm}^{\varepsilon}\right)=A^{j}\left(\varepsilon ; \mathbf{u}_{ \pm}^{\varepsilon}\right), \quad B_{ \pm}^{j k}(\varepsilon)=B^{j k}\left(\varepsilon ; \mathbf{u}_{ \pm}^{\varepsilon}\right) . \\
\mathbb{A}_{ \pm}^{j}, \mathbb{B}_{ \pm}^{j k}:(-\delta, \delta) \rightarrow \mathbb{R}^{n \times n} \\
\mathbb{A}_{ \pm}^{j}(\varepsilon)=\mathbb{A}^{j}\left(\varepsilon ; w_{ \pm}^{\varepsilon}\right), \quad \mathbb{B}_{ \pm}^{j k}(\varepsilon)=\mathbb{B}^{j k}\left(\varepsilon ; w_{ \pm}^{\varepsilon}\right) .
\end{gathered}
$$

In what follows, if $A$ is an $n \times n$ matrix we will use lower subscripts for the block decomposition $A=\left(\begin{array}{ll}A_{11} & A_{12} \\ A_{21} & A_{22}\end{array}\right)$, where $A_{11}$ is an $(n-r) \times(n-r)$ matrix and $A_{22}$ is an $r \times r$ matrix. 
1.1.1. Structural conditions. We make the following structural assumptions on the equations:

(A1) For every $j, k \in\{1,2\}$ there exist functions $b^{j k}:(-\delta, \delta) \times \mathbb{R}^{n} \rightarrow \mathbb{R}^{r \times r}$ and function $\tilde{b}^{j k}$ : $(-\delta, \delta) \times \mathbb{R}^{n} \rightarrow \mathbb{R}^{r \times r}$ such that $B^{j k}$ and $\mathbb{B}^{j k}$ have the representations

$$
B^{j k}(\varepsilon ; \mathbf{u})=\left(\begin{array}{cc}
0 & 0 \\
0 & b^{j k}(\varepsilon ; \mathbf{u}),
\end{array}\right), \quad \mathbb{B}^{j k}(\varepsilon ; w)=\left(\begin{array}{cc}
0 & 0 \\
0 & \tilde{b}^{j k}(\varepsilon ; w),
\end{array}\right) .
$$

Moreover, the first $(n-r)$ components of $F^{j}(\varepsilon, \mathbf{u}), j \in\{1,2\}$ are linear in $\mathbf{u}$.

(A2) There exists a matrix-valued function $A^{0}:(-\delta, \delta) \times \mathbb{R}^{n} \rightarrow \mathbb{R}^{n \times n}$ smooth in $\mathbf{u} \in \mathbb{R}^{n}$, positive definite and having a block-diagonal structure such that

(i) $A_{11}^{0}(\varepsilon ; \mathbf{u}) A_{11}^{j}(\varepsilon ; \mathbf{u})$ is symmetric for any $\varepsilon \in(-\delta, \delta), \mathbf{u} \in \mathbb{R}^{n}$;

(ii) $A_{11}^{0}(\varepsilon ; \mathbf{u}) A_{11}^{1}(\varepsilon ; \mathbf{u})$ is either positive definite or negative definite for any $\varepsilon \in(-\delta, \delta)$, $\mathbf{u} \in \mathbb{R}^{n}$

(iii) there exists a constant $\theta>0$ such that

$$
\sum_{j, k} \mathbf{v}_{j} \cdot\left(A_{22}^{0}(\varepsilon ; \mathbf{u}) b^{j k}(\varepsilon ; \mathbf{u}) \mathbf{v}_{k}\right) \geq \theta \sum_{j}\left|\mathbf{v}_{j}\right|^{2} \quad \text { for all } \quad \mathbf{v}_{1}, \mathbf{v}_{2} \in \mathbb{R}^{r} .
$$

(A3) $\mathbb{A}^{0}$ is block-diagonal, symmetric positive definite and:

(i) $\mathbb{A}_{11}^{j}(\varepsilon ; w)$ is symmetric for any $\varepsilon \in(-\delta, \delta), w \in \mathbb{R}^{n}$;

(ii) $\mathbb{A}_{11}^{1}(\varepsilon ; w)$ is either positive definite or negative definite for any $\varepsilon \in(-\delta, \delta), w \in \mathbb{R}^{n}$;

(iii) $\mathbb{A}_{ \pm}^{j}(\varepsilon)$ is symmetric $(\bmod m)$ for any $\varepsilon \in(-\delta, \delta)$, in the sense that $\mathbb{A}_{ \pm}^{j}(\varepsilon)+v_{ \pm}^{T} m_{j}$ is symmetric for some $v_{ \pm} \in \mathbb{R}^{r \times n}$;

(iv) there exists a constant $\theta>0$ such that

$$
\sum_{j, k} \mathbf{v}_{j} \cdot\left(\mathbb{A}_{22}^{0}(\varepsilon ; w) \tilde{b}^{j k}(\varepsilon ; w) \mathbf{v}_{k}\right) \geq \theta \sum_{j}\left|\mathbf{v}_{j}\right|^{2} \text { for all } \mathbf{v}_{1}, \mathbf{v}_{2} \in \mathbb{R}^{r} ;
$$

(v) There exist skew matrices $\mathbb{K}_{ \pm}^{j}(\varepsilon) \in \mathbb{R}^{n \times n}$ such that

$$
\operatorname{Re} \sum_{j k}\left(\mathbb{K}_{ \pm}^{j}(\varepsilon)\left(\mathbb{A}_{ \pm}^{0}(\varepsilon)\right)^{-1} \mathbb{A}_{ \pm}^{k}(\varepsilon)+\mathbb{B}_{ \pm}^{j k}(\varepsilon)\right) \xi_{j} \xi_{k} \geq \theta|\xi|^{2}>0(\bmod m(\xi))
$$

for all $\xi \in \mathbb{R}^{2}$ and $\varepsilon \in(-\delta, \delta)$, in the sense that the displayed quadratic form is definite when restricted to the kernel of the symbol $m(\xi):=\sum_{j} m_{j} \xi_{j}$ of constraint $m$, or, equivalently, its sum with $C\left(m^{*} m\right)(\xi)$ is $\geq \theta$ for $C>0$ sufficiently large.

(B1) Equations (1.2) are invariant under $S: x_{2} \rightarrow-x_{2}, \mathbf{u} \rightarrow M \mathbf{u}$, for $M \in \mathbb{R}^{n \times n}$ constant, such that $M^{2}=\mathrm{Id}$.

Conditions (A1)-(A2) are analogous to the 1D conditions of [TZ3], used to obtain Fréchet differentiability of the nonlinear source term in the time- $T$ solution map for the perturbation equations of (1.2) about a standing shock, themselves strengthened versions of the standard conditions of [Z2] for systems of conservation laws with physical viscosity. Condition (A1) expresses the fact that coordinate $\mathbf{u}_{2}$ experiences second-order parabolic smoothing-i.e., the principal part of the $\mathbf{u}_{2}$ equation is a parabolic equation $\mathbf{u}_{2, t}=\sum_{j k} \partial_{j}\left(b^{j k}(\varepsilon ; \mathbf{u}) \partial_{k} \mathbf{u}_{2}\right)$ - while coordinate $\mathbf{u}_{1}$ does not- i.e., the principal part of the $\mathbf{u}_{1}$ equation is a first-order hyperbolic conservation law $\mathbf{u}_{1, t}+\sum_{j=1}^{d} A_{11}^{j}(\varepsilon ; \mathbf{u}) \partial_{j} \mathbf{u}_{1}=0$. The fact that these equations are of parabolic (hyperbolic) type follows from the block-symmetrizability assumptions (A2), where (A2)(ii) guarantees uniform ellipticity of $b^{j k}$ terms and (A2)(iii) is the familiar condition of Friedrichs symmetrizability guaranteeing hyperbolicity of $A_{11}^{0} \partial_{t}+\sum_{j=1}^{d} A_{11}^{j}(\varepsilon ; \cdot) \partial_{j}$.

The key second condition of (A1), together with the assumed block structure of $B^{j k}$, imposes further that the $\mathbf{u}_{1}$ equation be linear constant-coefficient, yielding easily Fréchet differentiability 
in $\mathbf{u}$ of the "hyperbolic" (i.e., $\mathbf{u}_{1}$ ) part of the time-evolution map, which in the quasilinear hyperbolic case would otherwise not hold (see Appendix A [TZ3] for further discussion/computations). Condition (A2)(ii) is a technical condition that is generally satisfied and has proved to be useful in all aspects of viscous shock analysis from existence of profiles to stability [Z2]. Condition (A3) is equivalent $(\bmod m)$ to the multi-D conditions of $[\mathrm{Z1}, \mathrm{Z} 2]$, used to obtain damping-type time-evolutionary energy estimates and high-frequency resolvent bounds.

To summarize: from the special linear structure of the hyperbolic part of the $\mathbf{u}$ equations, together with block hyperbolic-parabolic structure, we are able to obtain the Fréchet differentiability of the time-evolution map needed even to begin our analysis. For the high-frequency damping estimates needed to carry out our ultimate Lyapunov-Schmidt reduction argument, we require the additional symmetry information (A3)(iii), (v) at the end states of the shock. Condition (A3)(v) may be recognized as a $(\bmod m(\xi))$ version of Kawashima's condition for hyperbolic-parabolic smoothing $[\mathrm{Kaw}]$, implying in particular a $(\bmod m(\xi))$ version of his genuine coupling condition: there is no eigenvector of $\sum_{j} \mathbb{A}_{ \pm}^{j}(\varepsilon) \xi_{j}$ lying in $\operatorname{ker} \sum_{j, k} \mathbb{B}_{ \pm}^{j k}(\varepsilon) \xi_{j} \xi_{k} \cap \operatorname{ker} m(\xi)$, for any $\xi \in \mathbb{R}^{2}$. Together, (A3) (iii) and (v) imply, by the restriction to ker $m(\xi)$ of the energy estimates used by Kawashima in the unconstrained case (see, e.g., [KaS] or the proof of Lemma 3.18 [Z2]), the spectral bound

$$
\operatorname{Re} \sigma\left(L_{ \pm}(\xi ; \varepsilon)_{\mid \operatorname{ker} m(\xi)}\right) \leq-\theta \frac{|\xi|^{2}}{1+|\xi|^{2}}, \quad \xi \in \mathbb{R}^{2}
$$

on the Fourier symbol $L_{ \pm}(\xi ; \varepsilon)$ of the limiting constant-coefficient operators $L_{ \pm}(\varepsilon)$ as $x_{1} \rightarrow \pm \infty$, when restricted to its invariant subspace $\operatorname{ker} m(\xi)$.

Condition (B1) together with translation-invariance in $x_{2}$, implies $\mathrm{O}(2)$ symmetry in the perturbation equations around the (symmetric, since constant in $x_{2}$-direction) background shock solutions, with $R(\theta): \mathbf{u}\left(x_{1}, \cdot, t\right) \rightarrow \mathbf{u}\left(x_{1},(\cdot+\theta) \bmod 2 \pi, t\right)$ corresponding to rotation and $S$ reflection, $R(\theta) S=S R(-\theta)$. That is, "rotation" in this context should be thought of as $x_{2}$-translation.

Remark 1.1. With slight further effort, we may replace as in [Z1, Z2] the uniform ellipticity conditions (A2)(iii) and (A3)(iv) with the symbolic conditions $\operatorname{Re}\left(\sum_{j, k} A_{22}^{0}(\varepsilon ; \mathbf{u}) b^{j k}(\varepsilon ; \mathbf{u}) \xi_{j} \xi_{k}\right) \geq \theta|\xi|^{2}$, for all $\xi \in \mathbb{R}^{2}$, using Gärding's inequality instead of direct integration by parts in the energy estimates of Section 2; see for example the proof of [Z1, Proposition 5.9]. However, this is not needed for the physical applications from continuum mechanics that we have in mind.

1.1.2. Technical hypotheses. To (A1)-(A3), (B1), we add the following technical conditions. Here and elsewhere, $\sigma(M)$ denotes the spectrum of a matrix or linear operator $M$.

(H0) For any $j, k \in\{1,2\}$ the functions $F^{j}, B^{j k}, \mathbb{A}^{j}, \mathbb{B}^{j, k}$ are of class $\mathcal{C}^{\nu}$, for some $\nu \geq 5$.

(H1) The eigenvalues of $\mathbb{A}_{11}^{1}\left(\varepsilon ; \overline{\mathbf{u}}^{\varepsilon}\left(x_{1}\right)\right)$ have multiplicity independent of $x_{1} \in \mathbb{R}$.

(H2) $\sigma\left(\check{A}_{ \pm}^{1}(\varepsilon)\right)$ is real, semisimple, and nonzero for $\check{A}_{ \pm}^{1}(\varepsilon):=\check{F}_{\mathbf{u}}^{1}\left(\varepsilon ; \overline{\mathbf{u}}_{ \pm}^{\varepsilon}\right)$.

(H3) The Fourier symbol $\Gamma_{ \pm}(\xi ; \varepsilon)$ of the limiting constant-coefficient operators $\Gamma_{ \pm}(\varepsilon)$ as $x \rightarrow \pm \infty$ of the operator $\Gamma(\varepsilon)$ defined in $(1.5)$ satisfies $\operatorname{Re} \sigma\left(\left.\Gamma_{ \pm}(\xi ; \varepsilon)\right|_{\text {Range } m(\xi)}\right) \leq-\theta \frac{|\xi|^{2}}{1+|\xi|^{2}}$, when restricted to its invariant subspace Range $m(\xi)$, whence ${ }^{3} L_{ \pm}(\xi ; \varepsilon)$ satisfies the (unrestricted) bound

$$
\operatorname{Re} \sigma\left(L_{ \pm}(\xi ; \varepsilon)\right) \leq-\theta \frac{|\xi|^{2}}{1+|\xi|^{2}}, \quad \xi \in \mathbb{R}^{2}, \varepsilon \in(-\delta, \delta), \theta>0
$$

(H4) Considered as connecting orbits of (1.6), $\overline{\mathbf{u}}^{\varepsilon}$ lie in an $\ell$-dimensional manifold, $\ell \geq 1$, of solutions of 1.1), obtained as a transversal intersection of the unstable manifold at $\mathbf{u}_{-}^{\varepsilon}$ and the stable manifold at $\mathbf{u}_{+}^{\varepsilon}$. (In the most typical case of a Lax-type shock [L], $\ell=1$ and the manifold of solutions consists simply of the set of $x_{1}$-translates of a single wave.)

\footnotetext{
${ }^{3}$ Using (1.12) for $\mathbf{u} \in \operatorname{ker} m(\xi)$ and for all else the fact that $\left.\left(L_{ \pm}(\xi ; \varepsilon)\right)-\lambda\right) \mathbf{u}=0$ implies $\left(\Gamma_{ \pm}(\xi ; \varepsilon)-\lambda\right) m(\xi) \mathbf{u}=0$.
} 
(H5) The full (unconstrained) $1 \mathrm{D}$ system $\mathbf{u}_{t}=\partial_{1}\left(\check{B}^{11}(\varepsilon ; \mathbf{u}) \partial_{1} \mathbf{u}\right)-\partial_{1} \check{F}^{1}(\varepsilon ; \mathbf{u})$ has the structure (1.11) of (A1) with possibly larger $\check{r} \geq r$, along with the corresponding analog of (A2).

(H6) The eigenvalue equation $\left(\lambda-L_{k}(\varepsilon) \mathbf{u}=0\right.$ of the operator-valued symbol $L_{k}(\varepsilon)$ obtained from $L(\varepsilon)$ by Fourier transform with respect to the $x_{2}$ coordinate (see Section 1.3 for further details) is expressible as an equivalent first-order ODE $\partial_{1} W=R\left(x_{1}, k, \lambda ; \varepsilon\right) W$ with respect to $x_{1}$.

Hypotheses (H0)-(H5) are the standard ones imposed in the stability theory for planar viscous shock waves [Z2], and are used here to allow us to bring to bear tools from the stability literature, following the philosophy of [TZ2, TZ3]; hypothesis (H6), automatic in the case without constraints, is also needed to apply those tools. The most substantial of these conditions is (H3), which together with symmetry assumptions (A3) (iii) and (v), guarantees stability of constant solutions $\mathbf{u} \equiv \overline{\mathbf{u}}_{ \pm}^{\varepsilon}$ at dissipative rates: more precisely, that the spectra $\lambda$ of the linearized operators about these constant solutions obey bounds

$$
\operatorname{Re} \lambda \leq-c\left(|\operatorname{Im} \lambda|^{2}+k^{2}\right) /\left(d+\left(|\operatorname{Im} \lambda|^{2}+k^{2}\right)\right), \quad c, d>0,
$$

where $k$ denotes the Fourier wave number in $x_{2}$ direction. (H6) is a subtle but important hypothesis allowing us to construct the resolvent $\left(\lambda-L_{k}(\varepsilon)\right)^{-1}$ using standard ordinary-differential techniques [He]. In the case $m \equiv 0$ without a constraint, this follows automatically from (H1)-(H5).

1.2. $\mathbf{O}(2)$ Hopf bifurcation. Before stating our results, we recall the standard $O(2)$ Hopf bifurcation scenario in finite dimensions, following Crawford and Knobloch [CK]. After Center manifold/Normal form reduction, this takes the form, to cubic order, of

$$
\begin{aligned}
& \dot{z}_{1}=(\varepsilon \hat{\varkappa}(\varepsilon)+\hat{\chi}(\varepsilon) \mathrm{i}) z_{1}+\left(\hat{\Lambda}\left|z_{1}\right|^{2}+\hat{\Gamma}\left|z_{2}\right|^{2}\right) z_{1}, \\
& \dot{z}_{2}=(\varepsilon \hat{\varkappa}(\varepsilon)+\hat{\chi}(\varepsilon) \mathrm{i}) z_{2}+\left(\hat{\Lambda}\left|z_{2}\right|^{2}+\hat{\Gamma}\left|z_{1}\right|^{2}\right) z_{2},
\end{aligned}
$$

where $\varepsilon \in \mathbb{R}$ is a bifurcation parameter, $\varkappa, \chi \in \mathbb{R}$ are nonzero bifurcation coefficients, and $z_{j}, \hat{\Lambda}, \hat{\Gamma} \in$ C. Model (1.14) has $O(2)$ symmetry group consisting of rotation $R(\theta):\left(z_{1}, z_{2}\right) \rightarrow\left(z_{1} e^{\mathrm{i} \theta}, z_{2} e^{-\mathrm{i} \theta}\right)$ and reflection $S:\left(z_{1}, z_{2}\right) \rightarrow\left(z_{2}, z_{1}\right)$, with $S R(\theta)=R(-\theta) S$, and also an additional $S^{1}$ symmetry $T(\beta):\left(z_{1}, z_{2}\right) \rightarrow\left(z_{1} e^{\mathrm{i} \beta}, z_{2} e^{\mathrm{i} \beta}\right)$ associated with normal form. The linearization about the trivial equilibrium solution $\left(z_{1}, z_{2}\right)=(0,0)$ features a pair of double eigenvalues

$$
\lambda_{ \pm}(\varepsilon)=\hat{\varkappa}(\varepsilon) \varepsilon \pm \mathrm{i} \hat{\chi}(\varepsilon)
$$

crossing the imaginary axis at $\varepsilon=0$ : an equivariant Hopf bifurcation with double multiplicity forced by reflection symmetry. Noting that radial equations decouple from angular equations as

$$
\begin{aligned}
& \dot{r}_{1}=\varepsilon \hat{\varkappa}(\varepsilon) r_{1}+\left(\operatorname{Re} \hat{\Lambda}\left|r_{1}\right|^{2}+\operatorname{Re} \hat{\Gamma}\left|r_{2}\right|^{2}\right) r_{1}, \\
& \dot{r}_{2}=\varepsilon \hat{\varkappa}(\varepsilon) r_{2}+\left(\operatorname{Re} \hat{\Lambda}\left|r_{2}\right|^{2}+\operatorname{Re} \hat{\Gamma}\left|r_{1}\right|^{2}\right) r_{2},
\end{aligned}
$$

$r_{j}:=\left|z_{j}\right|$, we find that periodic solutions are exactly equilibria for the planar radial system (1.15). Under the nondegeneracy conditions

$$
\left.\operatorname{Re} \hat{\Lambda}\right|_{\varepsilon=0} \neq 0,\left.\quad \operatorname{Re}(\hat{\Lambda}+\hat{\Gamma})\right|_{\varepsilon=0} \neq 0,\left.\quad \operatorname{Re}(\hat{\Lambda}-\hat{\Gamma})\right|_{\varepsilon=0} \neq 0
$$

it is readily seen that the periodic solutions consist, besides the trivial solution $\left(r_{1}, r_{2}\right)=(0,0)$ exactly of "traveling" (or "rotating") wave solutions $\left(r_{1}, r_{2}\right) \equiv\left(r_{*}, 0\right)$ or $\left(0, r_{*}\right)$ and "standing" (or "symmetric") wave solutions $\left(r_{1}, r_{2}\right) \equiv\left(r_{*}, r_{*}\right)$ consisting of a nonlinear superposition of counterrotating traveling waves, $r_{*} \neq 0$, with associated radial bifurcations of pitchfork type $|r| \sim \sqrt{\varepsilon}$.

Restricting attention to periodic solutions with period $T(\varepsilon)$ near the linear period $T_{*}(\varepsilon):=$ $2 \pi / \hat{\chi}(\varepsilon)$, and noting that spurious radial equilibria introduced by $\operatorname{Re}(\hat{\Lambda}-\hat{\Gamma})=0$ will have different periods in $z_{1}, z_{2}$ unless $\operatorname{Im}(\hat{\Lambda}-\hat{\Gamma})=0$ as well, we find that (1.16) may be weakened to

$$
\left.\operatorname{Re} \hat{\Lambda}\right|_{\varepsilon=0} \neq 0,\left.\quad \operatorname{Re}(\hat{\Lambda}+\hat{\Gamma})\right|_{\varepsilon=0} \neq 0,\left.\quad(\hat{\Lambda}-\hat{\Gamma})\right|_{\varepsilon=0} \neq 0 .
$$


1.2.1. Alternative treatment via the displacement map. Alternatively, we show in $\S 4$ that $O(2)$ Hopf bifurcation after Lyapunov-Schmidt reduction of the time- $T$ displacement map $F_{j}:=z_{j}(T)-z_{j}(0)$ for $z_{j}(0):=a_{j}$ takes the form, to cubic order, of a two-parameter stationary bifurcation:

$$
\begin{aligned}
& 0=F_{1}\left(a_{1}, a_{2}, \varepsilon, \mu\right)=(\varepsilon \varkappa(\varepsilon, \mu)+\chi(\varepsilon) \mu \mathrm{i}) a_{1}+\left(\Lambda\left|a_{1}\right|^{2}+\Gamma\left|a_{2}\right|^{2}\right) a_{1}, \\
& 0=F_{2}\left(a_{1}, a_{2}, \varepsilon, \mu\right)=(\varepsilon \varkappa(\varepsilon, \mu)+\chi(\varepsilon) \mu \mathrm{i}) a_{2}+\left(\Lambda\left|a_{2}\right|^{2}+\Gamma\left|a_{1}\right|^{2}\right) a_{2}
\end{aligned}
$$

in four dimensions, where $a_{j}, \Lambda, \Gamma \in \mathbb{C}, \varkappa, \chi \in \mathbb{R}$ are nonzero bifurcation coefficients, and $\varepsilon, \mu \in \mathbb{R}$ are bifurcation parameters, with $\mu$ measuring the difference between $T$ and the linear period $T_{*}(\varepsilon)$. It is readily checked that, again, zeros of (1.18), corresponding to periodic solutions for the original problem, consist, besides the trivial solution $\left(a_{1}, a_{2}\right)=(0,0)$, exactly of traveling waves $\left(a_{1}, a_{2}\right)=$ $\left(a_{*}, 0\right)$ or $\left(0, a_{*}\right)$ and standing waves $\left(a_{1}, a_{2}\right)=\left(a_{*}, e^{\mathrm{i} \theta} a_{*}\right), a_{*} \neq 0, \theta \in \mathbb{R}$, each of pitchfork type $|a| \sim \sqrt{\varepsilon}$, under the nondegeneracy conditions

$$
\left.\operatorname{Re} \Lambda\right|_{\varepsilon=0} \neq 0,\left.\quad \operatorname{Re}(\Lambda+\Gamma)\right|_{\varepsilon=0} \neq 0,\left.\quad(\Lambda-\Gamma)\right|_{\varepsilon=0} \neq 0
$$

This gives a different, more direct (though higher-dimensional), route to $O(2)$ Hopf bifurcation avoiding Center Manifold or Normal form reductions, the simple form of the truncated system (1.18) being forced rather by symmetry/time-averaging. The extension to the full system then proceeds by rescaling/Implicit Function Theorem arguments, as described in $\S 4$ and Appendix B.

1.3. Statement of the main result. We are now ready to describe our main results. Note first that, by independence of coefficients of $L(\varepsilon)$ on the $x_{2}$-coordinate, the spectra of $L(\varepsilon)$ may be decomposed into spectra associated with invariant subspaces of functions $e^{\mathrm{i} k x_{2}} f\left(x_{1}\right)$ given by Fourier decomposition, on which $L(\varepsilon)$ acts as an ordinary differential operator $L_{k}(\varepsilon)$ in $x_{1}$. The operator $L_{0}(\varepsilon)$ is exactly the linearized operator for the associated one-dimensional problem, while the operators $L_{k}(\varepsilon), k \in \mathbb{Z} \backslash\{0\}$, govern the evolution of "transverse modes" with Fourier wave number $k$. See Section 3 for further discussion/computations. We perform a corresponding decomposition on the operator $\Gamma$ defined in (1.5), obtaining a family of ordinary differential operators $\Gamma_{k}(\varepsilon)$.

By the standard connection [He] between essential spectrum of asymptotically constant-coefficient ordinary differential operators and the spectra of their constant-coefficient limits as $x_{1} \rightarrow \pm \infty$, the essential spectra of $L_{k}(\varepsilon)$ are governed by the sharp bounds (1.13) implied by (A3), (H3), lying asymptotic to $\operatorname{Re} \lambda=-c / d$ for $|\lambda| \rightarrow \infty$ and bounded to the right by $\left.\operatorname{Re} \lambda \leq-c k^{2} /\left(d+k^{2}\right)\right)$, as depicted in Figure (Here, we are using also assumption (H6) that the eigenvalue equation, hence also the resolvent equation, of $L_{k}(\varepsilon)$ can be expressed as a first-order ODE, in order to apply the general result of $[\mathrm{He}]$, for which such reduction is an implicit first step- the sole point at which we use (H6).) In particular, $L_{k}(\varepsilon)$ are not sectorial operators as in the semilinear case treated in [TZ2, SS], but merely generators of $C^{0}$ semigroups, making the technical aspects of the analysisparticularly those concerned with regularity- significantly more difficult in this case.

We are interested in the case that there exists a conjugate pair of eigenvalues

$$
\lambda_{ \pm}(\varepsilon)=\gamma(\varepsilon) \pm \mathrm{i} \omega(\varepsilon), \quad \gamma^{\prime}(0), \omega(0) \neq 0
$$

crossing the imaginary axis as $\varepsilon$ crosses the bifurcation value $\varepsilon=0$, associated with transverse Fourier modes $k= \pm k_{*} \neq 0$, and that these are each of the minimal (in the presence of reflective symmetry) multiplicity two. By the assumed reflective symmetry in $x_{2}, \lambda_{+}(\varepsilon)$ and $\lambda_{-}(\varepsilon)$ are thus associated to subspaces with eigenbases

$$
e^{ \pm \mathrm{i} k_{*} x_{2}} w^{\varepsilon}\left(x_{1}\right) \text { and } e^{\mp \mathrm{i} k_{*} x_{2}} \overline{w^{\varepsilon}}\left(x_{1}\right)
$$

respectively, where "-" denotes complex conjugate. Together, they comprise a spectral transverse $O(2)$ Hopf bifurcation.

Noting that the one-dimensional operator $L_{0}(\varepsilon)$ has essential spectra passing through the origin $\lambda=0$, we see as mentioned earlier that the linearized operator $L(\varepsilon)$ has no spectral gap, preventing 
the application of standard center manifold techniques. Moreover, there is the additional technical difficulty that $L_{0}(\varepsilon)$ has at least $\ell$ embedded eigenvalues at $\lambda=0$ due to the presence of nearby shock profiles, as recorded in Hypothesis(H4), which, as zero-eigenvalues, are also resonant with bifurcation scenario (1.20). In the stability literature [Z1, Z2], such embedded eigenvalues are usefully expressed as zeros of an Evans function, or Wronskian of the linearized eigenvalue ODEs associated with the wave, a notion that extends point spectrum to allow in some cases eigenfunctions that are merely bounded and not decaying, but play a role in time-asymptotic behavior of the linearized evolution equations. As in [TZ3], we follow that convention here as well. ${ }^{4}$

Collecting the above discussion, we identify the following

Hypothesis $\left(D_{\varepsilon}\right)$. We assume that the family of operators $L(\varepsilon), \varepsilon \in(-\delta, \delta)$, satisfies:

(i) For each $\varepsilon$ there exists an open set $\Xi(\varepsilon)$ such that ${ }^{5}$

$$
\{\lambda \in \mathbb{C}: \operatorname{Re} \lambda \geq 0\} \backslash\{0\} \subset \Xi(\varepsilon) \subseteq \rho_{\mathrm{ess}}(L(\varepsilon))
$$

(ii) $\lambda=0$ is a zero of algebraic multiplicity $\ell$ (defined in (H4)) of the Evans function $D(\cdot, 0, \varepsilon)$;

(iii) There exists a pair of eigenvalues $\lambda_{ \pm}(\varepsilon)=\gamma(\varepsilon) \pm \mathrm{i} \omega(\varepsilon)$ of $L(\varepsilon)$ of multiplicity 2, for which $\gamma(0)=0$ and $\gamma^{\prime}(0)>0$, associated with operators $L_{ \pm k_{*}}(\varepsilon), k_{*} \neq 0$;

(iv) The operators $L_{k}(\varepsilon), k \in \mathbb{Z}$, have no imaginary eigenvalues other than the eigenvalues 0 and $\lambda_{ \pm}(\varepsilon)$ associated respectively with $L_{0}(\varepsilon)$ and $L_{ \pm k_{*}}(\varepsilon)$;

(v) The operators $\Gamma_{k}(\varepsilon)_{\mid \operatorname{ker} m}, k \in \mathbb{Z}$, have no eigenvalues $\operatorname{Re} \lambda \geq 0$, except possibly for $(\lambda, k)=$ $(0,0)$.

Condition $\left(D_{\varepsilon}\right)(\mathrm{i})$ follows in fact from (H3) and (A3) as discussed above, hence technically speaking is redundant; however, we include it for clarity of the spectral description. Condition $\left(D_{\varepsilon}\right)$ (ii) expresses in a generalized sense that the multiplicity- $\ell$ zero-eigenvalues guaranteed by $(\mathrm{H} 4)$, which are also embedded in the essential spectrum of $L_{0}(\varepsilon)$, are the only spectra of $L_{0}(\varepsilon)$ contained in the nonstable complex half-plane $\mathbb{C}_{+}=\{\lambda \in \mathbb{C}: \operatorname{Re} \lambda \geq 0\}$. As noted in [Z1, Z2], this is equivalent to transversality of the background shock profile as a solution of the one-dimensional traveling-wave ODE together with inviscid stability in the sense of Majda [Ma1, Ma2]. Note that this condition is expressed with respect to the unconstrained system (1.2), ignoring the condition $M \mathbf{u}=0$, so must be checked in that context, in particular taking into account the form of $G$. Condition $\left(D_{\varepsilon}\right)$ (iii) expresses $O(2)$ Hopf bifurcation while Condition $\left(D_{\varepsilon}\right)($ iv) expresses the standard accompanying nonresonance assumption apart from the zero eigenvalues of $L_{0}(\varepsilon)$ described in $\left(D_{\varepsilon}\right)$ (ii). See Figure for a pictorial illustration of this scenarios for the different values $k=0, k= \pm k_{*}$, and $k \neq 0, \pm k_{*}$. Finally, condition (v), plus the observation that $\left(L_{k}(\varepsilon)-\lambda\right) \mathbf{u}=0$ implies $\left(\Gamma_{k}(\varepsilon)-\lambda\right) m \mathbf{u}=0$, ensures that $m \mathbf{u}=0$ for any nonstable eigenvalue $\lambda$ of $L_{k}(\varepsilon)$ : in particular, that bifurcating eigenvalues $\lambda_{ \pm}(\varepsilon)$ are associated with "physical" eigenmodes satisfying constraint (1.3).

Condition $\left(D_{\varepsilon}\right)$ describes, not the standard $O(2)$ Hopf bifurcation of Section 1.2, but an " $\ell$ fold" version involving $\ell$ additional resonant eigenvalues at $\lambda=0$. Thus, we expect not isolated solutions bifurcating from a unique trivial solution, but $\ell$-dimensional families bifurcating from the $\ell$-dimensional manifold of trivial (i.e., planar) solutions given in (H4). Likewise, we expect not a single reduced equation (1.18), but a family of such systems indexed by a parameter $\mathbf{b} \in \mathbb{R}^{\ell}$.

\footnotetext{
${ }^{4}$ Existence/analyticity at $\lambda=0$ of the 1D Evans function follows from (H0)-(H5) as described, e.g., in [Z1, Z2].

${ }^{5} \rho_{\text {ess }}$ denoting the essential resolvent set, consisting of resolvent points and isolated finite-multiplicity eigenvalues.
} 
Define now the exponentially-weighted space $X_{1}^{\prime}:=H^{4}\left(\mathbb{R} \times \mathbb{T}, \mathbb{C}^{n} ; e^{\eta\left(1+\left|x_{1}\right|^{2}\right)^{1 / 2}} d x_{1} d x_{2}\right), \eta<<1$, with its natural Hilbert space norm and scalar product

$$
\begin{aligned}
\|f\|_{X_{1}^{\prime}}^{2} & \left.=\sum_{\alpha \in \mathbb{N}^{2},|\alpha| \leq 4} \int_{\mathbb{R} \times \mathbb{T}} e^{\eta\left(1+\left|x_{1}\right|^{2}\right)^{1 / 2}} \mid D^{\alpha} f\right)\left.\left(x_{1}, x_{2}\right)\right|^{2} d x_{1} d x_{2} \quad \text { and } \\
\langle f, g\rangle_{X_{1}^{\prime}} & =\sum_{\alpha \in \mathbb{N}^{2},|\alpha| \leq 4} \int_{\mathbb{R} \times \mathbb{T}} e^{\eta\left(1+\left|x_{1}\right|^{2}\right)^{1 / 2}}\left(D^{\alpha} f\right)\left(x_{1}, x_{2}\right) \cdot \overline{\left(D^{\alpha} g\right)\left(x_{1}, x_{2}\right)} d x_{1} d x_{2} .
\end{aligned}
$$

Then, the main result of this paper is:

Theorem 1.2. Let $\overline{\mathbf{u}}^{\varepsilon}, \varepsilon \in(-\delta, \delta)$, be a one-parameter family of standing viscous planar shock solutions of (1.2)-(1.3) satisfying Hypotheses (A1)-(A3), (B1), (H0)-(H6) and (DE). Then:

(i) Existence of a time-periodic solutions $\hat{u}^{\varepsilon}$ close in $X_{1}^{\prime}$ norm to $\overline{\mathbf{u}}^{\varepsilon}$ with fundamental period $T$ close to the linearized value $T_{*}(\varepsilon)=2 \pi / \omega(\varepsilon)$ is equivalent to satisfaction of one of an $\ell$-parameter family (4.11) of equations of form (1.18) plus higher-order perturbations, indexed by $\mathbf{b} \in \mathbb{R}^{\ell}$ sufficiently small, relating $\varepsilon$, a parameter $\mu$ measuring the difference between $T$ and $T_{*}(\varepsilon)$, and the projection $\left.\Pi(\varepsilon)\left(\hat{u}^{\varepsilon}-\overline{\mathbf{u}}^{\varepsilon}\right)\right|_{t=0}$, appropriately coordinatized as $\left(a_{1}, a_{2}\right) \in \mathbb{C}^{2}$, where $\Pi(\varepsilon)$ is the total eigenprojection of $L(\varepsilon)$ onto the eigenspace (1.21) associated with eigenvalues $\lambda_{ \pm}(\varepsilon){ }^{6}$

(ii) Under the genericity assumption (4.9) (Hypothesis 1, §4) analogous to (1.19), equations (1.2), (1.3) exhibit an $\ell$-fold $O(2)$-Hopf bifurcation from $\overline{\mathbf{u}}^{\varepsilon}$. Namely, the set of $X_{1}^{\prime}$-close (nontrivial) time-periodic solutions with periods nearby $T_{*}(\varepsilon)$ consists precisely of 3 smooth families indexed by b sufficiently small, of bifurcating solutions $\hat{u}^{\varepsilon, \mathbf{b}}$ satisfying $\sqrt{\varepsilon} / C \leq\left\|\hat{u}^{\varepsilon, \mathbf{b}}-\overline{\mathbf{u}}^{\varepsilon}\right\|_{X_{1}^{\prime}} \leq C \sqrt{\varepsilon}$.

The values $\left(a_{1}, a_{2}\right)$ for the families described in (ii) are $\varepsilon$-close to the "traveling-" and "standingwave" solutions $\left(a_{*}(\mathbf{b}), 0\right),\left(0, a_{*}(\mathbf{b})\right)$ and $\left(a_{\natural}(\mathbf{b}), a_{\natural}(\mathbf{b})\right)$ of the associated cubic truncated system, as described in §1.2. These are, variously, of supercritical $(\varepsilon>0)$ or subcritical $(\varepsilon<0)$ type, depending on model parameters $\Lambda(\mathbf{b}), \Gamma(\mathbf{b})$ at $\mathbf{b}=0$. In the Lax case $\ell=1$, the "traveling-wave" type solutions are actual traveling waves $\hat{u}^{\varepsilon, \mathbf{b}}(x, t)=h^{\varepsilon, \mathbf{b}}\left(x_{1}, x_{2}-d t\right)$ with respect to the transverse $x_{2}$ direction.

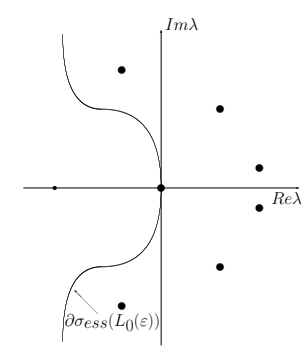

(a)

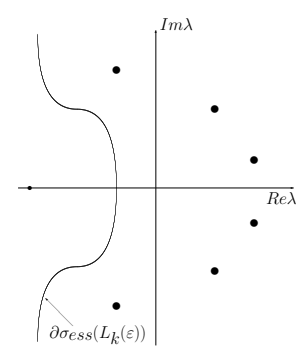

(b)

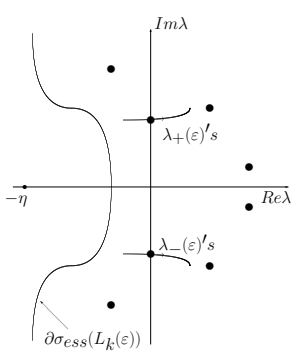

$(c)$

Figure 1. Schematic plot of spectrum of $L_{k}(\varepsilon)$ for (a) $k=0$, (b) $k \neq 0, \pm k_{*}$ and (c) $k= \pm k_{*}$. Dots denote eigenvalues of $L_{k}(\varepsilon)$, curves boundaries of $\sigma_{\text {ess }} L_{k}(\varepsilon)$.

\footnotetext{
${ }^{6}$ Here, the choice of $\mathbf{b} \in \mathbb{R}^{\ell}$ is effectively a phase condition, fixing a nearby representative from the $\ell$-fold family of profiles described in (H4).
} 
Remark 1.3. It is interesting to note that the case $\omega(0)=0$ of a transverse stationary bifurcation can be converted to the case $\omega(0) \neq 0$ of a transverse Hopf bifurcation, and vice versa, by the introduction of a moving coordinate frame $x_{2} \rightarrow x_{2}-d t$, inducing a shift $\lambda \rightarrow \lambda-\mathrm{i} k d$ in eigenvalues associated with Fourier number $k$. Setting $d=\omega(0) / k_{*}$, this converts the scenario (1.20) to that of an ordinary (non-reflective symmetric) bifurcation involving a pair of roots crossing at $\gamma(0) \pm 2 \mathrm{i} \omega(0)$ plus a pair of roots crossing at $\lambda=0$, i.e., a translationally-invariant stationary bifurcation in the moving coordinate frame. This type of bifurcation has been treated recently in $[\mathrm{M}]$ for the strictly parabolic semilinear case. Likewise, a stationary $O(2)$ bifurcation involving a double eigenvalue $\lambda(\varepsilon)=\gamma(\varepsilon)$ with wave numbers $k= \pm k_{*}$ can be converted by the change of coordinates $x_{2} \rightarrow x_{2}-d t$ to an ordinary (non- $O(2)$-symmetric) Hopf bifurcation $\lambda_{ \pm}(\varepsilon)=\gamma(\varepsilon) \pm \mathrm{i} d k_{*}$, and treated as in [TZ2] to yield a time-periodic solution in the moving coordinate frame. In the Lax case $\ell=1$, the uniqueness/shift invariance argument of $\S 4$ yields the further information that this is a traveling wave in $x_{2}$, as shown by direct (stationary) argument in [M]. Thus, there is some overlap in the results obtainable by the methods here and those of $[\mathrm{M}]$; the difference in the $O(2)$ Hopf case is that we obtain full information on all time-periodic solutions and not only traveling waves.

1.4. Applications to example systems. We now give some examples of systems coming from compressible continuum mechanics to which our results do and do not apply, in the sense that structural conditions (A), (B), (H) are or are not satisfied. We leave as a separate issue the question whether or when bifurcation conditions $\left(D_{\varepsilon}\right)$ are satisfied, to be examined numerically or analytically in specific circumstances. Accordingly, we mainly suppress the bifurcation parameter $\varepsilon$ in the discussion below.

Example 1.4. The equations of isothermal viscoelasticity in Lagrangian coordinates are

$$
\Psi_{t t}-\nabla_{X} \cdot\left(D W\left(\nabla_{X} \Psi\right)+\mathcal{Z}\left(\nabla_{X} \Psi, \nabla_{X} \Psi_{t}\right)\right)=0, \quad X \in \mathbb{R}^{d}, \quad d=2,3
$$

where $\Psi \in \mathbb{R}^{d}$ denotes deformation of an initial reference configuration of constant temperature and density, and $\nabla_{X}$. denotes divergence, taken row-wise across a matrix field. The elastic potential $W$ is a function of the deformation gradient $F:=\nabla_{X} \Psi$ and the viscous stress tensor $\mathcal{Z}$ a function of $F$ and $F_{t}$ obeying the Claussius-Duhem inequality $\mathcal{Z}(F, Q): Q \geq 0$, where ":" denotes Frobenius matrix inner product; see [A, Da, BLeZ]. Here and below, gradient and curl, like divergence are taken row-wise. We restrict attention to solutions depending on $\left(X_{1}, X_{2}\right) \in \mathbb{R} \times[-\pi, \pi)_{\text {periodic }}$.

Expressing these equations as a second-order system in $F$ and $u:=\Psi_{t}$, we obtain the system

$$
\left.F_{t}-\nabla_{X} u=0, \quad u_{t}-\nabla_{X} \cdot D W(F)=-\nabla_{X} \cdot \mathcal{Z}\left(F, \nabla_{X} u\right)\right),
$$

consisting of a first-order linear hyperbolic equation in $F$ coupled with a second-order parabolic equation in $u$, together with the constraints

$$
m(F, u):=\nabla_{X} \times F=0
$$

ensuring that $F$ is a gradient, The $d^{2}$ constraints (1.24), induced by the reduction from second- to first-order system, are not full rank, but rather have Fourier symbol $m(\xi)$ of rank $d^{2}-d$, with $d$ dimensional kernel consisting of rank-one matrices $\left\{v^{T} \xi\right\}, v \in \mathbb{R}^{d}$. Adding a term $\mathcal{G} m:=\left(\begin{array}{c}m^{*} m \\ 0\end{array}\right)$ to the righthand side, or, equivalently, substituting for (1.23)(i) the partially parabolic regularisation $F_{t}-\nabla_{X} u=\nabla_{X} \times \nabla_{X} \times F$, we arrive at a system (1.2) of form:

$$
\left.F_{t}-\nabla_{X} u=\nabla_{X} \times \nabla_{X} \times F, \quad u_{t}-\nabla_{X} \cdot D W(F)=-\nabla_{X} \cdot \mathcal{Z}\left(F, \nabla_{X} u\right)\right) .
$$

With this choice, we have $\Gamma=\operatorname{curl}^{2}$, hence, recalling that $\Delta=\nabla_{X} \operatorname{div}+\operatorname{curl}^{2}$,

$$
\Gamma_{\mid \text {ker } m}=\Delta, \quad\left(\Gamma_{k}\right)_{\mid \text {ker } m}=\partial_{1}^{2}-k^{2},
$$

from which we readily verify the conditions $(\mathrm{H} 3)$ and $\left(D_{\varepsilon}\right)(\mathrm{v})$ on $\Gamma$. 
In the commonly occurring case that the Claussius-Duhem inequality holds strictly $[\mathrm{A}]$, or

$$
Q: \mathcal{Z}(F, Q) \gtrsim \theta|Q|^{2}>0
$$

we find, examining the reduced system (1.23), that the second equation satisfies the uniform ellipticity condition (A2)(iii) and the first equation is linear, hyperbolic, hence for shock speed $c \neq 0$ we obtain (A1)-(A2) for $A^{0}=\mathrm{Id}$, with, evidently, $F$ playing the role of the hyperbolic variable and $u$ that of the parabolic variable. A more delicate point is to verify (A3), in particular symmetrizability condition (A3)(iii) for an appropriate augmented system (1.7), which amounts to symmetrizable hyperbolicity of the first-order, or inviscid, part of the equations:

$$
F_{t}-\nabla_{X} u=0, \quad u_{t}-\nabla_{X} \cdot D W(F)=0,
$$

in the vicinity of the endstates $(F, u)_{ \pm}$of the shock profile, and the Kawashima condition (A3)(v).

For, evidently the 2nd-order part of the original system (1.22) is hyperbolic if and only if $W$ is strictly rank-one convex. Likewise, hyperbolicity of (1.28) is equivalent to rank-one convexity; however, symmetrizability by a block diagonal symmetrizer is equivalent to strict convexity of $W$, which since incompatible with the principle of rotational frame-indifference cannot occur [A, BLeZ]. A related issue is existence of a convex entropy, a condition implying and in many physical situations equivalent to symmetrizable hyperbolicity [L]. It is readily verified [BLeZ, p.5] that $\eta(F, u):=$ $W(u)+|u|^{2} / 2$ is an entropy for both (1.23) and (1.28) but convex if and only if $W$ is convex.

Thus, the equations are not symmetrizable in standard sense for physically relevant elastic potentials $W$ satisfying the principles of frame indifference. However, as shown by Dafermos using the method of contingent entropies [Da, Chapter 2, pp. 30-31 and Chapter 5, pp. 111-116], the equations are block-diagonally symmetrizable $(\bmod m)$, provided that $W$ is strictly polyconvex, i.e., expressible as a strictly convex function of $\left(F, u, F^{\sharp}\right.$, $\left.\operatorname{det} F\right)$, where $F^{\sharp}$ denotes the adjugate, or transposed matrix of minors of $F$. That is, imposing the physically reasonable condition of polyconvexity of $W$, a common assumption in elasticity intermediate between between convexity and rank-one convexity $[\mathrm{B}]$, at endstates $(F, u)_{ \pm}$, we obtain satisfaction of (A3)(iii) for symmetrizers $\mathbb{A}_{ \pm}^{0}$. From these values we may then construct a global interpolant $\mathbb{A}^{0}$ by a construction similar to that of [Z2, Eg. 1.25] for the case of gas dynamics. Likewise, the Kawashima condition (A3)(v) may be verified by exhibition of explicit $(\bmod m)$ skew-symmetrizers $\mathbb{K}_{ \pm}^{j}$ for $W$ rank-one convex at $(F, u)_{ \pm}$, completing the verification of (A3). For further details/computations, see Appendix A.

Finally, (H5)-(H6) follow by inspection for shock speed $c \neq 0$, while (H4) and (D)(ii) are equivalent to the corresponding conditions for the reduced 1D problem

$$
a_{t}-u_{X}=0, \quad u_{t}-(\partial \omega / \partial a)_{X}^{T}=\left(\beta(a) u_{X}\right)_{X}, \quad a, u \in \mathbb{R}^{d}, d=2,3
$$

treated in [BLeZ], where $\omega(a):=W(F), F_{1 j}=a_{j}$, and $F_{j 2}, F_{j 3} \equiv F_{j 2}^{*}, F_{j 3}^{*}=$ constant; see again Appendix A. Summarizing, we have for $W$ polyconvex at $F_{ \pm}:=\nabla, \mathcal{Z}$ satisfying the strict ClausiusDuhem inequality, and a family of planar viscoelastic shocks $\overline{\mathbf{u}}^{\varepsilon}$ with nonzero speed $c(\varepsilon)$, satisfying (H4) and $\left(D_{\varepsilon}\right)\left(\right.$ ii) for (1.29), that $\left(D_{\varepsilon}\right)$ (iii)-(iv) imply a nonlinear O(2) transverse Hopf bifurcation as described in Theorem 1.2.

Remark 1.5. For rank-one convex $W\left(F_{ \pm}\right)$, equations $(1.28)$ admit ( $\bmod m$ ) block-diagonal symmetrizers $A_{11, \pm}^{0}(\xi)=d^{2} W\left(F_{ \pm}\right), A_{22, \pm}^{0}=\mathrm{Id}$ at endstates $(F, u)_{ \pm}$that are positive definite $(\bmod m)$. Constructing a global $A^{0}$ as a sufficiently slowly varying convex combination of $A_{ \pm}^{0}$ as we are free to do (noting that any such convex combination satisfies condition (A2)), we find by pseudodifferential techniques (specifically, Gärding's estimate) that $\left\langle v, A^{0} v\right\rangle$ is definite as a quadratic form on $L^{2}$ when restricted to ker $m$, by convexity of the symbol on $\operatorname{ker} m(\xi)$. Noting that this property is the only one needed for the high-frequency energy estimates for which assumptions (A3) are used, we thus find that the requirement of polyconvexity may be reduced to rank-one convexity at the endstates, the same (essentially minimal) condition required in the 1D analysis of [BLeZ]. 
Example 1.6. Similar considerations as in Example 1.4 yield that the equations of general (nonisothermal) thermoviscoelasticity [Da] satisfy our assumptions, so long as the thermoelastic potential $e=e(F, S)$ is a strictly convex function of $\left(F, F^{\sharp}, \operatorname{det} F\right)$ and entropy $S$, at endstates $(F, u, e)_{ \pm}$ of the shock. However, for ordinary gas dynamics, $e$ is a convex function of $\tau:=\operatorname{det} F$ and $S$ alone, where $\tau$ denotes specific volume. Thus, considered as a function of $\left(F, F^{\sharp}\right.$, $\left.\operatorname{det} F, S\right)$, it is nonstrictly convex, and indeed it is readily verified that (H4) along with (A3)(iii) fails; see $\S 5$. The constraints in both cases are $m(F, u, e):=\nabla_{X} \times F=0$ as in Example 1.4, expressing that $F$ is a gradient.

Example 1.7. Eulerian gas dynamics or MHD with artificial viscosity, i.e., strictly parabolic second-order terms, satisfy conditions (A1)-(A3), (H0)-(H6) automatically with $z(\mathbf{u})=\mathbf{u}$. This admits a much simpler treatment of regularity, as, e.g., in $[\mathrm{TZ2}, \S 6]$. For these examples, $m \equiv 0$.

1.5. Discussion and open problems. The Lyapunov-reduction/time- $T$ displacement map argument used here serves as a substitute for the Center Manifold/Normal form reduction or Lyapunovreduction/spatial dynamics methods that have been used in other contexts. It is interesting to contrast the important use of additional $S^{1}$ symmetry in these arguments, corresponding roughly to invariance with respect to time-evolution. This is imposed by force in normal form reduction, and appears naturally in the spatial dynamics approach framed in the space of time-periodic solutions. In our argument, we use the fact that time-evolution is an approximate $S^{1}$ symmetry in a similar way, to restrict the possible forms arising at the level of cubic approximation; see Remark 4.6.

An interesting open problem would be to carry out a similar analysis for physical, partially parabolic, systems either in one- or multi-dimensions using spatial dynamics techniques as done in [SS] in the one-dimensional semilinear strictly parabolic case. This appears to require both additional care in the choice of spaces/functional analytical framework, and additional analysis to establish Fréchet differentiability in the absence of parabolic smoothing of the nonlinear part of the resulting ill-posed evolution system. ${ }^{7}$ However, a possible advantage might be to remove the dependence on Lagrangian coordinates that prevents for the moment the treatment of gas dynamics and MHD. Some other ideas using the present framework are mentioned in Section 5.

We note that the same issues obstructing multi-dimensional bifurcation analysis obstruct also the proof of a multi-dimensional conditional stability result similar to that obtained in [Z5] in the onedimensional case- specifically, incompatibility between Lagrangian form needed to obtain regularity needed for the center-stable manifold reduction step and high-frequency resolvent estimates needed for time-asymptotic decay estimates- making this issue one of independent interest.

Though we do not carry it out here, spectral stability information on bifurcating solutions should be in principle available via the same reduced displacement map; indeed, it should be "reverseengineerable" from the standard normal-form analysis via the relation (through time-integration) of $\tilde{\Lambda}, \tilde{\Gamma}$ to $\Lambda, \Gamma$. A very interesting open problem is to prove a full nonlinear stability result under the assumption of spectral stability for a class of time-periodic multi-dimensional solutions including the bifurcating time-periodic waves established here, similarly as was done in one dimension in [BeSZ] in the strictly parabolic case. Another interesting direction is the treatment of spinning shocks and detonations in a cylindrical duct $[\mathrm{KS}]$, which should be treatable by similar arguments.

Finally, we note that, apart from the verification of structural conditions (A1)-(A3) and technical Hypotheses (H0)-(H6), there is the question whether and when for any particular model the spectral bifurcation scenario $\left(D_{\varepsilon}\right)$ in fact occurs. This is in general a difficult question, as is any description of the spectral properties of strongly variable-coefficient differential operators, that is typically studied either numerically (as in the related numerical Evans function study of longitudinal Hopf bifurcations in [BFZ]) or in special asymptotic limits.

\footnotetext{
${ }^{7}$ Though we think it is fairly straightforward there, we note that the latter step has so far not been carried out even for the quasilinear strictly parabolic case, and does require additional computation beyond those of [SS]
} 
As pointed out in $[\mathrm{M}]$, steady transverse $O(2)$ spectral bifurcation arises in MHD with artificial or physical viscosity, as can be seen by combining asymptotic inviscid stability computations of [FT] in the large-magnetic field limit with the observations of [ZS, Z1, Z6] relating viscous and inviscid stability in the low-frequency limit. However, so far as we know, the phenomenon of cellular instability/transverse $O(2)$ bifurcation of shock waves has so far been demonstrated either numerically or mathematically only for this single example from MHD [FT, M] and only in the steady and not the Hopf bifurcation case. The systematic cataloguing of this phenomenon for other waves and models by either numerical or analytic means we regard as an extremely interesting open problem.

Acknowledgement. Thanks to Arnd Scheel for helpful conversation improving the exposition. Thanks also to the anonymous referees for their careful reading and several helpful suggestions.

\section{VARIATIONAL ENERGY ESTIMATES}

In this section we introduce a useful energy functional associated with the perturbation equations for (1.2), and prove the key energy estimate it satisfies; see Proposition 2.2 below. We start by linearizing equation(1.2) about $\overline{\mathbf{u}}^{\varepsilon}$. The linearized equation reads as follows:

$$
\partial_{t} \mathbf{v}=L(\varepsilon) \mathbf{v}
$$

where the linear operators $L(\varepsilon)$ are defined in (1.4). Next, we note that if $\mathbf{u}$ is a solution of (1.2) and if we denote by $\mathbf{v}(x, t):=\mathbf{u}(x, t)-\overline{\mathbf{u}}^{\varepsilon}\left(x_{1}\right)$, then $\mathbf{v}$ satisfies the perturbed equation:

$$
\begin{array}{r}
\partial_{t} \mathbf{v}=L(\varepsilon) \mathbf{v}+\sum_{j k} \partial_{j}\left(B^{j k}\left(\mathbf{v}+\overline{\mathbf{u}}^{\varepsilon}\right) \partial_{k}\left(\mathbf{v}+\overline{\mathbf{u}}^{\varepsilon}\right)-B^{j k}\left(\overline{\mathbf{u}}^{\varepsilon}\right) \partial_{k} \overline{\mathbf{u}}^{\varepsilon}-B^{j k}\left(\overline{\mathbf{u}}^{\varepsilon}\right) \partial_{k} \mathbf{v}\right. \\
\left.-\partial_{\mathbf{u}} B^{j k}\left(\overline{\mathbf{u}}^{\varepsilon}\right) \mathbf{v} \partial_{k} \overline{\mathbf{u}}^{\varepsilon}\right)-\sum_{j} \partial_{j}\left(F^{j}\left(\mathbf{v}+\overline{\mathbf{u}}^{\varepsilon}\right)-F^{j}\left(\overline{\mathbf{u}}^{\varepsilon}\right)-\partial_{\mathbf{u}} F^{j}\left(\overline{\mathbf{u}}^{\varepsilon}\right) \mathbf{v}\right) .
\end{array}
$$

Using assumptions $(A 1),(A 2)$ and $(H 0)$, we infer that

$$
\begin{gathered}
\left(F^{j}\left(\mathbf{v}+\overline{\mathbf{u}}^{\varepsilon}\right)-F^{j}\left(\overline{\mathbf{u}}^{\varepsilon}\right)-\partial_{\mathbf{u}} F^{j}\left(\overline{\mathbf{u}}^{\varepsilon}\right) \mathbf{v}\right)=\left(\begin{array}{c}
0 \\
q^{j, \varepsilon}(\mathbf{v})
\end{array}\right) ; \\
B^{j k}\left(\mathbf{v}+\overline{\mathbf{u}}^{\varepsilon}\right) \partial_{k}\left(\mathbf{v}+\overline{\mathbf{u}}^{\varepsilon}\right)-B^{j k}\left(\overline{\mathbf{u}}^{\varepsilon}\right) \partial_{k} \overline{\mathbf{u}}^{\varepsilon}-B^{j k}\left(\overline{\mathbf{u}}^{\varepsilon}\right) \partial_{k} \mathbf{v}-\partial_{\mathbf{u}} B^{j k}\left(\overline{\mathbf{u}}^{\varepsilon}\right) \mathbf{v} \partial_{k} \overline{\mathbf{u}}^{\varepsilon}=\left(\begin{array}{c}
0 \\
p^{j, \varepsilon}(\mathbf{v})
\end{array}\right) .
\end{gathered}
$$

The functions $p^{j k, \varepsilon}, q^{j, \varepsilon}, j, k=1,2$, are defined as follows: for $\mathbf{v} \in H^{s}\left(\mathbb{R} \times[-\pi, \pi], \mathbb{C}^{n}\right), s \geq 2$,

$$
\begin{gathered}
q^{j, \varepsilon}(\mathbf{v})=\int_{0}^{1}(1-t) \partial_{\mathbf{u}}^{2} F_{2}^{j}\left(\overline{\mathbf{u}}^{\varepsilon}+t \mathbf{v}\right) \mathbf{v} \mathbf{v} d t \\
p^{j k, \varepsilon}(\mathbf{v})=\int_{0}^{1} \partial_{\mathbf{u}} b^{j k}\left(\overline{\mathbf{u}}^{\varepsilon}+t \mathbf{v}\right) \mathbf{v} \partial_{k} \mathbf{v}_{2} d t+\int_{0}^{1}(1-t) \partial_{\mathbf{u}}^{2} b^{j k}\left(\overline{\mathbf{u}}^{\varepsilon}+t \mathbf{v}\right) \mathbf{v} \mathbf{v} \partial_{k} \overline{\mathbf{u}}_{2}^{\varepsilon} d t .
\end{gathered}
$$

Using the functions introduced in (2.5) and (2.6), we obtain from (2.1), (2.3) and (2.4) that the perturbed equation $(2.2)$ can be written as

$$
\mathbf{v}_{t}-L(\varepsilon) \mathbf{v}=\sum_{j, k} \partial_{j}\left(\begin{array}{c}
0 \\
p^{j k, \varepsilon}(\mathbf{v})
\end{array}\right)-\sum_{j} \partial_{j}\left(\begin{array}{c}
0 \\
q^{j, \varepsilon}(\mathbf{v})
\end{array}\right) .
$$

We point out that in equation (2.7) the first block-component does not have nonlinear terms, which is a direct consequence of assumption $(A 1)$, in particular the lack of parabolic structure of the first block-component. This is an extremely useful property, actually the main reason why the smoothness issue does not cause problem later in our analysis. 
We assume throughout that the initial perturbation lies in ker $m$, hence, by invariance of ker $m$ under time-evolution, the perturbation lies always in ker $m$ and we may apply our $(\bmod m)$ structural assumptions without care. By standard energy estimates, given any $T>0$ and $s \geq 2$, there exists $\delta_{*}(T)>0$, such that, if $\left\|\mathbf{v}_{0}\right\|_{H^{s}} \leq \delta_{*}(T)$, the perturbation system has a unique solution $\mathbf{v} \in \mathcal{C}^{0}\left([0, T], H^{s}\left(\mathbb{R} \times[-\pi, \pi], \mathbb{C}^{n}\right)\right)$, with $\mathbf{v}(\cdot, 0)=\mathbf{v}_{0}$, and the bound

$$
\|\mathbf{v}(\cdot, t)\|_{H^{s}} \leq C\left\|\mathbf{v}_{0}\right\|_{H^{s}}
$$

holds for any $t \in[0, T]$ and some $C>0$ that depends on $T$, but neither on $\varepsilon$ nor on $t$. Likewise, we have a formally quadratic linearized truncation error $\left|q^{j, \varepsilon}(\mathbf{v})\right|,\left|p^{j k, \varepsilon}(\mathbf{v})\right|=O\left(|\mathbf{v}|\left(|\mathbf{v}|+\left|\nabla_{x} \mathbf{v}\right|\right)\right)$ for $\mathbf{v} \in H^{s}\left(\mathbb{R} \times[-\pi, \pi], \mathbb{C}^{n}\right)$ with $\|\mathbf{v}\|_{H^{s}} \leq C$. Our goal in this section is to establish a quadratic bound on the linearization error:

$$
\left\|\mathbf{v}(\cdot, T)-e^{L(\varepsilon) T} \mathbf{v}_{0}\right\|_{H^{s}} \leq C\left\|\mathbf{v}_{0}\right\|_{H^{s}}^{2} .
$$

Here $\left\{e^{L(\varepsilon) t}\right\}_{t \geq 0}$ denotes the $C_{0}$-semigroup generated by $L(\varepsilon)$, see, e.g., [Lun, Z1, Z2]. This inequality is far from evident in the absence of parabolic smoothing as shown in [TZ3]. The corresponding bound does not hold for quasilinear hyperbolic equations, nor as discussed in [TZ3, Appendix A], for systems of general hyperbolic-parabolic type, due to loss of derivatives. However, it follows easily for systems satisfying assumptions (A1)-(A2).

Applying the differential operator $D^{\alpha}$ to the perturbation system and multiplying the result system by $A^{0, \varepsilon}:=\left(\begin{array}{cc}A_{11}^{0}\left(\overline{\mathbf{u}}^{\varepsilon}\right) & 0 \\ 0 & A_{22}^{0}\left(\overline{\mathbf{u}}^{\varepsilon}\right)\end{array}\right):=\left(\begin{array}{cc}A_{11}^{0} & 0 \\ 0 & A_{22}^{0}\end{array}\right)$, we obtain that

$$
\begin{aligned}
A^{0, \varepsilon} D^{\alpha} \partial_{t} \mathbf{v}=A^{0, \varepsilon} & D^{\alpha} \sum_{j k} \partial_{j}\left(B^{j k}\left(\overline{\mathbf{u}}^{\varepsilon}\right) \partial_{k} \mathbf{v}\right)+A^{0, \varepsilon} D^{\alpha} \sum_{j k} \partial_{j}\left(\partial_{\mathbf{u}} B^{j k}\left(\overline{\mathbf{u}}^{\varepsilon}\right) \mathbf{v} \partial_{k} \overline{\mathbf{u}}^{\varepsilon}\right) \\
& -A^{0, \varepsilon} D^{\alpha} \sum_{j} \partial_{j}\left(A^{j} \mathbf{v}\right)-A^{0, \varepsilon} D^{\alpha} \sum_{j} \partial_{j}\left(\begin{array}{c}
0 \\
q^{j, \varepsilon}(\mathbf{v})
\end{array}\right) \\
& +A^{0, \varepsilon} D^{\alpha} \sum_{j, k} \partial_{j}\left(\begin{array}{c}
0 \\
p^{j k, \varepsilon}(\mathbf{v})
\end{array}\right) .
\end{aligned}
$$

To prove our energy estimate we need the following identities:

$$
\begin{aligned}
A^{0, \varepsilon} D^{\alpha} \partial_{j}\left(B^{j k}\left(\overline{\mathbf{u}}^{\varepsilon}\right) \partial_{k} \mathbf{v}\right)= & \partial_{j}\left(A_{22}^{0, \varepsilon} b^{j k}\left(\overline{\mathbf{u}}^{\varepsilon}\right) D^{\alpha} \partial_{k} \mathbf{v}_{2}\right)-\left(\partial_{j} A_{22}^{0, \varepsilon}\right) b^{j k}\left(\overline{\mathbf{u}}^{\varepsilon}\right) D^{\alpha} \partial_{k} \mathbf{v}_{2} \\
& +A_{22}^{0, \varepsilon} \partial_{j}\left(\sum_{\beta \leq \alpha ;|\beta|=1}\left(\begin{array}{l}
\alpha \\
\beta
\end{array}\right) D^{\beta} b^{j k}\left(\mathbf{u}^{\varepsilon}\right) D^{\alpha-\beta} \partial_{k} \mathbf{v}_{2}\right) \\
& +A_{22}^{0, \varepsilon} \partial_{j}\left(\sum_{\beta \leq \alpha ;|\beta|>1}\left(\begin{array}{c}
\alpha \\
\beta
\end{array}\right) D^{\beta} b^{j k}\left(\mathbf{u}^{\varepsilon}\right) D^{\alpha-\beta} \partial_{k} \mathbf{v}_{2}\right) \\
A^{0, \varepsilon} D^{\alpha} \partial_{j}\left(A^{j} \mathbf{v}\right)= & A^{0, \varepsilon} A^{j} D^{\alpha} \partial_{j} \mathbf{v}+A^{0, \varepsilon} D^{\alpha}\left(\partial_{j} A^{j} \mathbf{v}\right) \\
& +A^{0, \varepsilon} \sum_{\beta \leq \alpha ;|\beta| \geq 1}\left(\begin{array}{c}
\alpha \\
\beta
\end{array}\right) D^{\beta} A^{j} D^{\alpha-\beta} \partial_{j} \mathbf{v} .
\end{aligned}
$$

Next, we briefly mention the weak Moser inequality in a channel, another tool needed in our analysis. If $\kappa \geq 1, \alpha_{1}, \ldots, \alpha_{m}$ are multi-indexes, $s=\sum_{i=1}^{m}\left|\alpha_{i}\right|$ and $h_{1}, \ldots, h_{m} \in H^{\max \{s, \kappa\}}\left(\mathbb{R} \times[-\pi, \pi], \mathbb{C}^{n}\right)$, then

$$
\left\|\left(\partial^{\alpha_{1}} h_{1}\right) \cdots\left(\partial^{\alpha_{m}} h_{m}\right)\right\|_{L^{2}} \leq\left(\sum_{i=1}^{m}\left\|h_{i}\right\|_{H^{s}}\right)\left(\prod_{j \neq i}\left\|\hat{h}_{j}\right\|_{L^{1}}\right) \leq C\left(\sum_{i=1}^{m}\left\|h_{i}\right\|_{H^{s}}\right)\left(\prod_{j \neq i}\left\|h_{j}\right\|_{H^{\kappa}}\right) .
$$


The proof of (2.12) is based on the Hausdorff-Young inequality and the strong Sobolev embedding principle, see, e.g., [Z2, Lemma 1.5] for the whole-space case. As an application of the weak Moser inequality, we can prove the following lemma.

Lemma 2.1. Assume Hypotheses (A1)-(A2) and (H0). Then, for any multi-index $\alpha \in \mathbb{N}^{2}$ with $|\alpha| \geq 2$ and any $\mathbf{v} \in H^{|\alpha|}\left(\mathbb{R} \times[-\pi, \pi], \mathbb{C}^{n}\right)$ with $\mathbf{v}_{2} \in H^{|\alpha|+1}\left(\mathbb{R} \times[-\pi, \pi], \mathbb{C}^{r}\right)$, the functions $D^{\alpha} q^{j, \varepsilon}(\mathbf{v})$ and $D^{\alpha} p^{j k, \varepsilon}(\mathbf{v})$ belong to $L^{2}\left(\mathbb{R} \times[-\pi, \pi], \mathbb{C}^{n}\right)$ and the following estimate of their norms holds:

$$
\max \left\{\left\|D^{\alpha} q^{j, \varepsilon}(\mathbf{v})\right\|_{L^{2}},\left\|D^{\alpha} p^{j k, \varepsilon}(\mathbf{v})\right\|_{L^{2}}\right\} \lesssim\left(\|\mathbf{v}\|_{H^{|\alpha|}}+\left\|\mathbf{v}_{2}\right\|_{H^{|\alpha|+1}}\right)\left(\|\mathbf{v}\|_{H^{|\alpha|} \mid}+\|\mathbf{v}\|_{H^{|\alpha|} \mid}^{|\alpha|}\right) .
$$

The proof of the lemma follows directly from the week Moser inequality, (2.12), and the properties of the functions $F^{j}$ and $b^{j k}, j, k \in\{1,2\}$, stated in Section 1.1, see, e.g., [Ta, TZ3, Z2]. The main result of this section reads as follows:

Proposition 2.2. Assume Hypotheses (A1)-(A2) and (H0). Then for any $2 \leq s \leq \nu-1, T_{0}>0$, there exists some $C=C\left(T_{0}\right)>0$ such that for any $\mathbf{v}$ satisfying $(2.7)$ with initial data $\mathbf{v}(\cdot, 0)=\mathbf{v}_{0}$ sufficiently small in $H^{s}\left(\mathbb{R} \times[-\pi, \pi], \mathbb{R}^{n}\right) \cap \operatorname{ker} m$, the following inequalities hold true:

$$
\begin{gathered}
\|\mathbf{v}(\cdot, T)\|_{H^{s}}^{2}+\int_{0}^{T}\left\|\mathbf{v}_{2}(\cdot, t)\right\|_{H^{s+1}}^{2} d t \leq C\left\|\mathbf{v}_{0}\right\|_{H^{s}}^{2} \quad \text { for all } \quad T \in\left[0, T_{0}\right], \\
\left\|\mathbf{v}(\cdot, T)-e^{L(\varepsilon) T} \mathbf{v}_{0}\right\|_{H^{s}} \leq C\left\|\mathbf{v}_{0}\right\|_{H^{s}}^{2} \quad \text { for all } \quad T \in\left[0, T_{0}\right] .
\end{gathered}
$$

Proof. As noted above, from the assumption that $\mathbf{v}$ lies initially in ker $m$, we obtain that $\mathbf{v}$ lies always in $\operatorname{ker} m$, and so the $(\bmod m)$ assumptions of symmetrizability and Kawashima skewsymmetrizability may be applied; we use this freely in the argument below. Since $A^{0, \varepsilon}$ is symmetric and positive definite, we obtain that the energy functional

$$
\mathcal{E}(\mathbf{v}):=\frac{1}{2} \sum_{|\alpha| \leq s}\left\langle D^{\alpha} \mathbf{v}, A^{0, \varepsilon} D^{\alpha} \mathbf{v}\right\rangle_{L^{2}}
$$

defines a norm equivalent to $\|\cdot\|_{H^{s}}$, i.e., $\mathcal{E}(\cdot)^{1 / 2} \sim\|\cdot\|_{H^{s}}$. Using (2.10), it follows that

$$
\partial_{t} \mathcal{E}(\mathbf{v})=\sum_{|\alpha| \leq s}\left\langle D^{\alpha} \mathbf{v}, A^{0, \varepsilon} D^{\alpha} \partial_{t} \mathbf{v}\right\rangle_{L^{2}}
$$

Next, we estimate the right-hand side of (2.16), using (2.2), (2.10) and (2.11) . We break this long estimate into three separate parts. Using the hypotheses from Section 1.1 we have that for any $\mathbf{v} \in H^{s}\left(\mathbb{R} \times[-\pi, \pi], \mathbb{R}^{n}\right)$ with $\|v\|_{H^{s}} \leq \delta_{0} \leq \delta_{*}\left(T_{0}\right)$ the following holds:

$$
\begin{aligned}
& \sum_{j k} \sum_{|\alpha| \leq s}\left\langle D^{\alpha} \mathbf{v}, A^{0, \varepsilon} D^{\alpha} \partial_{j}\left(B^{j k}\left(\overline{\mathbf{u}}^{\varepsilon}\right) \partial_{k} \mathbf{v}\right)\right\rangle_{L^{2}}=\sum_{j k} \sum_{|\alpha| \leq s}\left\langle D^{\alpha} \mathbf{v}_{2}, \partial_{j}\left(A_{22}^{0, \varepsilon} b^{j k}\left(\overline{\mathbf{u}}^{\varepsilon}\right) D^{\alpha} \partial_{k} \mathbf{v}_{2}\right)\right\rangle_{L^{2}} \\
& \quad+\delta_{0}\left\|\mathbf{v}_{2}\right\|_{H^{s+1}}^{2}+C\left(\delta_{0}\right)\left\|\mathbf{v}_{2}\right\|_{H^{s}}^{2}+\mathcal{O}(1)\|\mathbf{v}\|_{H^{s}}^{2} \\
& \quad=-\sum_{j k} \sum_{|\alpha| \leq s}\left\langle D^{\alpha} \partial_{j} \mathbf{v}_{2},\left(A_{22}^{0, \varepsilon} b^{j k}\left(\overline{\mathbf{u}}^{\varepsilon}\right) D^{\alpha} \partial_{k} \mathbf{v}_{2}\right)\right\rangle_{L^{2}}+\delta_{0}\left\|\mathbf{v}_{2}\right\|_{H^{s+1}}^{2}+C\left(\delta_{0}\right)\left\|\mathbf{v}_{2}\right\|_{H^{s}}^{2}+\mathcal{O}(1)\|\mathbf{v}\|_{H^{s}}^{2} \\
& \quad \leq-\theta\left\|\mathbf{v}_{2}\right\|_{H^{s+1}}^{2}+\delta_{0}\left\|\mathbf{v}_{2}\right\|_{H^{s+1}}^{2}+C\left(\delta_{0}\right)\left\|\mathbf{v}_{2}\right\|_{H^{s}}^{2}+\mathcal{O}(1)\|\mathbf{v}\|_{H^{s}}^{2} \\
& \quad \leq\left(-\theta+\delta_{0}\right)\left\|\mathbf{v}_{2}\right\|_{H^{s+1}}^{2}+\left(C\left(\delta_{0}\right)+\mathcal{O}(1)\right)\|\mathbf{v}\|_{H^{s}}^{2} .
\end{aligned}
$$


In addition, one readily checks that

(2.18)

$$
\begin{gathered}
\sum_{j k} \sum_{|\alpha| \leq s}\left\langle D^{\alpha} \mathbf{v}, A^{0, \varepsilon} D^{\alpha} \partial_{j}\left(\partial_{\mathbf{u}} B^{j k}\left(\overline{\mathbf{u}}^{\varepsilon}\right) \mathbf{v} \partial_{k} \overline{\mathbf{u}}^{\varepsilon}\right)\right\rangle_{L^{2}}=\sum_{j k} \sum_{|\alpha| \leq s}\left\langle D^{\alpha} \mathbf{v}_{2}, A_{22}^{0, \varepsilon} D^{\alpha} \partial_{j}\left(\partial_{\mathbf{u}} b^{j k}\left(\overline{\mathbf{u}}^{\varepsilon}\right) \mathbf{v} \partial_{k} \overline{\mathbf{u}}_{2}^{\varepsilon}\right)\right\rangle_{L^{2}} \\
=\sum_{j k} \sum_{|\alpha| \leq s}\left\langle\partial_{j}\left(\left(A_{22}^{0, \varepsilon}\right)^{*} D^{\alpha} \mathbf{v}_{2}\right), D^{\alpha}\left(\partial_{\mathbf{u}} b^{j k}\left(\overline{\mathbf{u}}^{\varepsilon}\right) \mathbf{v} \partial_{k} \overline{\mathbf{u}}_{2}^{\varepsilon}\right)\right\rangle_{L^{2}} \\
\leq \mathcal{O}(1)\|\mathbf{v}\|_{H^{s}}^{2}+\delta_{0}\left\|\mathbf{v}_{2}\right\|_{H^{s+1}}^{2}+C\left(\delta_{0}\right)\|\mathbf{v}\|_{H^{s}}^{2} .
\end{gathered}
$$

Moreover, we infer that

(2.19)

$$
\begin{aligned}
\sum_{j} & \sum_{|\alpha| \leq s}\left\langle D^{\alpha} \mathbf{v}, A^{0, \varepsilon} D^{\alpha} \partial_{j}\left(A^{j} \mathbf{v}\right)\right\rangle_{L^{2}}=\sum_{j} \sum_{|\alpha| \leq s}\left\langle D^{\alpha} \mathbf{v}, A^{0, \varepsilon} A^{j} D^{\alpha} \partial_{j} \mathbf{v}\right\rangle_{L^{2}}+\mathcal{O}(1)\|\mathbf{v}\|_{H^{|\alpha|}}^{2} \\
= & \sum_{j} \sum_{|\alpha| \leq s}\left\langle D^{\alpha} \mathbf{v}_{1}, A_{11}^{0, \varepsilon} A_{11}^{j} D^{\alpha} \partial_{j} \mathbf{v}_{1}\right\rangle_{L^{2}}+\sum_{j} \sum_{|\alpha| \leq s}\left\langle D^{\alpha} \mathbf{v}_{1}, A_{11}^{0, \varepsilon} A_{12}^{j} D^{\alpha} \partial_{j} \mathbf{v}_{2}\right\rangle_{L^{2}}+\mathcal{O}(1)\|\mathbf{v}\|_{H^{|\alpha|}}^{2} \\
& +\sum_{j} \sum_{|\alpha| \leq s}\left\langle D^{\alpha} \mathbf{v}_{2}, A_{11}^{0, \varepsilon} A_{21}^{j} D^{\alpha} \partial_{j} \mathbf{v}_{1}\right\rangle_{L^{2}}+\sum_{j} \sum_{|\alpha| \leq s}\left\langle D^{\alpha} \mathbf{v}_{2}, A_{11}^{0, \varepsilon} A_{22}^{j} D^{\alpha} \partial_{j} \mathbf{v}_{2}\right\rangle_{L^{2}} \\
= & \sum_{j} \sum_{|\alpha| \leq s}-\frac{1}{2}\left\langle D^{\alpha} \mathbf{v}_{1}, \partial_{j}\left(A_{11}^{0, \varepsilon} A_{11}^{j}\right) D^{\alpha} \mathbf{v}_{1}\right\rangle_{L^{2}}+\sum_{j} \sum_{|\alpha| \leq s}\left\langle D^{\alpha} \mathbf{v}_{1}, A_{11}^{0, \varepsilon} A_{12}^{j} D^{\alpha} \partial_{j} \mathbf{v}_{2}\right\rangle_{L^{2}}+\mathcal{O}(1)\|\mathbf{v}\|_{H^{s}}^{2} \\
& +\sum_{j} \sum_{|\alpha| \leq s}\left\langle\partial_{j}\left(\left(A_{11}^{0, \varepsilon} A_{21}^{j}\right)^{*} D^{\alpha} \mathbf{v}_{2}\right), D^{\alpha} \mathbf{v}_{1}\right\rangle_{L^{2}}+\sum_{j} \sum_{|\alpha| \leq s}\left\langle D^{\alpha} \mathbf{v}_{2}, A_{11}^{0, \varepsilon} A_{22}^{j} D^{\alpha} \partial_{j} \mathbf{v}_{2}\right\rangle_{L^{2}} \\
\leq & \mathcal{O}(1)\left\|\mathbf{v}_{1}\right\|_{H^{s}}^{2}+\delta_{0}\left\|\mathbf{v}_{2}\right\|_{H^{s+1}}^{2}+C\left(\delta_{0}\right)\left\|\mathbf{v}_{1}\right\|_{H^{s}}^{2}+\delta_{0}\left\|\mathbf{v}_{2}\right\|_{H^{s+1}}^{2}+C\left(\delta_{0}\right)\|\mathbf{v}\|_{H^{s}}^{2} \\
& +\delta_{0}\left\|\mathbf{v}_{2}\right\|_{H^{s+1}}^{2}+C\left(\delta_{0}\right)\left\|\mathbf{v}_{2}\right\|_{H^{s}}^{2}+\mathcal{O}(1)\|\mathbf{v}\|_{H^{s}}^{2} \leq 3 \delta_{0}\left\|\mathbf{v}_{2}\right\|_{H^{s+1}}^{2}+C\left(\delta_{0}\right)\|\mathbf{v}\|_{H^{s}}^{2} .
\end{aligned}
$$

Next, we introduce the function $Q^{\varepsilon}: H^{s}\left(\mathbb{R} \times[-\pi, \pi], \mathbb{R}^{n}\right) \rightarrow L^{2}\left(\mathbb{R} \times[-\pi, \pi], \mathbb{R}^{n}\right)$ defined by

$$
Q^{\varepsilon}(\mathbf{v}):=-A^{0, \varepsilon} D^{\alpha} \sum_{j} \partial_{j}\left(\begin{array}{c}
0 \\
q^{j, \varepsilon}(\mathbf{v})
\end{array}\right)+A^{0, \varepsilon} D^{\alpha} \sum_{j, k} \partial_{j}\left(\begin{array}{c}
0 \\
p^{j k, \varepsilon}(\mathbf{v})
\end{array}\right) .
$$

From Lemma 2.1, we conclude that for any $\mathbf{v} \in H^{s}\left(\mathbb{R} \times[-\pi, \pi], \mathbb{R}^{n}\right)$ the following estimate holds:

$$
\begin{aligned}
& \sum_{|\alpha| \leq s}\left\langle D^{\alpha} \mathbf{v}, Q^{\varepsilon}(\mathbf{v})\right\rangle_{L^{2}}=\sum_{|\alpha| \leq s}\left\langle D^{\alpha} \mathbf{v}_{2}, Q^{\varepsilon}(\mathbf{v})\right\rangle_{L^{2}} \\
& \quad \leq \mathcal{O}(1)\left\|\mathbf{v}_{2}\right\|_{H^{s+1}}\left(\|\mathbf{v}\|_{H^{s}}+\left\|\mathbf{v}_{2}\right\|_{H^{s+1}}\right)\left(\|\mathbf{v}\|_{H^{s}}+\|\mathbf{v}\|_{H^{s}}^{s}\right) .
\end{aligned}
$$

Finally, from (2.17)-(2.21) we obtain that

$$
\begin{aligned}
\partial_{t} \mathcal{E}(\mathbf{v}) \leq & \mathcal{O}(1)\left\|\mathbf{v}_{2}\right\|_{H^{s+1}}\left(\|\mathbf{v}\|_{H^{s}}+\left\|\mathbf{v}_{2}\right\|_{H^{s+1}}\right)\left(\|\mathbf{v}\|_{H^{s}}+\|\mathbf{v}\|_{H^{s}}^{s}\right) \\
& -\theta\left\|\mathbf{v}_{2}\right\|_{H^{s+1}}^{2}+5 \delta_{0}\left\|\mathbf{v}_{2}\right\|_{H^{s+1}}^{2}+\mathcal{O}(1)\|\mathbf{v}\|_{H^{s}}^{2} .
\end{aligned}
$$

We choose $\delta_{0}>0$ such that $5 \delta_{0}<\theta / 2$. So long as $\|\mathbf{v}\|_{H^{s}}$ remains sufficiently small, we infer that

$$
\begin{aligned}
\partial_{t} \mathcal{E}(\mathbf{v}) & \leq-(\theta / 2)\left\|\mathbf{v}_{2}\right\|_{H^{s+1}}^{2}+O\left(\|\mathbf{v}\|_{H^{s}}^{2}\right) \\
& \leq-(\theta / 2)\left\|\mathbf{v}_{2}\right\|_{H^{s+1}}^{2}+C \mathcal{E}(\mathbf{v}),
\end{aligned}
$$

from which (2.13) follows by Gronwall's inequality since

$$
\mathcal{E}(\mathbf{v}(T))+(\theta / 2) \int_{0}^{T}\left\|\mathbf{v}_{2}(\cdot, t)\right\|_{H^{s+1}}^{2} d t \leq C_{2} \mathcal{E}\left(\mathbf{v}_{0}\right) .
$$


To prove (2.14), we first note that the error function $E(x, t):=\mathbf{v}(x, t)-e^{L^{\varepsilon} t} \mathbf{v}_{0}(x)$ satisfies the equation

$$
\partial_{t} E=L(\varepsilon) E-\sum_{j} \partial_{j}\left(\begin{array}{c}
0 \\
q^{j, \varepsilon}(\mathbf{v})
\end{array}\right)+\sum_{j, k} \partial_{j}\left(\begin{array}{c}
0 \\
p^{j k, \varepsilon}(\mathbf{v})
\end{array}\right)
$$

with initial condition $E(\cdot, 0)=0$. We note that equation (2.24) has a structure similar to that of (2.2). Using the same argument as in (2.10) and (2.11) one can show that

$$
\begin{aligned}
A^{0, \varepsilon} D^{\alpha} \partial_{t} E= & A^{0, \varepsilon} D^{\alpha}\left[\sum_{j k} \partial_{j}\left(B^{j k}\left(\overline{\mathbf{u}}^{\varepsilon}\right) \partial_{k} E\right)+\sum_{j k} \partial_{j}\left(\partial_{\mathbf{u}} B^{j k}\left(\overline{\mathbf{u}}^{\varepsilon}\right) E \partial_{k} \overline{\mathbf{u}}^{\varepsilon}\right)-\sum_{j} \partial_{j}\left(A^{j} E\right)\right. \\
& \left.-\sum_{j} \partial_{j}\left(\begin{array}{c}
0 \\
\left.q^{j, \varepsilon}(\mathbf{v})\right)
\end{array}\right)+\sum_{j, k} \partial_{j}\left(\begin{array}{c}
0 \\
p^{j k, \varepsilon}(\mathbf{v})
\end{array}\right)\right] \\
= & \sum_{j k} A^{0, \varepsilon} D^{\alpha} \partial_{j}\left(B^{j k}\left(\overline{\mathbf{u}}^{\varepsilon}\right) \partial_{k} E\right)+\sum_{j k} A^{0, \varepsilon} D^{\alpha} \partial_{j}\left(\partial_{\mathbf{u}} B^{j k}\left(\overline{\mathbf{u}}^{\varepsilon}\right) E \partial_{k} \overline{\mathbf{u}}^{\varepsilon}\right) \\
& -\sum_{j} A^{0, \varepsilon} D^{\alpha} \partial_{j}\left(A^{j} E\right)-\sum_{j} A^{0, \varepsilon} D^{\alpha} \partial_{j}\left(\begin{array}{c}
0 \\
q^{j, \varepsilon}(\mathbf{v})
\end{array}\right)+\sum_{j, k} A^{0, \varepsilon} D^{\alpha} \partial_{j}\left(\begin{array}{c}
0 \\
p^{j k, \varepsilon}(\mathbf{v})
\end{array}\right) \\
= & \sum_{j k} A^{0, \varepsilon} D^{\alpha} \partial_{j}\left(B^{j k}\left(\overline{\mathbf{u}}^{\varepsilon}\right) \partial_{k} E\right)+\sum_{j k} A^{0, \varepsilon} D^{\alpha} \partial_{j}\left(\partial_{\mathbf{u}} B^{j k}\left(\overline{\mathbf{u}}^{\varepsilon}\right) E \partial_{k} \overline{\mathbf{u}}^{\varepsilon}\right) \\
& -\sum_{j} A^{0, \varepsilon} D^{\alpha} \partial_{j}\left(A^{j} E\right)-Q^{\varepsilon}(\mathbf{v}) .
\end{aligned}
$$

From the definition of the energy functional in (2.15) we note that $\mathcal{E}(E) \sim\|E\|_{H^{s}}^{2}$. In addition, its time evolution $\partial_{t} \mathcal{E}(E)$ satisfies the identity

$$
\begin{aligned}
\partial_{t} \mathcal{E}(E)= & \sum_{|\alpha| \leq s}\left\langle D^{\alpha} E, A^{0, \varepsilon} D^{\alpha} \partial_{t} E\right\rangle_{L^{2}}=\sum_{|\alpha| \leq s}\left\langle D^{\alpha} E, \sum_{j k} A^{0, \varepsilon} D^{\alpha} \partial_{j}\left(B^{j k}\left(\overline{\mathbf{u}}^{\varepsilon}\right) \partial_{k} E\right)\right\rangle_{L^{2}} \\
& +\sum_{|\alpha| \leq s}\left\langle D^{\alpha} E, \sum_{j k} A^{0, \varepsilon} D^{\alpha} \partial_{j}\left(\partial_{\mathbf{u}} B^{j k}\left(\overline{\mathbf{u}}^{\varepsilon}\right) E \partial_{k} \overline{\mathbf{u}}^{\varepsilon}\right)\right\rangle_{L^{2}}-\sum_{|\alpha| \leq s}\left\langle D^{\alpha} E, \sum_{j} A^{0, \varepsilon} D^{\alpha} \partial_{j}\left(A^{j} E\right)\right\rangle_{L^{2}} \\
& -\sum_{|\alpha| \leq s}\left\langle D^{\alpha} E, Q^{\varepsilon}(\mathbf{v})\right\rangle_{L^{2}} .
\end{aligned}
$$

To estimate the first three terms in the identity above we argue in the same way as in (2.17)-(2.19). The fourth term can be controlled by

$$
\sum_{|\alpha| \leq s}\left\langle D^{\alpha} E,-Q^{\varepsilon}(\mathbf{v})\right\rangle_{L^{2}} \leq \mathcal{O}(1)\left\|E_{2}\right\|_{H^{s+1}}\left(\|\mathbf{v}\|_{H^{s}}+\left\|\mathbf{v}_{2}\right\|_{H^{s+1}}\right)\left(\|\mathbf{v}\|_{H^{s}}+\|\mathbf{v}\|_{H^{s}}^{s}\right) .
$$

Combining all of these estimates together and using the weighted Young's inequality, we have, as long as $\|\mathbf{v}\|_{H^{s}}$ remains sufficiently small, that

$$
\partial_{t} \mathcal{E}(E) \leq-\frac{\theta}{2}\left\|E_{2}\right\|_{H^{s+1}}^{2}+\mathcal{O}(1)\|E\|_{H^{s}}^{2}+\mathcal{O}(1)\|\mathbf{v}\|_{H^{s}}^{2}\left(\|\mathbf{v}\|_{H^{s}}^{2}+\left\|\mathbf{v}_{2}\right\|_{H^{s+1}}^{2}\right) .
$$

Also, since $\|\mathbf{v}(\cdot, t)\|_{H^{s}} \leq C\left\|\mathbf{v}_{0}\right\|_{H^{s}}$, we obtain that

$$
\partial_{t} \mathcal{E}(E) \leq-\frac{\theta}{2}\left\|E_{2}\right\|_{H^{s+1}}^{2}+\mathcal{O}(1)\|E\|_{H^{s}}^{2}+\mathcal{O}(1)\left\|\mathbf{v}_{0}\right\|_{H^{s}}^{2}\left(\|\mathbf{v}\|_{H^{s}}^{2}+\left\|\mathbf{v}_{2}\right\|_{H^{s+1}}^{2}\right)
$$


Using (2.13), Gronwall's inequality and the fact that the error function satisfies the initial condition $E(\cdot, 0)=0$, we conclude that

$$
\mathcal{E}(E(T)) \leq \mathcal{O}(1)\left\|\mathbf{v}_{0}\right\|_{H^{s}}^{2} \int_{0}^{T}\left(\|\mathbf{v}(\cdot, t)\|_{H^{s}}^{2}+\left\|\mathbf{v}_{2}\right\|_{H^{s+1}}^{2}\right) d t \leq \mathcal{O}(1)\left\|\mathbf{v}_{0}\right\|_{H^{s}}^{4}
$$

which implies that $\|E(\cdot, T)\|_{H^{s}} \leq \mathcal{O}(1)\left\|\mathbf{v}_{0}\right\|_{H^{s}}^{2}$, proving the lemma.

\section{O(2) BIFURCATION FOR THE GENERAL CASE}

In this section we prove various preliminary results needed to prove our main result of this paper, the existence of $O(2)$-Hopf bifurcation under a spectral criterion (Hypothesis $D_{\varepsilon}$ ) described in detail in the introduction. More precisely, we are looking to prove the existence of periodic solution of equation (2.2) (subject to the constraint $m \mathbf{v}=0$ ), of period $T>0$, by solving for $T$ as a function of the initial data $\mathbf{v}(0)$ and the bifurcation parameter $\varepsilon$ in the fixed point equation associated to the return map of $(2.2)(\bmod m)$. Furthermore, we reduce this infinite dimensional nonlinear system to a finite dimensional system by using the a special variant of the Lyapunov-Schmidt reduction method, introduced in [TZ2] and refined in [TZ3].

In what follows we are going to consider the operator $L(\varepsilon)$ defined in (1.4) as a second order differential operator from $H^{2}\left(\mathbb{R} \times[-\pi, \pi], \mathbb{C}^{n}\right)$ to $L^{2}\left(\mathbb{R} \times[-\pi, \pi], \mathbb{C}^{n}\right)$. By taking Fourier Transform in $x_{2} \in[-\pi, \pi]$, we can identify $L^{2}\left(\mathbb{R} \times[-\pi, \pi], \mathbb{C}^{n}\right)$ with $\ell^{2}\left(\mathbb{Z}, L^{2}\left(\mathbb{R}, \mathbb{C}^{n}\right)\right)$ and $H^{p}\left(\mathbb{R} \times[-\pi, \pi], \mathbb{C}^{n}\right)$ with $\bigoplus_{k \in \mathbb{Z}} \hat{H}_{k}^{p}\left(\mathbb{R}, \mathbb{C}^{n}\right), p=1,2$, where the Hilbert space $\hat{H}_{k}^{p}\left(\mathbb{R}, \mathbb{C}^{n}\right)$ is the Sobolev space $H^{p}\left(\mathbb{R}, \mathbb{C}^{n}\right)$, $p=1,2$, with the scalar products

$$
\begin{gathered}
\langle f, g\rangle_{\hat{H}_{k}^{1}\left(\mathbb{R}, \mathbb{C}^{n}\right)}=\left(1+k^{2}\right)\langle f, g\rangle_{L^{2}}+\left\langle\partial_{x_{1}} f, \partial_{x_{1}} g\right\rangle_{L^{2}} \\
\langle f, g\rangle_{\hat{H}_{k}^{2}\left(\mathbb{R}, \mathbb{C}^{n}\right)}=\left(1+k^{2}+k^{4}\right)\langle f, g\rangle_{L^{2}}+\left(1+2 k^{2}\right)\left\langle\partial_{x_{1}} f, \partial_{x_{1}} g\right\rangle_{L^{2}}+\left\langle\partial_{x_{1}}^{2} f, \partial_{x_{1}}^{2} g\right\rangle_{L^{2}} .
\end{gathered}
$$

The operator $L(\varepsilon)$ can be identified with $\left(L_{k}(\varepsilon)\right)_{k \in \mathbb{Z}}$, where $L_{k}(\varepsilon): \hat{H}_{k}^{2}\left(\mathbb{R}, \mathbb{C}^{n}\right) \rightarrow L^{2}\left(\mathbb{R}, \mathbb{C}^{n}\right)$ are defined by $L_{k}(\varepsilon)=\widehat{L(\varepsilon)}(k)$. A simple computation shows that

$$
L_{k}(\varepsilon)=L_{0}(\varepsilon)+\mathrm{i} k J(\varepsilon)-k^{2} \bar{B}^{22}\left(\varepsilon, x_{1}\right),
$$

where

$$
L_{0}(\varepsilon)=\partial_{x_{1}}\left[\bar{B}^{11}\left(\varepsilon, x_{1}\right) \partial_{x_{1}}-\bar{A}^{1}\left(\varepsilon, x_{1}\right)\right]
$$

and $J(\varepsilon)$ is the first order operator defined by

$$
J(\varepsilon)=\left[\bar{B}^{12}\left(\varepsilon, x_{1}\right)+\bar{B}^{21}\left(\varepsilon, x_{1}\right)\right] \partial_{x_{1}}+\bar{B}\left(\varepsilon, x_{1}\right)-\bar{A}^{2}\left(\varepsilon, x_{1}\right) .
$$

Here the functions $\bar{A}^{j}, \bar{B}^{j k}:(-\delta, \delta) \times \mathbb{R} \rightarrow \mathbb{R}^{n \times n}$ are defined by composing the functions $A^{j}$ and $B^{j k}$, respectively, with $\left(\varepsilon, \overline{\mathbf{u}}^{\varepsilon}\left(x_{1}\right)\right)$.

Remark 3.1. Since the operator $L_{k}(\varepsilon), k \in \mathbb{Z}$, are one-dimensional differential operators ((3.3), (3.4)), we note that its eigenvalues can be obtained, away from the essential spectrum, as zeros of the classical Evans function, denoted $D(\cdot, k, \varepsilon)$, see, e.g [Z1, Z2]. At the special eigenvalues $\lambda=0$ of $L_{0}(\varepsilon)$, which are embedded in essential spectra, the zeros of the Evans function carry additional information determining asymptotic stability [Z1].

The next step in constructing the $O(2)$ bifurcation is to construct the time- $T$ evolution map of the equation $(2.2)(\bmod m)$. An crucial role in this construction is played by the rotational invariance and by the $x_{2} \rightarrow-x_{2}$ invariance of this equation. More precisely, we note that there 
exists a non-degenerate rotation group of linear operators $\{R(\theta)\}_{\theta \in \mathbb{R}}$ on $L^{2}\left(\mathbb{R} \times[-\pi, \pi], \mathbb{C}^{n}\right)$, with $R(\theta)^{*}=R(-\theta)$, for all $\theta \in \mathbb{R}^{8}$, and a bounded symmetry $S$ on $L^{2}\left(\mathbb{R} \times[-\pi, \pi], \mathbb{R}^{n}\right)$ satisfying

$$
\mathcal{F}(\varepsilon ; R(\theta) \mathbf{u})=R(\theta) \mathcal{F}(\varepsilon ; \mathbf{u}), \quad \mathcal{F}(\varepsilon ; S \mathbf{u})=S \mathcal{F}(\varepsilon ; \mathbf{u}),
$$

for all $\mathbf{u} \in H^{2}\left(\mathbb{R} \times[-\pi, \pi], \mathbb{R}^{n}\right), \theta \in \mathbb{R}$ and $\varepsilon \in(-\delta, \delta)$. The group $\left\{R(\theta\}_{\theta \in \mathbb{R}}\right.$ and $S$ satisfy the following condition

$$
R(\theta) S=S R(-\theta) \quad \text { for all } \quad \theta \in \mathbb{R} .
$$

Since $L(\varepsilon)=\frac{\partial \mathcal{F}}{\partial \mathbf{u}}\left(\varepsilon ; \overline{\mathbf{u}}^{\varepsilon}\right)$, from $(3.6)$ we obtain that $H^{2}\left(\mathbb{R} \times[-\pi, \pi], \mathbb{C}^{n}\right)=\operatorname{dom}(L(\varepsilon))$ is invariant under $S$ and $R(\theta)$, for all $\theta \in \mathbb{R}$ and

$$
L(\varepsilon) R(\theta)=R(\theta) L(\varepsilon), \quad L(\varepsilon) S=S L(\varepsilon) \text { for all } \theta \in \mathbb{R}, \varepsilon \in(-\delta, \delta) .
$$

We introduce $G$ the generator of the rotation group $\{R(\theta)\}_{\theta \in \mathbb{R}}$ and we note that $\operatorname{dom}(L(\varepsilon)) \subset$ $\operatorname{dom}(G)$ for all $\varepsilon \in(-\delta, \delta)$. Moreover, from (3.7) and (3.8) and since $\{R(\theta)\}_{\theta \in \mathbb{R}}$ is a rotation group, we infer that

$$
G^{*}=-G, \quad G L(\varepsilon)=L(\varepsilon) G, \quad G S=-S G \text { for all } \varepsilon \in(-\delta, \delta) .
$$

Very important to our reduction are the eigenspaces $\Sigma_{ \pm}(\varepsilon)$ associated to the eigenvalues $\lambda_{ \pm}(\varepsilon)$ of $L(\varepsilon)$. In the next lemma we summarize a few basic properties of these eigenspaces.

Lemma 3.2. Assume Hypotheses (A1)-(A3), (B1), (H0)-(H6) and $\left(D_{\varepsilon}\right)$. For any $\varepsilon \in(-\delta, \delta)$ the following assertions hold true:

(i) The subspaces $\Sigma_{ \pm}(\varepsilon)$ are invariant under $G, S$ and $R(\theta)$ for any $\theta \in \mathbb{R}$;

(ii) There exits $\alpha(\varepsilon) \in \mathbb{R} \backslash\{0\}$ such that $\sigma\left(G_{\mid \Sigma_{+}(\varepsilon)}\right)=\{ \pm \mathrm{i} \alpha(\varepsilon)\}$;

(iii) Let $\mathbf{w}^{\varepsilon} \in \Sigma_{+}(\varepsilon)$ be the eigenfunction (unique up to a scalar multiple) of $G_{\mid \Sigma_{+}(\varepsilon)}$ corresponding to the eigenvalue $\mathrm{i} \alpha(\varepsilon)$. Then, the eigenspaces $\Sigma_{ \pm}(\varepsilon)$ can be represented as follows:

$$
\Sigma_{+}(\varepsilon)=\operatorname{Sp}\left\{\mathbf{w}^{\varepsilon}, S \mathbf{w}^{\varepsilon}\right\}, \quad \Sigma_{-}(\varepsilon)=\operatorname{Sp}\left\{\overline{\mathbf{w}^{\varepsilon}}, S \overline{\mathbf{w}^{\varepsilon}}\right\} .
$$

Proof. Assertion (i) follows from the fact that the operator $L(\varepsilon)$ commutes with $R(\theta), S$ and $G$ by (3.8) and (3.9).

(ii) From (3.9) we have that $G^{*}=-G$ and since by (i) $\Sigma_{+}(\varepsilon)$ is invariant under $G$, we infer that $\left(G_{\mid \Sigma_{+}(\varepsilon)}\right)^{*}=-G_{\mid \Sigma_{+}(\varepsilon)}$. It follows that $\sigma\left(G_{\mid \Sigma_{+}(\varepsilon)}\right) \subset \mathrm{i} \mathbb{R}$. Since $\operatorname{dim}\left(\Sigma_{+}(\varepsilon)\right)=2$, we conclude that there exists $\alpha(\varepsilon) \in \mathbb{R} \backslash\{0\}$ such that $\sigma\left(G_{\mid \Sigma_{+}(\varepsilon)}\right)=\{ \pm \mathrm{i} \alpha(\varepsilon)\}$. Taking into account that the group of rotations $\{R(\theta)\}$ is non-degenerate, we infer that $\operatorname{ker} G=\{0\}$, which implies that $\alpha(\varepsilon) \neq 0$, proving (ii).

(iii) We note that since $L(\varepsilon) \mathbf{w}^{\varepsilon}=\lambda_{+}(\varepsilon) \mathbf{w}^{\varepsilon}$, from (3.8) it follows that

$$
L(\varepsilon) S \mathbf{w}^{\varepsilon}=S L(\varepsilon) \mathbf{w}^{\varepsilon}=\lambda_{+}(\varepsilon) S \mathbf{w}^{\varepsilon},
$$

and thus $S \mathbf{w}^{\varepsilon} \in \Sigma_{+}(\varepsilon)$. Next, we will show that $\mathbf{w}^{\varepsilon}$ and $S \mathbf{w}^{\varepsilon}$ are linearly independent. From (3.9) we obtain that $G\left(S \mathbf{w}^{\varepsilon}\right)=-S G \mathbf{w}^{\varepsilon}=-\mathrm{i} \alpha(\varepsilon) S \mathbf{w}^{\varepsilon}$. Thus, $\mathbf{w}^{\varepsilon}$ and $S \mathbf{w}^{\varepsilon}$ are eigenfunctions of the same operator corresponding to different eigenvalues, which proves that $\mathbf{w}^{\varepsilon}$ and $S \mathbf{w}^{\varepsilon}$ are linearly independent. Using again that $\operatorname{dim}\left(\Sigma_{+}(\varepsilon)\right)=2$ we have that $\Sigma_{+}(\varepsilon)=\operatorname{Sp}\left\{\mathbf{w}^{\varepsilon}, S \mathbf{w}^{\varepsilon}\right\}$. Since $\overline{L(\varepsilon) \mathbf{u}}=L(\varepsilon) \overline{\mathbf{u}}$ for any $\mathbf{u} \in H^{2}\left(\mathbb{R} \times[-\pi, \pi], \mathbb{C}^{n}\right)$ we readily infer that $\Sigma_{-}(\varepsilon)=\operatorname{Sp}\left\{\overline{\mathbf{w}^{\varepsilon}}, S \overline{\mathbf{w}^{\varepsilon}}\right\}$.

Since $\lambda_{ \pm}(\varepsilon)$ is an eigenvalue of $L(\varepsilon)$ and $\overline{\lambda_{+}(\varepsilon)}=\lambda_{-}(\varepsilon)$ for any $\varepsilon \in(-\delta, \delta)$, we know that $\lambda_{ \pm}(\varepsilon)$ are also eigenvalues of $L(\varepsilon)^{*}$ for any $\varepsilon \in(-\delta, \delta)$. Moreover, if we denote by $\tilde{\Sigma}_{ \pm}(\varepsilon)$ the eigenspaces associated to eigenvalues $\lambda_{ \pm}(\varepsilon)$ of $L(\varepsilon)^{*}$, we have that $\operatorname{dim}\left(\tilde{\Sigma}_{ \pm}(\varepsilon)\right)=2$ for any $\varepsilon \in(-\delta, \delta)$. The properties satisfied by the eigenspaces $\tilde{\Sigma}_{ \pm}(\varepsilon)$ are similar to the ones described in Lemma 3.2 as shown in the lemma below.

\footnotetext{
${ }^{8}$ We can naturally extend the operator $S$ to the complexification of its domain such that $S^{*}=S$
} 
Lemma 3.3. Assume Hypotheses (A1)-(A3), (B1), (H0)-(H6) and $\left(D_{\varepsilon}\right)$. For any $\varepsilon \in(-\delta, \delta)$ the following assertions hold true:

(i) The subspaces $\tilde{\Sigma}_{ \pm}(\varepsilon)$ are invariant under $G, S$ and $R(\theta)$ for any $\theta \in \mathbb{R}$;

(ii) $\sigma\left(G_{\mid \tilde{\Sigma}_{-}(\varepsilon)}\right)=\{ \pm \mathrm{i} \alpha(\varepsilon)\}$. The function $\alpha(\varepsilon)$ is the one introduced in Lemma 3.2(ii);

(iii) Let $\tilde{\mathbf{w}}^{\varepsilon} \in \Sigma_{-}(\varepsilon)$ be the eigenfunction (unique up to a scalar multiple) of $G_{\mid \tilde{\Sigma}_{+}(\varepsilon)}$ corresponding to the eigenvalue $\mathrm{i} \alpha(\varepsilon)$. Without loss of generality we can choose $\tilde{\mathbf{w}}^{\varepsilon}$ such that $\left\langle\mathbf{w}^{\varepsilon}, \tilde{\mathbf{w}}^{\varepsilon}\right\rangle_{L^{2}}=1$. Then, the eigenspaces $\tilde{\Sigma}_{ \pm}(\varepsilon)$ can be represented as follows:

$$
\tilde{\Sigma}_{-}(\varepsilon)=\operatorname{Sp}\left\{\tilde{\mathbf{w}}^{\varepsilon}, S \tilde{\mathbf{w}}^{\varepsilon}\right\}, \quad \tilde{\Sigma}_{+}(\varepsilon)=\operatorname{Sp}\left\{\overline{\tilde{\mathbf{w}}^{\varepsilon}}, S \overline{\tilde{\mathbf{w}}^{\varepsilon}}\right\} .
$$

Proof. First, we note that by taking adjoint in (3.8) and (3.9) we obtain that the operator $L(\varepsilon)^{*}$ commutes with $R(\theta), S$ and $G$ for any $\theta \in \mathbb{R}$ and $\varepsilon \in(-\delta, \delta)$. Since, in addition $\operatorname{dim}\left(\tilde{\Sigma}_{ \pm}(\varepsilon)\right)=2$ for any $\varepsilon \in(-\delta, \delta)$, we can obtain all properties above by using the same arguments we used in Lemma 3.2. The only thing left to prove is that the operators $G_{\mid \Sigma_{+}(\varepsilon)}$ and $G_{\mid \tilde{\Sigma}_{+}(\varepsilon)}$ have the same eigenvalues. Since $G^{*}=-G$ and the eigenspace $\tilde{\Sigma}_{+}(\varepsilon)$ is invariant under $G$ we have that $\left(G_{\mid \tilde{\Sigma}_{+}(\varepsilon)}\right)^{*}=-G_{\mid \tilde{\Sigma}_{+}(\varepsilon)}$, and thus $\sigma\left(G_{\mid \tilde{\Sigma}_{+}(\varepsilon)}\right)=\{ \pm \mathrm{i} \beta(\varepsilon)\}$ for some $\beta(\varepsilon) \in \mathbb{R}$. To finish the proof all we need to do is to show that $|\alpha(\varepsilon)|=|\beta(\varepsilon)|$. Indeed, one can readily check that

$$
\mathrm{i} \alpha(\varepsilon)\left\langle\mathbf{w}^{\varepsilon}, \tilde{\mathbf{w}}^{\varepsilon}\right\rangle_{L^{2}}=\left\langle G \mathbf{w}^{\varepsilon}, \tilde{\mathbf{w}}^{\varepsilon}\right\rangle_{L^{2}}=\left\langle\mathbf{w}^{\varepsilon}, G^{*} \tilde{\mathbf{w}}^{\varepsilon}\right\rangle_{L^{2}}=\left\langle\mathbf{w}^{\varepsilon},-G \tilde{\mathbf{w}}^{\varepsilon}\right\rangle_{L^{2}}=-\mathrm{i} \beta(\varepsilon)\left\langle\mathbf{w}^{\varepsilon}, \tilde{\mathbf{w}}^{\varepsilon}\right\rangle_{L^{2}},
$$

finishing the proof.

Lemma 3.4. Assume Hypotheses (A1)-(A3), (B1), (H0)-(H6) and $\left(D_{\varepsilon}\right)$. For any $\varepsilon \in(-\delta, \delta)$ the following assertion holds true:

$$
\left\langle S \mathbf{w}^{\varepsilon}, \tilde{\mathbf{w}}^{\varepsilon}\right\rangle_{L^{2}}=0
$$

Proof. To prove (3.11) we use (3.9), Lemma 3.2 and Lemma 3.3 as follows: first we compute

$$
\left\langle S G \mathbf{w}^{\varepsilon}, \tilde{\mathbf{w}}^{\varepsilon}\right\rangle_{L^{2}}=\left\langle S\left(\mathrm{i} \alpha(\varepsilon) \mathbf{w}^{\varepsilon}\right), \tilde{\mathbf{w}}^{\varepsilon}\right\rangle_{L^{2}}=\mathrm{i} \alpha(\varepsilon)\left\langle S \mathbf{w}^{\varepsilon}, \tilde{\mathbf{w}}^{\varepsilon}\right\rangle_{L^{2}} .
$$

In addition,

$$
\begin{aligned}
\left\langle S G \mathbf{w}^{\varepsilon}, \tilde{\mathbf{w}}^{\varepsilon}\right\rangle_{L^{2}} & =-\left\langle G S \mathbf{w}^{\varepsilon}, \tilde{\mathbf{w}}^{\varepsilon}\right\rangle_{L^{2}}=-\left\langle S \mathbf{w}^{\varepsilon}, G^{*} \tilde{\mathbf{w}}^{\varepsilon}\right\rangle_{L^{2}}=-\left\langle S \mathbf{w}^{\varepsilon},-G \tilde{\mathbf{w}}^{\varepsilon}\right\rangle_{L^{2}} \\
& =\left\langle S \mathbf{w}^{\varepsilon}, G \tilde{\mathbf{w}}^{\varepsilon}\right\rangle_{L^{2}}=\left\langle S \mathbf{w}^{\varepsilon}, \mathrm{i} \alpha(\varepsilon) \tilde{\mathbf{w}}^{\varepsilon}\right\rangle_{L^{2}}=-\mathrm{i} \alpha(\varepsilon)\left\langle S \mathbf{w}^{\varepsilon}, \tilde{\mathbf{w}}^{\varepsilon}\right\rangle_{L^{2}} .
\end{aligned}
$$

Using the group property of $\{R(\theta)\}_{\theta \in \mathbb{R}}$, one readily infers (3.12) from Lemma 3.2 and Lemma 3.3.

In the next lemma we describe the relation between the eigenspaces $\Sigma_{ \pm}(\varepsilon)$ and the kernel of $m$.

Lemma 3.5. Assume Hypotheses (A1)-(A3), (B1), (HO)-(H6) and $\left(D_{\varepsilon}\right)$. Then, the following inclusion holds true:

$$
\Sigma_{ \pm}(\varepsilon) \subset \operatorname{ker} m \quad \text { for all } \varepsilon \in(-\delta, \delta) .
$$

Proof. Let $\mathbf{v}$ be a vector contained in $\Sigma_{+}(\varepsilon)$ or $\Sigma_{-}(\varepsilon)$ and $\lambda=\lambda_{+}(\varepsilon)$ or $\left.\lambda=\lambda_{-}(\varepsilon)\right\}$, respectively. Since $\Sigma_{ \pm}(\varepsilon)$ are eigenspaces of $L(\varepsilon)$ we obtain that $L(\varepsilon) \mathbf{v}=\lambda \mathbf{v}$. Using (1.5) we obtain that

$$
(\Gamma(\varepsilon)-\lambda) m \mathbf{v}=m L(\varepsilon) \mathbf{v}-\lambda m \mathbf{v}=0 .
$$

From Hypothesis $\left(D_{\varepsilon}\right)$ (ii) and $(\mathrm{v})$ we infer that $\lambda$ is not an eigenvalue of $\Gamma(\varepsilon)$, which implies that $m \mathbf{v}=0$, proving the lemma. 
Throughout this paper we denote by $\Pi_{ \pm}(\varepsilon)$ the orthogonal projection onto $\Sigma_{ \pm}(\varepsilon)$ parallel to $\tilde{\Sigma}_{ \pm}(\varepsilon)^{\perp}$ and by $\Pi(\varepsilon)=I-\Pi_{+}(\varepsilon)-\Pi_{-}(\varepsilon)$. Also, we introduce $\Sigma(\varepsilon):=\operatorname{Range}(\Pi(\varepsilon))$ the complement of $\Sigma_{+}(\varepsilon) \oplus \Sigma_{-}(\varepsilon)$. From (3.10) and (3.11) we know that the projectors $\Pi_{ \pm}(\varepsilon)$ have the following representation:

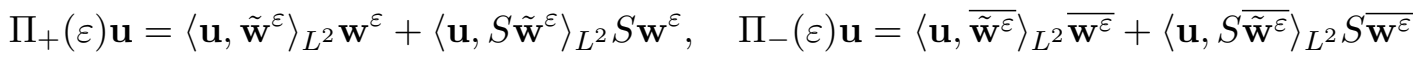

for any $\mathbf{u} \in L^{2}\left(\mathbb{R} \times[-\pi, \pi], \mathbb{C}^{n}\right)$. Using the fact that $\mathbf{w}^{\varepsilon}$ and $S \mathbf{w}^{\varepsilon}$ are eigenfunctions of $L(\varepsilon)$ associated to the eigenvalue $\lambda_{+}(\varepsilon)$ and $\tilde{\mathbf{w}}^{\varepsilon}$ and $S \tilde{\mathbf{w}}^{\varepsilon}$ are eigenvalues of $L(\varepsilon)^{*}$ associated to the eigenvalue $\lambda_{-}(\varepsilon)=\overline{\lambda_{+}(\varepsilon)}$ for any $\varepsilon \in(-\delta, \delta)$, one can readily check that

$$
\Pi_{ \pm}(\varepsilon) L(\varepsilon)=L(\varepsilon) \Pi_{ \pm}(\varepsilon), \quad \Pi(\varepsilon) L(\varepsilon)=L(\varepsilon) \Pi(\varepsilon) \quad \text { for any } \quad \varepsilon \in(-\delta, \delta) .
$$

Next, we note that equation (2.2) is of the form

$$
\mathbf{v}^{\prime}(t)=L(\varepsilon) \mathbf{v}(t)+\mathcal{N}(\varepsilon ; \mathbf{v}(t)), \quad t \geq 0
$$

where the non-linear function $\mathcal{N}:(-\delta, \delta) \times H^{2}\left(\mathbb{R} \times[-\pi, \pi], \mathbb{C}^{n}\right) \rightarrow L^{2}\left(\mathbb{R} \times[-\pi, \pi], \mathbb{C}^{n}\right)$ is defined by

$$
\mathcal{N}(\varepsilon ; \mathbf{u})=\mathcal{F}(\varepsilon ; \mathbf{u})-L(\varepsilon) \mathbf{u} .
$$

From (3.6) and (3.8) we conclude that

$$
\mathcal{N}(\varepsilon ; R(\theta) \mathbf{u})=R(\theta) \mathcal{N}(\varepsilon ; \mathbf{u}), \quad \mathcal{N}(\varepsilon ; S \mathbf{u})=S \mathcal{N}(\varepsilon ; \mathbf{u}),
$$

for all $\mathbf{u} \in H^{2}\left(\mathbb{R} \times[-\pi, \pi], \mathbb{R}^{n}\right), \theta \in \mathbb{R}$ and $\varepsilon \in(-\delta, \delta)$. To construct the return map of $(2.2)$ $(\bmod m)$, we start by coordinatizing equation (3.16) as follows:

$$
\mathbf{v}_{ \pm}(t)=\Pi_{ \pm}(\varepsilon) \mathbf{v}(t), \quad \tilde{\mathbf{v}}(t)=\Pi(\varepsilon) \mathbf{v}(t), \quad t \geq 0, \varepsilon \in(-\delta, \delta) .
$$

We introduce the functions $\phi, \psi: \mathbb{R}_{+} \rightarrow \mathbb{C}$ by $\phi(t)=\left\langle\mathbf{v}(t), \tilde{\mathbf{w}}^{\varepsilon}\right\rangle_{L^{2}}$ and $\psi(t)=\left\langle\mathbf{v}(t), S \tilde{\mathbf{w}}^{\varepsilon}\right\rangle_{L^{2}}$. Since we are looking for real-valued solutions of our PDE-system we are interested in finding solution $\mathbf{v}$ of equation (3.16) satisfying $\mathbf{v}(t)=\overline{\mathbf{v}(t)}$ for each $t \geq 0$. It follows that

$$
\overline{\phi(t)}=\left\langle\mathbf{v}(t), \overline{\tilde{\mathbf{w}}^{\varepsilon}}\right\rangle_{L^{2}}, \quad \overline{\psi(t)}=\left\langle\mathbf{v}(t), S \overline{\tilde{\mathbf{w}}^{\varepsilon}}\right\rangle_{L^{2}} \quad \text { for all } \quad t \geq 0,
$$

which implies that

$$
\mathbf{v}_{-}(t)=\overline{\mathbf{v}_{+}(t)} \text { for all } t \geq 0 .
$$

Next, we rewrite the system (3.16) in the new variables $(\phi, \psi, \tilde{\mathbf{v}})$. From Lemma 3.3(iii) and Lemma 3.4 we conclude that (3.16), subject to the constraint $m \mathbf{v}=0$, is equivalent to the system

$$
\left\{\begin{array}{l}
\phi^{\prime}=\lambda_{+}(\varepsilon) \phi+\left\langle\mathcal{N}\left(\varepsilon ; \phi \mathbf{w}^{\varepsilon}+\psi S \mathbf{w}^{\varepsilon}+\tilde{\mathbf{v}}\right), \tilde{\mathbf{w}}^{\varepsilon}\right\rangle_{L^{2}} \\
\psi^{\prime}=\lambda_{+}(\varepsilon) \psi+\left\langle\mathcal{N}\left(\varepsilon ; \phi \mathbf{w}^{\varepsilon}+\psi S \mathbf{w}^{\varepsilon}+\tilde{\mathbf{v}}\right), S \tilde{\mathbf{w}}^{\varepsilon}\right\rangle_{L^{2}} \\
\tilde{\mathbf{v}}^{\prime}=L^{\Pi}(\varepsilon) \tilde{\mathbf{v}}+\Pi(\varepsilon) \mathcal{N}\left(\varepsilon ; \phi \mathbf{w}^{\varepsilon}+\psi S \mathbf{w}^{\varepsilon}+\tilde{\mathbf{v}}\right)
\end{array}\right.
$$

where $L^{\Pi}(\varepsilon)=L(\varepsilon)_{\mid \operatorname{Range}(\Pi(\varepsilon))}$.

Since the linear operator $L(\varepsilon)$ generates a $C_{0}$-semigroup, (see, e.g., [Lun] or [Z2]), from (3.15) we infer that $L^{\Pi}(\varepsilon)$ generates a $C_{0}$-semigroup. Next, we integrate $(3.22)$ in $t \in[0, T]$ using the variation of constants formula to obtain the system

$$
\left\{\begin{array}{l}
\phi(T)=e^{T \lambda_{+}(\varepsilon)} \phi(0)+\int_{0}^{T} e^{(T-t) \lambda_{+}(\varepsilon)} \Phi(\phi(s), \psi(s), \tilde{\mathbf{v}}(s), \varepsilon) d s \\
\psi(T)=e^{T \lambda_{+}(\varepsilon)} \psi(0)+\int_{0}^{T} e^{(T-t) \lambda_{+}(\varepsilon)} \Psi(\phi(s), \psi(s), \tilde{\mathbf{v}}(s), \varepsilon) d s \\
\tilde{\mathbf{v}}(T)=e^{T L^{\Pi}(\varepsilon)} \Pi(\varepsilon) \tilde{\mathbf{v}}(0)+\int_{0}^{T} e^{(T-t) L^{\Pi}(\varepsilon)} \tilde{\mathcal{V}}(\phi(s), \psi(s), \tilde{\mathbf{v}}(s), \varepsilon) d s
\end{array} .\right.
$$

Here we denoted by $\left\{e^{t L^{\Pi}(\varepsilon)}\right\}_{t \geq 0}$ the $C_{0}$-semigroup generated by the operator $L^{\Pi}(\varepsilon)$. The nonlinearities $\Phi, \Psi: \mathbb{C}^{2} \times H^{2}\left(\mathbb{R} \times[-\pi, \pi], \mathbb{C}^{n}\right) \times(-\delta, \delta) \rightarrow \mathbb{C}$, are defined by

$$
\Phi\left(z_{1}, z_{2}, \tilde{\mathbf{v}}, \varepsilon\right)=\left\langle\mathcal{N}\left(\varepsilon ; z_{1} \mathbf{w}^{\varepsilon}+z_{2} S \mathbf{w}^{\varepsilon}+\tilde{\mathbf{v}}\right), \tilde{\mathbf{w}}^{\varepsilon}\right\rangle_{L^{2}}, \Psi\left(z_{1}, z_{2}, \tilde{\mathbf{v}}, \varepsilon\right)=\left\langle\mathcal{N}\left(\varepsilon ; z_{1} \mathbf{w}^{\varepsilon}+z_{2} S \mathbf{w}^{\varepsilon}+\tilde{\mathbf{v}}\right), S \tilde{\mathbf{w}}^{\varepsilon}\right\rangle_{L^{2}} .
$$


In addition, the nonlinear map $\tilde{\mathcal{V}}: \mathbb{C}^{2} \times H^{2}\left(\mathbb{R} \times[-\pi, \pi], \mathbb{C}^{n}\right) \times(-\delta, \delta) \rightarrow L^{2}\left(\mathbb{R} \times[-\pi, \pi], \mathbb{C}^{n}\right)$ is defined by

$$
\tilde{\mathcal{V}}\left(z_{1}, z_{2}, \tilde{\mathbf{v}}, \varepsilon\right)=\Pi(\varepsilon) \mathcal{N}\left(\varepsilon ; z_{1} \mathbf{w}^{\varepsilon}+z_{2} S \mathbf{w}^{\varepsilon}+\tilde{\mathbf{v}}\right)
$$

To prove existence of periodic solutions of period $T$ of $(2.2)(\bmod m)$, it is enough to show that we can solve for $T$ in the $T$-return map of (3.23) in terms of the initial conditions. This is equivalent with finding fixed points of the period map defined by (3.23) or with finding zeros of the displacement map Disp $=\left(\mathbf{D i s p}_{1}, \mathbf{D i s p}_{2}, \mathbf{D i s p}_{3}\right): \mathbb{C}^{2} \times H^{2}\left(\mathbb{R} \times[-\pi, \pi], \mathbb{C}^{n}\right) \times(-\delta, \delta) \times(0, \infty) \rightarrow$ $\mathbb{C}^{2} \times L^{2}\left(\mathbb{R} \times[-\pi, \pi], \mathbb{C}^{n}\right)$ defined by

$$
\begin{aligned}
& \operatorname{Disp}_{1}\left(a_{1}, a_{2}, \tilde{\mathbf{v}}_{0}, \varepsilon, T\right)=\left(e^{T \lambda_{+}(\varepsilon)}-1\right) a_{1}+\int_{0}^{T} e^{(T-t) \lambda_{+}(\varepsilon)} \Phi(\phi(s), \psi(s), \tilde{\mathbf{v}}(s), \varepsilon) d s ; \\
& \operatorname{Disp}_{2}\left(a_{1}, a_{2}, \tilde{\mathbf{v}}_{0}, \varepsilon, T\right)=\left(e^{T \lambda_{+}(\varepsilon)}-1\right) a_{2}+\int_{0}^{T} e^{(T-t) \lambda_{+}(\varepsilon)} \Psi(\phi(s), \psi(s), \tilde{\mathbf{v}}(s), \varepsilon) d s ;
\end{aligned}
$$

$$
\operatorname{Disp}_{3}\left(a_{1}, a_{2}, \tilde{\mathbf{v}}_{0}, \varepsilon, T\right)=\left(e^{T L^{\Pi}(\varepsilon)} \Pi(\varepsilon)-I\right) \tilde{\mathbf{v}}_{0}+\int_{0}^{T} e^{(T-t) L^{\Pi}(\varepsilon)} \tilde{\mathcal{V}}(\phi(s), \psi(s), \tilde{\mathbf{v}}(s), \varepsilon) d s,
$$

satisfying the condition $\tilde{\mathbf{v}}_{0} \in \operatorname{Range}(\Pi(\varepsilon))$, where $(\phi, \psi, \tilde{\mathbf{v}})$ is a solution of (3.22) with initial condition $(\phi, \psi, \tilde{\mathbf{v}})(0)=\left(a_{1}, a_{2}, \tilde{\mathbf{v}}_{0}\right)$. At this moment it is crucial to eliminate $\tilde{\mathbf{v}}_{0}$ from the system

$$
\operatorname{Disp}\left(a_{1}, a_{2}, \tilde{\mathbf{v}}_{0}, \varepsilon, T\right)=0
$$

using a special form of the Lyapunov-Schmidt reduction in order to obtain a finite dimensional system. To apply the Lyapunov-Schmidt reduction method, following [TZ1]-[TZ4], we need to investigate some of the properties of $L^{\Pi}(\varepsilon)=L(\varepsilon)_{\mid \operatorname{Range}(\Pi(\varepsilon))}$. More precisely, we need to investigate the (right) invertibility of $e^{T L^{\Pi}(\varepsilon)}-I$ for $T>0$ to be chosen later. From Hypothesis $\left(D_{\varepsilon}\right)$ we infer that $L^{\Pi}(\varepsilon)$ has only finitely many eigenvalues with positive real part of finite multiplicity. We define

$$
\theta(\varepsilon)=\min \left\{\operatorname{Re} \lambda>0: \lambda \in \sigma_{\text {point }}(L(\varepsilon))\right\},
$$

and we introduce the spectral projectors

$$
\Pi^{\text {pos }}(\varepsilon)=\text { spectral projection of } \sigma\left(L^{\Pi}(\varepsilon)\right) \cap\{\lambda \in \mathbb{C}: \operatorname{Re} \lambda>\theta(\varepsilon)\}, \quad \Pi^{\text {neg }}(\varepsilon)=\Pi(\varepsilon)-\Pi^{\text {pos }}(\varepsilon) \text {. }
$$

Moreover, if we define $L^{\text {pos }}(\varepsilon)=L(\varepsilon)_{\mid \operatorname{Range}\left(\Pi^{\operatorname{pos}}(\varepsilon)\right)}$ and $L^{\text {neg }}(\varepsilon)=L(\varepsilon)_{\mid \operatorname{Range}\left(\Pi^{\text {neg }}(\varepsilon)\right)}$, from the invariance of the spectral projectors we obtain the following diagonal decomposition on Range $(\Pi(\varepsilon))=$ Range $\left(\Pi^{\text {pos }}(\varepsilon)\right) \oplus \operatorname{Range}\left(\Pi^{\text {neg }}(\varepsilon)\right)$ :

$$
L^{\Pi}(\varepsilon)=\left(\begin{array}{cc}
L^{\operatorname{pos}}(\varepsilon) & 0 \\
0 & L^{\mathrm{neg}}(\varepsilon)
\end{array}\right) .
$$

Since Range $\left(\Pi^{\text {pos }}(\varepsilon)\right)$ is finite dimensional and $\sigma\left(L^{\operatorname{pos}}(\varepsilon)\right) \subset\{\lambda \in \mathbb{C}: \operatorname{Re} \lambda>0\}$, we infer

$$
e^{T L^{\text {pos }}(\varepsilon)}-I \text { is invertible on Range }\left(\Pi^{\text {pos }}(\varepsilon)\right) \text { for all } T>0, \varepsilon \in(-\delta, \delta) .
$$

Taking again Fourier Transform in $x_{2} \in[-\pi, \pi]$, we can identify $L^{\text {neg }}(\varepsilon)$ with $\left(L_{k}^{\text {neg }}(\varepsilon)\right)_{k \in \mathbb{Z}}$ on $\bigoplus_{k \in \mathbb{Z}} \hat{H}_{k}^{2}\left(\mathbb{R}, \mathbb{C}^{n}\right) \cap \mathfrak{f}_{k}\left(\right.$ Range $\left.\left(\Pi^{\text {neg }}(\varepsilon)\right)\right)$. Here $L_{k}^{\text {neg }}(\varepsilon)=\widehat{L^{\text {neg }}(\varepsilon)}(k)$ and $\mathfrak{f}_{k} \mathbf{u}=\hat{\mathbf{u}}(k)$. In addition, using that Range $\left(\Pi^{\text {pos }}(\varepsilon)\right)$ and $\Sigma_{ \pm}(\varepsilon)$ are finite dimensional spaces we have that there exists $\mathbb{Z}^{\text {neg }}(\varepsilon)$ a finite subset of $\mathbb{Z}$ such that for any $\varepsilon \in(-\delta, \delta)$, the following assertions hold true:

$$
\operatorname{dom}\left(L_{k}^{\text {neg }}(\varepsilon)\right)=\hat{H}_{k}^{2}\left(\mathbb{R}, \mathbb{C}^{n}\right) \cap \mathfrak{J F}_{k}\left(\operatorname{Range}\left(\Pi^{\text {neg }}(\varepsilon)\right)\right) \text { for all } k \in \mathbb{Z} \backslash \mathbb{Z}^{\text {neg }}(\varepsilon) ;
$$

$\operatorname{dom}\left(L_{k}^{\text {neg }}(\varepsilon)\right)$ is a finite codimension subspace $\hat{H}_{k}^{2}\left(\mathbb{R}, \mathbb{C}^{n}\right) \cap \mathfrak{J}_{k}\left(\operatorname{Range}\left(\Pi^{\text {neg }}(\varepsilon)\right)\right)$ for all $k \in \mathbb{Z}^{\text {neg }}(\varepsilon)$. 
From the definition of spectral projections and Hypothesis $\left(D_{\varepsilon}\right)$ we have that $\sigma\left(L^{\text {neg }}(\varepsilon)\right) \subset\{\lambda \in$ $\mathbb{C}: \operatorname{Re} \lambda<0\} \cup\{0\}$, which implies that

$$
\{\lambda \in \mathbb{C}: \operatorname{Re} \lambda>0\} \subset \rho\left(L_{k}^{\text {neg }}(\varepsilon)\right) \text { for all } k \in \mathbb{Z}, \varepsilon \in(-\delta, \delta) .
$$

In the next step we are going to prove that the semigroups generated by $L_{k}^{\text {neg }}(\varepsilon), k \in \mathbb{Z} \backslash\{0\}$, are uniformly exponentially stable. In order to formulate the result, we introduce the spaces

$$
\mathbb{T}_{k}^{p}(\varepsilon)=\hat{H}_{k}^{p}\left(\mathbb{R}, \mathbb{C}^{n}\right) \cap \mathfrak{J F}_{k}\left(\text { Range }\left(\Pi^{\text {neg }}(\varepsilon)\right)\right), \quad p=1,2 .
$$

Lemma 3.6. Assume Hypotheses (A1)-(A3), (B1), (H0)-(H6) and $\left(D_{\varepsilon}\right)$. Then, the following assertions hold true:

(i) There exists $C>0$ sufficiently large such that for any $k \in \mathbb{Z} \backslash\{0\}, \varepsilon \in(-\delta, \delta)$ the following estimate holds

$$
\left\|\left(\lambda-L_{k}^{\mathrm{neg}}(\varepsilon)\right)^{-1}\right\|_{\mathfrak{T}_{k}^{1}(\varepsilon) \rightarrow \mathfrak{C}_{k}^{1}(\varepsilon)} \leq C \text { for any } \lambda \in \mathbb{C} \text { with } \operatorname{Re} \lambda>0
$$

(ii) There exists a constant $\nu>0$, small enough such that for any $k \in \mathbb{Z} \backslash\{0\}, \varepsilon \in(-\delta, \delta)$ the following estimate holds

$$
\left\|e^{t L_{k}^{\mathrm{neg}}(\varepsilon)}\right\|_{\mathfrak{T}_{k}^{1}(\varepsilon) \rightarrow \mathfrak{C}_{k}^{1}(\varepsilon)} \leq C e^{-\zeta t} \text { for any } t \geq 0 .
$$

Proof. First, we note that we can apply the results from [Z2, Prop. 4.7] to conclude that there are two positive constants $R$ and $C$ sufficiently large and $\theta_{00}>0$ sufficiently small such that for any $\varepsilon \in(-\delta, \delta)$

$$
\left\|\left(\lambda-L_{k}(\varepsilon)\right)^{-1}\right\|_{\mathfrak{C}_{k}^{1}(\varepsilon) \rightarrow \mathfrak{C}_{k}^{1}(\varepsilon)} \leq C \text { whenever }|(k, \lambda)| \geq R, \operatorname{Re} \lambda>-\theta_{00}
$$

It follows that

$$
\left\|\left(\lambda-L_{k}^{\mathrm{neg}}(\varepsilon)\right)^{-1}\right\|_{\mathfrak{T}_{k}^{1}(\varepsilon) \rightarrow \mathfrak{C}_{k}^{1}(\varepsilon)} \leq C \text { whenever }|\lambda| \geq R, \operatorname{Re} \lambda \geq 0
$$

for any $\varepsilon \in(-\delta, \delta)$ and any $k \in \mathbb{Z} \backslash\{0\}$. From Hypothesis $\left(D_{\varepsilon}\right)$ we conclude that $\sigma\left(L^{\text {neg }}(\varepsilon)\right)=$ $\sigma_{\text {ess }}(L(\varepsilon))$, which implies that the operator $L^{\text {neg }}(\varepsilon)$ has only essential spectrum, that is $\sigma\left(L_{k}^{\text {neg }}(\varepsilon)\right)=$ $\sigma_{\text {ess }}\left(L_{k}^{\text {neg }}(\varepsilon)\right)$ for all $k \in \mathbb{Z} \backslash\{0\}$. Moreover, from (A1)-(A3) and (H3) we infer that

$$
\sup \operatorname{Re} \sigma_{\mathrm{ess}}\left(L_{k}^{\mathrm{neg}}(\varepsilon)\right) \leq \sup _{\xi \in \mathbb{R}}\left[-\frac{\theta_{0}\left(\xi^{2}+k^{2}\right)}{1+\xi^{2}+k^{2}}\right] \leq-\frac{\theta_{0}}{2}<0
$$

for any $\varepsilon \in(-\delta, \delta)$ and any $k \in \mathbb{Z} \backslash\{0\}$. We conclude that $\{\lambda \in \mathbb{C}: \operatorname{Re} \lambda \geq 0,|\lambda| \leq R\}$ is contained in $\rho\left(L_{k}^{\text {neg }}(\varepsilon)\right)$ for any $\varepsilon \in(-\delta, \delta)$ and any $k \in \mathbb{Z} \backslash\{0\}$, which implies that

$$
\left\|\left(\lambda-L_{k}^{\mathrm{neg}}(\varepsilon)\right)^{-1}\right\|_{\mathfrak{T}_{k}^{1}(\varepsilon) \rightarrow \mathfrak{C}_{k}^{1}(\varepsilon)} \leq C \text { whenever }|\lambda| \leq R, \operatorname{Re} \lambda \geq 0
$$

for any $\varepsilon \in(-\delta, \delta)$ and any $k \in \mathbb{Z} \backslash\{0\}$. Assertion (i) follows shortly from (3.38) and (3.39). Assertion (ii) follows from the Gearhart-Prüss Spectral Mapping theorem for $C_{0}$-semigroups on Hilbert spaces and the estimate (3.35).

The (right) invertibility problem for $e^{T L_{0}^{\mathrm{neg}}(\varepsilon)} \Pi(\varepsilon)-I$ was settled in [TZ3, Prop.4] (see also [TZ2, Lemma 5.10]). To formulate this result we need to introduce the function spaces $X_{1}, B_{1}, X_{2}$ and $B_{2}$ as follows:

$$
X_{1}=H_{\eta}^{2}\left(\mathbb{R}, \mathbb{C}^{n}\right)=H^{2}\left(\mathbb{R}, \mathbb{C}^{n} ; e^{\eta\left(1+\left|x_{1}\right|^{2}\right)^{1 / 2}} d x_{1}\right), \quad B_{1}=H^{1}\left(\mathbb{R}, \mathbb{C}^{n}\right),
$$

with their natural Hilbert space scalar product. Furthermore, we define

$$
X_{2}=\partial_{x_{1}} H_{2 \eta}^{1}\left(\mathbb{R}, \mathbb{C}^{n}\right) \cap X_{1}, \quad B_{2}=\partial_{x_{1}} L^{1}\left(\mathbb{R}, \mathbb{C}^{n}\right) \cap B_{1}
$$


and note that $X_{2}$ is a Hilbert space while $B_{2}$ is a Banach space. The scalar product on $X_{2}$ and the norm on $B_{2}$ are defined by

$$
\left\langle\partial_{x_{1}} f, \partial_{x_{1}} g\right\rangle_{X_{2}}=\left\langle\partial_{x_{1}} f, \partial_{x_{1}} g\right\rangle_{H_{\eta}^{2}}+\langle f, g\rangle_{H_{2 \eta}^{1}}, \quad\left\|\partial_{x_{1}} f\right\|_{B_{2}}=\|f\|_{L^{1}}+\left\|\partial_{x_{1}} f\right\|_{H^{1} 1} .
$$

Remark 3.7. Under Hypotheses (A1)-(A3), (B1), (H0)-(H6) and $\left(D_{\varepsilon}\right)$, we can choose a $\delta>0$ small enough such that the operator $e^{T L_{0}^{\text {neg }}(\varepsilon)} \Pi(\varepsilon)-I$ has a right inverse, bounded from $X_{2}$ to $X_{1}$ and from $B_{2}$ to $B_{1}$, uniformly in $(\varepsilon, T)$ for $T \in\left[T_{0}, T_{1}\right], 0<T_{0}<T_{1}<\infty$, and $\varepsilon \in(-\delta, \delta)$. Moreover, the function

$$
(\varepsilon, T) \rightarrow\left(e^{T L_{0}^{\mathrm{neg}}(\varepsilon)} \Pi(\varepsilon)-I\right)_{\mid X_{2}}^{\dagger}:(-\delta, \delta) \times\left[T_{0}, T_{1}\right] \rightarrow L\left(X_{2}, X_{1}\right) \cap L\left(B_{2}, B_{1}\right)
$$

is $\mathcal{C}^{1}$ in the $L\left(B_{2}, B_{1}\right)$ norm $^{9}$.

In the following lemma we collect the results from the previous lemmas on the invertibility of $e^{T L^{\text {neg }}(\varepsilon)} \Pi(\varepsilon)-I$. To formulate the result we define the spaces

$$
\begin{aligned}
& \mathcal{X}_{1}=\bigoplus_{k \in \mathbb{Z} \backslash\{0\}} \hat{H}_{k}^{1}\left(\mathbb{R}, \mathbb{C}^{n}\right) \oplus X_{1}, \quad \mathcal{B}_{1}=\bigoplus_{k \in \mathbb{Z} \backslash\{0\}} \hat{H}_{k}^{1}\left(\mathbb{R}, \mathbb{C}^{n}\right) \oplus B_{1}, \\
& \mathcal{X}_{2}=\bigoplus_{k \in \mathbb{Z} \backslash\{0\}} \hat{H}_{k}^{1}\left(\mathbb{R}, \mathbb{C}^{n}\right) \oplus X_{2}, \quad \mathcal{B}_{2}=\bigoplus_{k \in \mathbb{Z} \backslash\{0\}} \hat{H}_{k}^{2}\left(\mathbb{R}, \mathbb{C}^{n}\right) \oplus B_{2} .
\end{aligned}
$$

We recall the definition of $\hat{H}_{k}^{p}\left(\mathbb{R}, \mathbb{C}^{n}\right), k \in \mathbb{Z}, p=1,2$, given in (3.1) and (3.2) and the definition of $X_{1}, B_{1}, X_{2}$ and $B_{2}$ in (3.40) and(3.41). We point out that a standard inverse of the operator $e^{T L^{\Pi}(\varepsilon)} \Pi(\varepsilon)-I$ does not exist, since 0 is an eigenvalue of $L^{\Pi}(\varepsilon)$, and thus, by the spectral mapping theorem for point spectrum, of $e^{T L^{\Pi}(\varepsilon)} \Pi(\varepsilon)-I$. One can hope to obtain a right inverse, since the lack of invertibility is due to group invariance. However, due to the lack of spectral gap, this issue is nontrivial; see [TZ2, TZ3] for further discussion.

Lemma 3.8. Under Hypotheses (A1)-(A3), (B1), (H0)-(H6) and $\left(D_{\varepsilon}\right)$, we can choose $\delta>0$ small enough and $T_{0}>0$ large enough, such that the operator $e^{T L^{\Pi}(\varepsilon)} \Pi(\varepsilon)-I$ has a right inverse, bounded from $\mathcal{X}_{2}$ to $\mathcal{X}_{1}$ and from $\mathcal{B}_{2}$ to $\mathcal{B}_{1}$, uniformly in $(\varepsilon, T)$ for $T \in\left[T_{0}, T_{1}\right], T_{1}<\infty$, and $\varepsilon \in(-\delta, \delta)$. Moreover, the function

$$
(\varepsilon, T) \rightarrow\left(e^{T L^{\Pi}(\varepsilon)} \Pi(\varepsilon)-I\right)_{\mid \mathcal{X}_{2}}^{\dagger}:(-\delta, \delta) \times\left[T_{0}, T_{1}\right] \rightarrow L\left(\mathcal{X}_{2}, \mathcal{X}_{1}\right) \cap L\left(\mathcal{B}_{2}, \mathcal{B}_{1}\right)
$$

is $\mathcal{C}^{1}$ in the $L\left(\mathcal{B}_{2}, \mathcal{B}_{1}\right)$ norm.

Proof. Without loss of generality we can choose $T_{0}>0$ large enough so that $C e^{-\zeta T_{0}} \leq \frac{1}{2}$, where $C$ and $\nu$ are defined in (3.35) and (3.36). The invertibility result follows from Lemma 3.6, Remark 3.7, (3.29) and (3.30). Next, we note that using the regularity properties of $L(\varepsilon)$ we have that

$$
(\varepsilon, T) \rightarrow\left(e^{T L_{k}^{\mathrm{neg}}(\varepsilon)} \Pi(\varepsilon)\right)_{k \in \mathbb{Z} \backslash\{0\}}:(-\delta, \delta) \times\left[T_{0}, T_{1}\right] \rightarrow L\left(\bigoplus_{k \in \mathbb{Z} \backslash\{0\}} \hat{H}_{k}^{2}\left(\mathbb{R}, \mathbb{C}^{n}\right), \bigoplus_{k \in \mathbb{Z} \backslash\{0\}} \hat{H}_{k}^{1}\left(\mathbb{R}, \mathbb{C}^{n}\right)\right)
$$

is $C^{1}$. The lemma follows shortly from Remark 3.7, (3.29), (3.30) and (3.47).

Now we are ready to apply the Lyapunov-Schmidt reduction on (3.27). We follow the procedure described in detail in [TZ2] and further developed in [TZ3]. Using the result from Lemma 3.8, we note that the infinite-dimensional part of equation (3.27), $\operatorname{Disp}_{3}\left(a_{1}, a_{2}, \tilde{\mathbf{v}}_{0}, \varepsilon, T\right)=0$, is equivalent to

$$
\tilde{\mathbf{v}}_{0}=\left(I-e^{T L^{\Pi}(\varepsilon)} \Pi(\varepsilon)\right)_{\mid \mathcal{X}_{2}}^{\dagger} \mathbf{N}_{3}\left(a_{1}, a_{2}, \tilde{\mathbf{v}}_{0}, \varepsilon, T\right)+h,
$$

\footnotetext{
${ }^{9}$ Throughout this paper we use $A^{\dagger}$ to denote the right inverse of linear operator $A$
} 
for $h \in \operatorname{ker}\left(I-e^{T L^{\Pi}(\varepsilon)} \Pi(\varepsilon)\right) \cap \mathcal{X}_{1}$, where $\mathbf{N}_{j}, j=1,2,3$, denote the nonlinear integral terms from the definition of Disp in (3.24)-(3.26). Another key element of the analysis in [TZ2] is to use the (right) invertibility result from Lemma 3.8 to show that $\left(I-e^{T L^{\Pi}(\varepsilon)} \Pi(\varepsilon)\right)_{\mid \mathcal{X}_{2}}^{\dagger}$ is well-defined and bounded on Range $\mathbf{N}_{3}$ and then prove contractivity by the quadratic bounds of $\mathbf{N}_{3}$. In the next lemma we collect some estimates satisfied by the nonlinearities $\mathbf{N}_{j}, j=1,2,3$.

Lemma 3.9. Under Hypotheses (A1)-(A3), (B1), (H0)-(H6) and $\left(D_{\varepsilon}\right)$, the function $\mathbf{N}_{j}: \mathbb{C}^{2} \times$ $H^{2}\left(\mathbb{R} \times[-\pi, \pi], \mathbb{C}^{n}\right) \times(-\delta, \delta) \times(0, \infty) \rightarrow \mathbb{C}, j=1,2$, is quadratic order and $C^{1}$. Moreover, for any $M>0$ the map $\mathbf{N}_{3}: \mathbb{C}^{2} \times B_{\mathcal{X}_{1}}(0, M) \times(-\delta, \delta) \times(0, \infty) \rightarrow \mathcal{X}_{2}$ is quadratic order and $C^{1}$ from $\mathbb{C}^{2} \times \mathcal{B}_{1} \times(-\delta, \delta) \times(0, \infty)$ to $\mathcal{B}_{2}$. More precisely the following estimates hold true:

$$
\begin{aligned}
& \left|\mathbf{N}_{j}\left(a_{1}, a_{2}, \tilde{\mathbf{v}}_{0}, \varepsilon, T\right)\right|+\left|\partial_{\varepsilon, T} \mathbf{N}_{j}\left(a_{1}, a_{2}, \tilde{\mathbf{v}}_{0}, \varepsilon, T\right)\right| \leq c\left(\left|a_{1}\right|+\left|a_{2}\right|+\left\|\tilde{\mathbf{v}}_{0}\right\| \|_{\mathcal{B}_{1}}\right)^{2} \\
& \left|\partial_{a_{k}} \mathbf{N}_{j}\left(a_{1}, a_{2}, \tilde{\mathbf{v}}_{0}, \varepsilon, T\right)\right|+\left\|\partial_{\tilde{\mathbf{v}}_{0}} \mathbf{N}_{j}\left(a_{1}, a_{2}, \tilde{\mathbf{v}}_{0}, \varepsilon, T\right)\right\|_{\mathcal{B}_{1}} \leq c\left(\left|a_{1}\right|+\left|a_{2}\right|+\left\|\tilde{\mathbf{v}}_{0}\right\|_{\mathcal{B}_{1}}\right) \\
& \left|\mathbf{N}_{3}\left(a_{1}, a_{2}, \tilde{\mathbf{v}}_{0}, \varepsilon, T\right)\right|+\left\|\partial_{\varepsilon, T} \mathbf{N}_{3}\left(a_{1}, a_{2}, \tilde{\mathbf{v}}_{0}, \varepsilon, T\right)\right\|_{\mathcal{B}_{2}} \leq c\left(\left|a_{1}\right|+\left|a_{2}\right|+\left\|\tilde{\mathbf{v}}_{0}\right\|_{\mathcal{X}_{1}}\right)^{2} \\
& \left\|\partial_{a_{k}} \mathbf{N}_{3}\left(a_{1}, a_{2}, \tilde{\mathbf{v}}_{0}, \varepsilon, T\right)\right\|_{\mathcal{B}_{2}}+\left\|\partial_{\tilde{\mathbf{v}}_{0}} \mathbf{N}_{3}\left(a_{1}, a_{2}, \tilde{\mathbf{v}}_{0}, \varepsilon, T\right)\right\|_{L\left(\mathcal{B}_{1}, \mathcal{B}_{2}\right)} \leq c\left(\left|a_{1}\right|+\left|a_{2}\right|+\left\|\tilde{\mathbf{v}}_{0}\right\|_{\mathcal{X}_{1}}\right),
\end{aligned}
$$

for any $j=1,2$ and $k=1,2$, whenever $\left\|\tilde{\mathbf{v}}_{0}\right\|_{\mathcal{X}_{1}} \leq M$.

Proof. These estimates follow shortly from the estimates from Proposition 2.2 and the variation of constants formulas of $\mathbf{N}_{j}$, given in (3.24)-(3.26).

We conclude this section with this a result describing $\operatorname{ker}\left(I-e^{T L^{\Pi}(\varepsilon)}\right)$.

Lemma 3.10. Under Hypotheses (A1)-(A3), (B1), (H0)-(H6) and $\left(D_{\varepsilon}\right)$, there exist smooth functions $h_{1}, \ldots, h_{\ell}:(-\delta, \delta) \rightarrow H^{2}\left(\mathbb{R} \times[-\pi, \pi], \mathbb{C}^{n}\right)$ such that $\left\{h_{j}(\varepsilon): j=1, \ldots, \ell\right\}$ is a basis of $\operatorname{ker}\left(I-e^{T L^{\Pi}(\varepsilon)} \Pi(\varepsilon)\right)$ of dimension $\ell$ (defined in (H4)) for all $T>0$.

Proof. From hypothesis $\left(D_{\varepsilon}\right)$ (ii) one can readily infer the existence of an $\ell$-dimensional, $\varepsilon$-smooth basis of ker $L^{\Pi}(\varepsilon)$. The lemma follows shortly by using the intricate connections between $\operatorname{ker} L^{\Pi}(\varepsilon)$ and $\operatorname{ker}\left(I-e^{T L^{\Pi}(\varepsilon)} \Pi(\varepsilon)\right)$ that one can readily check by using elementary semigroup theory.

\section{Proof of the MAIN RESUlt}

In this section we collect the result from the previous sections to prove the existence of an $O(2)$ Hopf bifurcation from our one-parameter family of standing viscous planar shocks. First, we solve for $\tilde{\mathbf{v}}_{0}$ in the infinite-dimensional equation $\operatorname{Disp}_{3}=0$.

Lemma 4.1. Under Hypotheses (A1)-(A3), (B1), (HO)-(H6) and $\left(D_{\varepsilon}\right)$, there exists $\delta>0$ small enough and a map $\mathbf{z}: \mathbb{C}^{2} \times(-\delta, \delta) \times\left[T_{0}, T_{1}\right] \times \mathbb{R}^{\ell} \rightarrow \mathcal{X}_{1}$ that is $C^{1}$ from $\mathbb{C}^{2} \times(-\delta, \delta) \times\left[T_{0}, T_{1}\right] \times \mathbb{R}^{\ell}$ to $\mathcal{B}_{1}$ such that for any $a_{1}, a_{2} \in B_{\mathbb{C}}(0, \delta), \varepsilon \in(-\delta, \delta)$ and $T \in\left[T_{0}, T_{1}\right]$, the local solutions of equation $\operatorname{Disp}_{3}\left(a_{1}, a_{2}, \tilde{\mathbf{v}}_{0}, \varepsilon, T\right)=0$ are given by $\tilde{\mathbf{v}}_{0}=\mathbf{z}\left(a_{1}, a_{2}, \varepsilon, T, b_{1}, \ldots, b_{\ell}\right)$ for some $\left(b_{1}, \ldots, b_{\ell}\right) \in \mathbb{R}^{\ell}$.

Proof. We define the map $\mathbf{F}: \mathbb{C}^{2} \times \mathcal{X}_{1} \times(-\delta, \delta) \times\left[T_{0}, T_{1}\right] \times \mathbb{R}^{\ell} \rightarrow \mathcal{X}_{1}$ by

$$
\mathbf{F}\left(a_{1}, a_{2}, \tilde{\mathbf{v}}_{0}, \varepsilon, T, b_{1}, \ldots, b_{\ell}\right)=\left(I-e^{T L^{\Pi}(\varepsilon)}\right)_{\mid \mathcal{X}_{2}}^{\dagger} \mathbf{N}_{3}\left(a_{1}, a_{2}, \tilde{\mathbf{v}}_{0}, \varepsilon, T\right)+\sum_{j=1}^{\ell} b_{j} h_{j}(\varepsilon) .
$$

From (3.48) we have that the equation $\operatorname{Disp}_{3}\left(a_{1}, a_{2}, \tilde{\mathbf{v}}_{0}, \varepsilon, T\right)=0$ is equivalent to the fixed point equation

$$
\tilde{\mathbf{v}}_{0}=\mathbf{F}\left(a_{1}, a_{2}, \tilde{\mathbf{v}}_{0}, \varepsilon, T, b_{1}, \ldots, b_{\ell}\right),
$$

for some $b=\left(b_{1}, \ldots, b_{\ell}\right) \in \mathbb{R}^{\ell}$. Using the results from [TZ2, Section 2] and [TZ3, Section 4] we can show that the map $\mathbf{F}$ is bounded from $\mathbb{C}^{2} \times \mathcal{X}_{1} \times(-\delta, \delta) \times\left[T_{0}, T_{1}\right] \times \mathbb{R}^{\ell}$ to $\mathcal{X}_{1}$ and $C^{1}$ from 
$\mathbb{C}^{2} \times \mathcal{B}_{1} \times(-\delta, \delta) \times\left[T_{0}, T_{1}\right] \times \mathbb{R}^{\ell}$ to $\mathcal{X}_{1}$. Moreover, using the results from Lemma 3.8 and Lemma 3.9 we readily obtain appropriate estimates on $\mathbf{F}$ and its partial derivatives. Using again the results from [TZ2, Section 2] we infer by a Contraction Mapping/Implicit Function Theorem type argument that there exists a map z : $\mathbb{C}^{2} \times(-\delta, \delta) \times\left[T_{0}, T_{1}\right] \times \mathbb{R}^{\ell} \rightarrow \mathcal{X}_{1}$ that is $C^{1}$ from $\mathbb{C}^{2} \times(-\delta, \delta) \times\left[T_{0}, T_{1}\right] \times \mathbb{R}^{\ell}$ to $\mathcal{B}_{1}$ such that $\tilde{\mathbf{v}}_{0}=\mathbf{z}\left(a_{1}, a_{2}, \varepsilon, T, b_{1}, \ldots, b_{\ell}\right)$ uniquely solves (4.2) locally. This proves the lemma.

Remark 4.2. Since the function $\mathbf{z}$ is $C^{1}$ from $\mathbb{C}^{2} \times(-\delta, \delta) \times\left[T_{0}, T_{1}\right] \times \mathbb{R}^{\ell}$ to $\mathcal{B}_{1}$, we can use the results from Lemma 3.8 and Lemma 3.9 to infer that

$$
\mathbf{z}(0,0,0, T, 0, \ldots, 0)=0 .
$$

Moreover, by differentiating with respect to $T$ in (4.2), one can easily check that

$$
\partial_{T} \mathbf{z}(0,0,0, T, 0, \ldots, 0)=0 .
$$

At this point we note that to solve the equation Disp $=0$ it is enough to solve a system of two scalar (complex) equations, with variables $a_{1}, a_{2}, \varepsilon, T$ and parameters $b_{1}, \ldots, b_{\ell}$. Next, we choose $k_{*} \in \mathbb{Z}$, with $\left|k_{*}\right|$ large enough such that $\frac{2 k_{*} \pi}{\omega(0)} \in\left(T_{0}, T_{1}\right)$. Let $T_{*}:(-\delta, \delta) \rightarrow \mathbb{R}$ be the function defined by $T_{*}(\varepsilon)=\frac{2 k_{*} \pi}{\omega(\varepsilon)}$. We plug in

$$
\tilde{\mathbf{v}}_{0}=\mathbf{z}\left(a_{1}, a_{2}, \varepsilon, T, b_{1}, \ldots, b_{\ell}\right) \quad \text { and } \quad T=T_{*}(\varepsilon)(1+\mu), \quad \mu \in(-\delta, \delta)
$$

in (3.24) and (3.25) to obtain the system

$$
\left\{\begin{array}{l}
\tilde{\mathbf{N}}_{1}\left(a_{1}, a_{2}, \varepsilon, \mu, b_{1}, \ldots, b_{\ell}\right)=0 \\
\tilde{\mathbf{N}}_{2}\left(a_{1}, a_{2}, \varepsilon, \mu, b_{1}, \ldots, b_{\ell}\right)=0
\end{array} .\right.
$$

To solve the remaining $\mathbb{C}^{2}$ system in variables $a_{1}, a_{2}$, with bifurcation parameters $\varepsilon$ and $\mu$, and involving parameters $\left(b_{1}, \ldots, b_{\ell}\right) \in(-\delta, \delta)^{\ell}$, we need to identify the symmetries that are satisfied by this system, which are inherited from the original system (2.2) or its reformulation (3.22).

Lemma 4.3. Under Hypotheses (A1)-(A3), (B1), (HO)-(H6) and $\left(D_{\varepsilon}\right)$, the finite-dimensional system (4.6) is invariant in the variables $\left(a_{1}, a_{2}\right)$ under the symmetry $\left(z_{1}, z_{2}\right) \rightarrow\left(z_{2}, z_{1}\right)$ and the rotation $\left(z_{1}, z_{2}\right) \rightarrow\left(z_{1} e^{\mathrm{i} \theta}, z_{2} e^{-\mathrm{i} \theta}\right)$ for any $\theta \in \mathbb{R}$.

Proof. We recall that the system (2.2) is invariant under the symmetry $S$ and the non-degenerate rotation group $\{R(\theta)\}_{\theta \in \mathbb{R}}$. Therefore, the group actions of $S$ and the $C_{0}$-group $\{R(\theta)\}_{\theta \in \mathbb{R}}$ are inherited on the eigenspaces $\Sigma_{ \pm}(\varepsilon)$ associated to the crossing eigenvalues $\lambda_{ \pm}(\varepsilon)$. From Lemma 3.2 and since $S$ is a symmetry, we infer that the group action of $S$ on the eigenspaces $\Sigma_{ \pm}(\varepsilon)$ is isomorphic to the transformation $\tilde{S}: \mathbb{C}^{2} \rightarrow \mathbb{C}^{2}$ defined by $\tilde{S}\left(z_{1}, z_{2}\right)=\left(z_{2}, z_{1}\right)$. Using Lemma 3.2 and (3.12), we conclude that for any $\theta \in \mathbb{R}$ the group action of $R(\theta)$ on the eigenspaces $\Sigma_{ \pm}(\varepsilon)$ is isomorphic to the transformation $\tilde{R}(\theta): \mathbb{C}^{2} \rightarrow \mathbb{C}^{2}$ defined by $\tilde{R}(\theta)\left(z_{1}, z_{2}\right)=\left(z_{1} e^{\mathrm{i} \alpha(\varepsilon) \theta}, z_{2} e^{-\mathrm{i} \alpha(\varepsilon) \theta}\right)$. It follows that the system (4.6) is invariant in the variables $\left(a_{1}, a_{2}\right) \in \mathbb{C}^{2}$ under the transformations $\tilde{S}$ and $\tilde{R}(\theta)$ for all $\theta \in \mathbb{R}$. Making the change of variables $\theta \rightarrow \frac{\theta}{\alpha(\varepsilon)}$, the lemma follows shortly.

It was shown in [TZ3, Rmk 13] that one can easily improve the $C^{1}$-regularity of the map $\mathbf{z}$ by choosing the spaces $X_{j}, \mathrm{j}=1,2$ (defined in (3.40) and (3.41)), appropriately.

Remark 4.4. By choosing the space $X_{1}=H_{\eta}^{4}(\mathbb{R}, \mathbb{C})=H^{4}\left(\mathbb{R}, \mathbb{C}^{n} ; e^{\eta\left(1+\left|x_{1}\right|^{2}\right)^{1 / 2}} d x_{1}\right)$ and $X_{2}=$ $\partial_{x_{1}} H_{2 \eta}^{1}\left(\mathbb{R}, \mathbb{C}^{n}\right) \cap X_{1}$, one can use the same analysis from [TZ2, TZ3] to prove that $\mathbf{z}$ is of class $C^{2}$. More generally, we can strengthen the result by proving that the map $\mathbf{z}$ is of class $C^{m}$ where $\nu=2 m+1$ in (H0). Since $\nu \geq 5$, we have that $\mathbf{z}$ is (at least) of class $C^{3}$ from $\mathbb{C}^{2} \times(-\delta, \delta) \times\left[T_{0}, T_{1}\right] \times \mathbb{R}^{\ell}$ to $\mathcal{B}_{1}$. Therefore, we conclude that the functions $\tilde{\mathbf{N}}_{j}, j=1,2$, are of class (at least) $C^{3}$ from $B_{\mathbb{C}}(0, \delta)^{2} \times(-\delta, \delta)^{2+\ell}$ to $\mathbb{C}$. 
In the next lemma we exploit the fact that $\tilde{\mathbf{N}}_{j}, j=1,2$, are of class $C^{3}$ by expanding these functions to cubic order.

Lemma 4.5. Under Hypotheses (A1)-(A3), (B1), (H0)-(H6) and $\left(D_{\varepsilon}\right)$, there exists $\delta>0$ small enough, two non-zero real constants $\varkappa, \chi \neq 0$ and smooth functions $\Lambda, \Gamma:(-\delta, \delta)^{\ell} \rightarrow \mathbb{C}$ such that

$$
\begin{aligned}
& \tilde{\mathbf{N}}_{1}\left(a_{1}, a_{2}, \varepsilon, \mu, b_{1}, \ldots, b_{\ell}\right)=a_{1}\left(\varkappa \varepsilon+\mathrm{i} \chi \mu+\Lambda\left(b_{1}, \ldots, b_{\ell}\right)\left|a_{1}\right|^{2}+\Gamma\left(b_{1}, \ldots, b_{\ell}\right)\left|a_{2}\right|^{2}\right)+\mathcal{O}(4), \\
& \tilde{\mathbf{N}}_{2}\left(a_{1}, a_{2}, \varepsilon, \mu, b_{1}, \ldots, b_{\ell}\right)=a_{2}\left(\varkappa \varepsilon+\mathrm{i} \chi \mu+\Gamma\left(b_{1}, \ldots, b_{\ell}\right)\left|a_{1}\right|^{2}+\Lambda\left(b_{1}, \ldots, b_{\ell}\right)\left|a_{2}\right|^{2}\right)+\mathcal{O}(4) .
\end{aligned}
$$

Proof. To find an expansion for the functions $\tilde{\mathbf{N}}_{j}, j=1,2$, we use the definition of the functions $\operatorname{Disp}_{j}, j=1,2$ given in (3.24) and (3.25) and the invariance properties of the system (4.6) proved in Lemma 4.3. From Lemma 3.9, (4.3), (4.4) and the substitution (4.5) it follows that the leading order terms in $\varepsilon$ and $\mu$ are obtained from the leading order terms of $\mathbf{D i s p}_{j}, j=1,2$, namely

$$
\left(e^{T_{*}(\varepsilon)(1+\mu) \lambda_{+}(\varepsilon)}-1\right) a_{j}=\left(e^{T_{*}(\varepsilon)(1+\mu) \gamma(\varepsilon)+2 k_{*} \pi \mathrm{i} \mu}-1\right) a_{j}, \quad j=1,2 .
$$

Taking $\varkappa=\gamma^{\prime}(0) T_{*}(0)=\frac{2 k_{*} \pi \gamma^{\prime}(0)}{\omega(0)} \neq 0$ (by Hypothesis $\left(D_{\varepsilon}\right)$ ) and $\chi=2 k_{*} \pi \neq 0$, we infer that there exist smooth functions $\Lambda_{j}, \Gamma_{j}, \Upsilon_{j}:(-\delta, \delta)^{\ell} \rightarrow \mathbb{R}$ such that

$$
\begin{aligned}
& \tilde{\mathbf{N}}_{1}\left(a_{1}, a_{2}, \varepsilon, \mu, b_{1}, \ldots, b_{\ell}\right)=a_{1}\left(\varkappa \varepsilon+\mathrm{i} \chi \mu+\Lambda_{1}\left(b_{1}, \ldots, b_{\ell}\right)\left|a_{1}\right|^{2}+\Upsilon_{1}\left(b_{1}, \ldots, b_{\ell}\right) a_{1} a_{2}\right. \\
& \left.+\Gamma_{1}\left(b_{1}, \ldots, b_{\ell}\right)\left|a_{2}\right|^{2}\right)+\overline{a_{2}}\left(+\Lambda_{2}\left(b_{1}, \ldots, b_{\ell}\right)\left|a_{1}\right|^{2}+\Upsilon_{2}\left(b_{1}, \ldots, b_{\ell}\right) \overline{a_{1} a_{2}}+\Gamma_{2}\left(b_{1}, \ldots, b_{\ell}\right)\left|a_{2}\right|^{2}\right)+\mathcal{O}(4), \\
& \tilde{\mathbf{N}}_{2}\left(a_{1}, a_{2}, \varepsilon, \mu, b_{1}, \ldots, b_{\ell}\right)=a_{2}\left(\varkappa \varepsilon+\mathrm{i} \chi \mu+\Gamma_{1}\left(b_{1}, \ldots, b_{\ell}\right)\left|a_{1}\right|^{2}+\Upsilon_{1}\left(b_{1}, \ldots, b_{\ell}\right) a_{1} a_{2}\right. \\
& \left.+\Lambda_{1}\left(b_{1}, \ldots, b_{\ell}\right)\left|a_{2}\right|^{2}\right)+\overline{a_{1}}\left(\Gamma_{2}\left(b_{1}, \ldots, b_{\ell}\right)\left|a_{1}\right|^{2}+\Upsilon_{2}\left(b_{1}, \ldots, b_{\ell}\right) \overline{a_{1} a_{2}}+\Lambda_{2}\left(b_{1}, \ldots, b_{\ell}\right)\left|a_{2}\right|^{2}\right)+\mathcal{O}(4) .
\end{aligned}
$$

Noting that there always exist reflectionally symmetric solutions $a_{1} \equiv a_{2}$, for which the situation reduces to that of a standard Hopf bifurcation, recalling that the system originates from a rotating evolutionary system in which $a_{1}, a_{2}$ rotate in a common direction with common (to linear order) speed $\omega(\varepsilon)$, and noting that periodic solutions are preserved under influence of the flow, we infer that $\Upsilon_{1}=\Lambda_{2}=\Upsilon_{2}=\Gamma_{2}=0$ must hold. For, otherwise it is easy to see that solutions $a_{1} \equiv a_{2}$ are not preserved under an approximate common rotation. Dropping the index of the functions $\Lambda_{1}$ and $\Gamma_{1}$, the lemma follows immediately.

To finish the proof of our main result we introduce the following genericity assumption:

Hypothesis 1. If $\Lambda, \Gamma:(-\delta, \delta)^{\ell} \rightarrow \mathbb{C}$ are the functions from expansion (4.7), we assume that there exists $\delta>0$ small enough such that

$$
\Lambda \neq \Gamma, \quad \operatorname{Re}(\Lambda+\Gamma) \neq 0, \quad \operatorname{Re} \Lambda \neq 0 .
$$

Proof of Theorem 1.2. From Lemma 4.1 it follows that to prove the theorem it is enough to solve the finite-dimensional system (4.6). Let $\mathbf{b}=\left(b_{1}, \ldots, b_{\ell}\right) \in(-\delta, \delta)^{\ell}$ with $|\mathbf{b}| \leq C\left|\left(a_{1}, a_{2}\right)\right|$ for some constant $C>0$. Making the substitution

$$
a_{1}=a, \quad a_{2}=\rho a, \quad a \in \mathbb{C} \text { and } \rho \in \mathbb{C} \cup\{\infty\} .
$$

in (4.6) and using the results from Lemma 4.5, we obtain the equivalent system:

$$
\left\{\begin{array}{c}
a\left(\varkappa \varepsilon+\mathrm{i} \chi \mu+\Lambda(\mathbf{b})|a|^{2}+\Gamma(\mathbf{b})|a|^{2}|\rho|^{2}\right)+\mathcal{O}(4)=0 \\
a \rho\left(\varkappa \varepsilon+\mathrm{i} \chi \mu+\Gamma(\mathbf{b})|a|^{2}+\Lambda(\mathbf{b})|a|^{2}|\rho|^{2}\right)+\mathcal{O}(4)=0
\end{array} .\right.
$$

There are three cases of interest: when $a \neq 0$ and $\rho$ is bounded and bounded away from 0 and when $|\rho|<<1$ or $|\rho|>>1$. We are going to treat all of these cases separately. 
Case 1. $a \neq 0$ and there exists $C>0$ such that $\frac{1}{C} \leq|\rho| \leq C$.

Multiplying the first equation of the system (4.11) by $\rho$ and subtracting it from the second equation, we obtain the equation:

$$
a \rho\left((\Lambda(\mathbf{b})-\Gamma(\mathbf{b}))|a|^{2}\left(1-|\rho|^{2}\right)+\mathcal{O}(4)=0 .\right.
$$

Since in this case $\rho$ is bounded and bounded away from 0 we can divide this equation by $a \rho|a|^{2}$ to obtain the equation:

$$
\left((\Lambda(\mathbf{b})-\Gamma(\mathbf{b}))\left(1-|\rho|^{2}\right)+\mathcal{O}(a)=0 .\right.
$$

Since by Hypothesis 1 we have that $\Lambda(\mathbf{b}) \neq \Gamma(\mathbf{b})$ from (4.12) we infer that $|\rho|=1+\mathcal{O}(a)$. Substituting back in the first equation of (4.11) and using that $a \neq 0$ we obtain that

$$
\varkappa \varepsilon+\mathrm{i} \chi \mu+(\Lambda(\mathbf{b})+\Gamma(\mathbf{b}))|a|^{2}+\mathcal{O}\left(a^{3}\right)=0 .
$$

Depending on model parameters (specifically, the relative signs of $\varkappa$ and $\operatorname{Re}(\Lambda+\Gamma)$ ), this will occur for $\varepsilon$ positive (supercritical case) or negative (subcritical case). Taking the real part in the above equation and since $\varkappa \neq 0$ by Lemma 4.5 and $\operatorname{Re}(\Lambda(\mathbf{b})+\Gamma(\mathbf{b})) \neq 0$ by Hypothesis 1 , we infer that $|a|=\mathcal{O}\left(|\varepsilon|^{1 / 2}\right)$. From Lemma 4.5 we have that $\chi \neq 0$, therefore by taking imaginary part in (4.13) we conclude that $\mu=\mathcal{O}(\varepsilon)$.

Case 2. $a \neq 0,|\rho|<<1$.

From the first equation of (4.11), by using the fact that $\rho$ is small, we obtain the equation

$$
\varkappa \varepsilon+\mathrm{i} \chi \mu+\left(\Lambda(\mathbf{b})+|\rho|^{2} \Gamma(\mathbf{b})\right)|a|^{2}+\mathcal{O}\left(a^{3}\right)=0 .
$$

Since $\operatorname{Re} \Lambda(\mathbf{b}) \neq 0$, by Hypothesis 1 , we have that $\operatorname{Re}\left(\Lambda(\mathbf{b})+|\rho|^{2} \Gamma(\mathbf{b})\right) \neq 0$, by continuity. Taking real and imaginary part in (4.14) and using that $\varkappa, \chi \neq 0$, we infer that $|a|=\mathcal{O}\left(|\varepsilon|^{1 / 2}\right)$ and $\mu=\mathcal{O}(\varepsilon)$.

Case 3. $a \neq 0,|\rho|>>1$.

From the second equation of (4.11) and since $1 / \rho$ is small, we obtain the equation

$$
\varkappa \varepsilon+\mathrm{i} \chi \mu+\left(\Lambda(\mathbf{b})+\frac{1}{|\rho|^{2}} \Gamma(\mathbf{b})\right)|a|^{2}+\mathcal{O}\left(a^{3}\right)=0 .
$$

Using Hypothesis 1 again, we have that $\operatorname{Re}\left(\Lambda(\mathbf{b})+\frac{1}{|\rho|^{2}} \Gamma(\mathbf{b})\right) \neq 0$, by continuity. Arguing just as in the previous cases, by taking real and imaginary part in (4.15), we conclude again that $|a|=\mathcal{O}\left(|\varepsilon|^{1 / 2}\right)$ and $\mu=\mathcal{O}(\varepsilon)$. Summarizing, we conclude that any solution of (4.6) satisfies one of the conditions

$$
\text { (i) }\left|a_{1}\right|=\mathcal{O}\left(|\varepsilon|^{1 / 2}\right) \text { and } a_{2}=e^{\mathrm{i} \theta} a_{1}+\mathcal{O}\left(a_{1}^{2}\right),
$$

Moreover, whenever we have a solution of (4.6) we have that $\mu=\mathcal{O}(\varepsilon)$. Making the change of variables

$$
\tilde{a}_{1}=\frac{a_{1}}{|\varepsilon|^{1 / 2}}, \quad \tilde{a}_{2}=\frac{a_{2}}{|\varepsilon|^{1 / 2}}, \quad \tilde{\mu}=\frac{\mu}{\varepsilon}
$$

in (4.6) we obtain the system:

$$
\left\{\begin{array}{l}
\tilde{a}_{1}\left(\varkappa \operatorname{sgn}(\varepsilon)+\mathrm{i} \chi \tilde{\mu}+\Lambda(\mathbf{b})\left|\tilde{a}_{1}\right|^{2}+\Gamma(\mathbf{b})\left|\tilde{a}_{2}\right|^{2}\right)+\mathcal{O}\left(|\varepsilon|^{1 / 2}\right)=0 \\
\tilde{a}_{2}\left(\varkappa \operatorname{sgn}(\varepsilon)+\mathrm{i} \chi \tilde{\mu}+\Gamma(\mathbf{b})\left|\tilde{a}_{1}\right|^{2}+\Lambda(\mathbf{b})\left|\tilde{a}_{2}\right|^{2}\right)+\mathcal{O}\left(|\varepsilon|^{1 / 2}\right)=0
\end{array} .\right.
$$

Since the functions $\tilde{\mathbf{N}}_{j}, j=1,2$ are of class $C^{3}$, by Remark 4.4, it follows that $\mathcal{O}\left(|\varepsilon|^{1 / 2}\right)$ terms in (4.18) are of $C^{3}$ class in $\tilde{a}_{1}, \tilde{a}_{2}$ and $\tilde{\mu}$. Since system (4.18) is equivalent to the system (4.6) which 
is rotationally invariant by Lemma 4.3 , it inherits the property of rotational invariance, and in addition the property that $\left(\tilde{a}_{1}, \tilde{a}_{2}\right)=(0,0)$ is always a solution.

Setting $\varepsilon=0$ in (4.18), we reduce to (a rescaled version of) the truncated cubic system discussed in $§ 1.2 .1$, which has under nondegeneracy assumptions (4.9) precisely four families of solutions:

$$
\left(\tilde{a}_{1}, \tilde{a}_{2}\right)=(0,0),\left(a_{*}, 0\right),\left(0, a_{*}\right),\left(a_{\natural}, e^{\mathrm{i} \theta} a_{\natural}\right),
$$

$\theta \in \mathbb{R}$, of which the last three are nontrivial equilibria bifurcating from the first, zero equilibrium. Depending on model parameters, these will occur variously for $\varepsilon>0$ (supercritical case) or $\varepsilon<0$ (subcritical case), according to the rule $\operatorname{sgn}(\varepsilon)=-\left(\operatorname{Re} \Lambda(\mathbf{b})\left|\tilde{a}_{1}\right|^{2}+\operatorname{Re} \Gamma(\mathbf{b})\left|\tilde{a}_{2}\right|^{2}\right) / \varkappa$, where the righthand side is, variously, $-(\operatorname{Re} \Lambda(\mathbf{b})+\operatorname{Re} \Gamma(\mathbf{b})) a_{*}^{2} / \varkappa,-(\operatorname{Re} \Lambda(\mathbf{b})) / \varkappa$, or $-(\operatorname{Re} \Gamma(\mathbf{b}) / \varkappa$. Fixing $\mathbf{b}=0$, we find by a combination of Implicit Function arguments and symmetry considerations that each of these families continues uniquely under small perturbations in $|\varepsilon|^{1 / 2}$, to give the claimed exact solutions of the full system obtained by Lyapunov-Schmidt reduction using the choice of right inverse corresponding to $\mathbf{b}=0$. These computations are carried out in Appendix B.

It remains to deal with the $\ell$-fold indeterminacy associated with parameter $\mathbf{b}$, induced by the $\ell$-fold kernel of the one-dimensional linearized operator $L_{0}(\varepsilon)$. This may be accounted for as follows, using the method introduced for that purpose in [TZ2]. First, we make the change of variables $\tilde{b}_{j}=\frac{b_{j}}{\left|\left(a_{1}, a_{2}\right)\right|}, j=1,2$, ensuring for fixed $\tilde{\mathbf{b}}$ that $|\mathbf{b}| \leq C\left|\left(a_{1}, a_{2}\right)\right|$ as required for our arguments above; by the same arguments, we obtain thereby a unique family of solutions perturbing from the $\varepsilon=0$ solutions for each choice of $\tilde{\mathbf{b}}$, thus obtaining an $\ell$-dimensional cone of distinct solutions above each solution for $\mathbf{b}=0$, which are the unique solutions lying within the cone $\left\{\left(a_{1}, a_{2}, \tilde{\mathbf{v}}_{0}\right):\left\|\tilde{\mathbf{v}}_{0}\right\| \mathcal{X}_{1} \leq C\left|\left(a_{1}, a_{2}\right)\right|\right\}$, for some $C>0$; moreover, these cones may be extended to smooth $\ell$-dimensional manifolds of solutions by perturbing about different choices of background wave in the $\ell$-dimensional manifold of stationary shock solutions with the same endpoints, thus obtaining a family of nearby problems to which the same arguments uniformly apply. Finally, applying [TZ2, Prop. 2.20] we find that any periodic solution of (3.22) can be shifted by such a change of coordinates so as to originate in the cone $\left\{\left(a_{1}, a_{2}, \tilde{\mathbf{v}}_{0}\right):\left\|\tilde{\mathbf{v}}_{0}\right\|_{\mathcal{X}_{1}} \leq C\left|\left(a_{1}, a_{2}\right)\right|\right\}$, for some such nearby problem. Thus, we can infer uniqueness of the full $\ell$-parameter families of solutions as in [TZ2, Cor. 2.21].

Characterization as traveling waves. Finally, we verify in the Lax case, $\ell=1$, that "traveling-wave" type solutions close to $\left(\tilde{a}_{1}, \tilde{a}_{2}\right)=\left(a_{*}, 0\right)$ or $\left(0, a_{*}\right)$ are indeed traveling waves with respect to $x_{2}$. This may be seen by the computation carried out in Appendix B showing that these types of solutions are unique up to rotational invariance, i.e., up to translation with respect to $x_{2}$, for each fixed $\mathbf{b}$. On the other hand, time-translates of any such solution give a nearby periodic solution (specifically, nearby in rescaled coordinates), which must therefore be a "traveling-type" solution for some nearby choice of $\mathbf{b}$. Recalling, for the Lax case $\ell=1$, that change in $\mathbf{b}$ corresponds simply to translation in $x_{1}$, we find therefore that time-translates of "traveling-type" solutions correspond to translates in $x=\left(x_{1}, x_{2}\right)$, from which it is readily seen (by substitution in the original PDE) that they are traveling waves $h^{\varepsilon}\left(x_{1}-c t, x_{2}-d t\right)$ in $x_{1}$ and $x_{2}$. But, time-periodicity, plus the fact that the background standing shocks by our assumptions are not periodic in $x_{1}$, implies that $c$ must vanish, leaving the conclusion that the solution must be a traveling wave in $x_{2}$ alone.

Remark 4.6. The above displacement map argument substitutes in our setting for the standard approaches used to treat $O(2)$ Hopf bifurcation in other settings, namely, the center-manifold/normal forms approach, not available to us because in absence of spectral gap we have no readily available center manifold (possibly even nonexisting as far as we know); and the spatial dynamics approach, or Lyapunov-Schmidt reduction of the evolution equations recast in the class of time-periodic functions, (the original approach for ODE given in [GSS]), again not applicable to our framework based on the time-T map and the "reverse temporal dynamics" approach of [TZ3]. Both of these standard 
approaches rely on $O(2) \times S^{1}$ symmetry, with the additional $S^{1}$ symmetry imposed, respectively, by normal form reduction and time-periodicity. It is interesting that we do not need full $S^{1}$ symmetry in our argument, substituting similar but less detailed information coming from the origins of the time- $T$ map as an evolution problem to obtain the key property $\Upsilon_{1}=\Lambda_{2}=\Upsilon_{2}=\Gamma_{2}=0$. Though we do not treat it in our analysis, spectral stability information for the bifurcating waves should in principle be readily available from the reduced time- $T$ solution map (4.6) with $\varepsilon, \mu$ held fixed.

\section{GAS DYNAMiCS AND MHD}

Finally, we comment briefly on the cases of gas dynamics or MHD, to which our analysis does not apply. In Eulerian coordinates, the 2-D compressible Navier-Stokes equations are [Ba, Da, Sm]:

$$
\begin{aligned}
\rho_{t}+(\rho u)_{x}+(\rho v)_{y}= & 0 \\
(\rho u)_{t}+\left(\rho u^{2}+p\right)_{x}+(\rho u v)_{y}= & (2 \mu+\eta) u_{x x}+\mu u_{y y}+(\mu+\eta) v_{x y}, \\
(\rho v)_{t}+(\rho u v)_{x}+\left(\rho v^{2}+p\right)_{y}= & \mu v_{x x}+(2 \mu+\eta) v_{y y}+(\mu+\eta) u_{y x}, \\
(\rho E)_{t}+(\rho u E+u p)_{x}+(\rho v E+v p)_{y}= & \left(\kappa T_{x}+(2 \mu+\eta) u u_{x}+\mu v\left(v_{x}+u_{y}\right)+\eta u v_{y}\right)_{x} \\
& +\left(\kappa T_{y}+(2 \mu+\eta) v v_{y}+\mu u\left(v_{x}+u_{y}\right)+\eta v u_{x}\right)_{y}
\end{aligned}
$$

where $\rho$ is density, $u$ and $v$ are the fluid velocities in $x$ and $y$ directions, $p$ is pressure, $T$ is temperature, $E=e+\frac{u^{2}}{2}+\frac{v^{2}}{2}$ is specific energy, $e$ is specific internal energy, $\frac{u^{2}}{2}+\frac{v^{2}}{2}$ is kinetic energy, and the constants $\mu>0$ and $|\eta| \leq \mu$ and $\kappa>0$ are coefficients of first ("dynamic") and second viscosity and heat conductivity. The equations are closed by equations of state

$$
p=p(\rho, T), \quad e=e(\rho, T) .
$$

Here, $x$ and $y$, are coordinates in a rest frame, and $t \geq 0$ is time. For common fluids and gases in normal conditions, the polytropic gas laws $p=\Gamma \rho e, e=C_{v} T$ give a good fit to experimental observations, where $\Gamma>0$ and $C_{v}>0$ are constants depending on the gas [Ba]. The MHD equations feature an additional coupling to a magnetic field vector; our discussion applies also in that case.

5.1. Lagrangian formulation. Equations (5.1) may be converted to Lagrangian coordinates as follows [A, Da, DM, HT]. Let $X, Y$ denote a reference configuration, and $T=t$, and define particle paths $(x, y)(X, Y, t)$ by $\partial_{t}(x, y)=(u, v),\left.(x, y)\right|_{t=0}=\left(x_{0}, y_{0}\right)(X, Y)$ for some choice of $\left(x_{0}, y_{0}\right)(\cdot)$ Denote by $F_{j k}$ the entries of

$$
F:=\frac{\partial(x, y)}{\partial(X, Y)}:=\left(\begin{array}{ll}
\frac{\partial x}{\partial X} & \frac{\partial x}{\partial Y} \\
\frac{\partial y}{\partial X} & \frac{\partial y}{\partial Y}
\end{array}\right) .
$$

A great simplification is obtained if one can choose $\left(x_{0}, y_{0}\right)$ so that $\left.\left.\operatorname{det} F\right|_{t=0} \equiv(1 / \rho)\right|_{t=0}$, as can always be done for $L_{\text {loc }}^{1}$ data in 1-D or an $L^{1}$ multi-D perturbation thereof- in particular for the perturbed planar shocks considered here. In this case, the values $(x, y)(X, Y, t)$ may be considered as deformations of an initially uniform-density rest configuration, and equations (5.1) reduce to a special case of thermoviscoelasticity, with $e$ depending only on $\operatorname{det} F=1 / \rho$ and entropy $S$ :

$$
\begin{aligned}
\left(F_{11}\right)_{t}-u_{X}=0, \quad\left(F_{12}\right)_{t}-u_{Y}=0, \quad\left(F_{21}\right)_{t}-v_{X} & =0, \quad\left(F_{22}\right)_{t}-v_{Y}=0, \\
u_{t}+\left(F_{22} p\right)_{X}-\left(F_{21} p\right)_{Y} & =2 \text { nd-order derivative terms, } \\
v_{t}-\left(F_{12} p\right)_{X}+\left(F_{11} p\right)_{Y} & =2 \text { nd-order derivative terms, } \\
E_{t}-\left(F_{12} v p\right)_{X}+\left(F_{11} v p\right)_{Y}+\left(F_{22} u p\right)_{X}-\left(F_{21} u p\right)_{Y} & =2 \text { nd-order derivative terms, }
\end{aligned}
$$

together with the compatibility conditions (preserved by time evolution):

$$
\partial_{Y} F_{11}-\partial_{X} F_{12}=0 ; \quad \partial_{Y} F_{21}-\partial_{X} F_{22}=0 .
$$


Here, we have omitted the description of the complicated (but divergence-form) righthand sides involving second-order derivative terms involving transport effects, not needed for our discussion.

5.2. Coordinate-ambiguity. The augmented system approach followed in this paper works for the difficult case of thermoviscoelasticity, but it does not work for the apparently simpler case of gas dynamics. The reason: for gas dynamics, the "contingent" entropy of the enlarged system is only nonstrictly convex and the equations fail to be symmetric, etc. At an operational level, this is because there is no penalty on shear strains, giving neutral directions in the associated entropy function. In fact, the problem is deeper than that- it reflects the fact that Lagrangian gas dynamic equations have an infinite-dimensional family of invariances consisting of all volume preserving maps of the spatial coordinate. (Since pressure depends only on density= reciprocal of the Jacobian of the deformation map, there can be no change under Jacobian-preserving transformations.)

This massive ambiguity in Lagrangian coordinatization means that there is a corresponding infinite-dimensional family of neutral perturbations in the stress tensor $F$ that do not decay to zero time-asymptotically, but remain constant without affecting the evolution of other, gas-dynamical, variables, and, as a consequence, there can be no coordinate system, extended or otherwise, in which perturbations of $F$ decay. Thus, the strategy followed here will fail; a side-consequence is that gas-dynamical shocks are never asymptotically orbitally stable in Lagrangian coordinates, a fundamental distinction between Eulerian and Lagrangian formulations.

5.3. Possible remedies. The treatment of gas dynamics/MHD is an important direction for future investigation, perhaps by "factoring out" invariances in linearized estimates, then trying to show periodicity modulo these invariant transformations. Another approach might be to treat the problem instead by spatial dynamics techniques plus the standard $O(2)$ reduction argument on the space of time-periodic functions. Here, one must confront similar issues of regularity as faced here, but without having time-evolutionary stability machinery as a guide. A further idea is to carry out a Nash-Moser type iteration in Eulerian coordinates, dealing with loss of regularity in the quasilinear hyperbolic modes by the iteration scheme instead of re-coordinatization.

Finally, an alternative approach modifying our present method would be to establish a "Korntype" inequality showing that there exists a volume-preserving transformation under which $|F|$ is controlled by $|\operatorname{det} F|$ in $H^{s}$ norms. For, we could then carry out our bifurcation analysis in the usual (Eulerian) gas dynamics variables, including $\tau=\operatorname{det} F$, choosing an optimal Lagrangian coordinatization in order to control nonlinear variational estimates on the time- $T$ evolution map. However, we have been unable to establish such an inequality, and suspect that one does not hold. The resolution of this question seems an interesting mathematical problem in its own right.

\section{ApPENDix A. EXPlicit COMPUtATIONS FOR 2D ISENTROPIC VISCOELASTICITY}

In this appendix, we illustrate further the structural assumptions of Section 1.1, suppressing bifurcation parameter $\varepsilon$, by complete computations in a particular case. Expanding coordinatewise the vectorial representation of the equations of isentropic viscoelasticity given in (1.23), in the simple case $d=2$, we obtain

$$
\begin{aligned}
\left(F_{11}\right)_{t}-u_{X}=0, \quad\left(F_{12}\right)_{t}-u_{Y} & =0, \quad\left(F_{21}\right)_{t}-v_{X}=0, \quad\left(F_{22}\right)_{t}-v_{Y}=0, \\
u_{t}-\left(\partial W / \partial F_{11}\right)_{X}-\left(\partial W / \partial F_{12}\right)_{Y} & =\left(\mathcal{Z}_{11}\left(F, \nabla_{X, Y}(u, v)^{T}\right)\right)_{X}+\left(\mathcal{Z}_{12}\left(F, \nabla_{X, Y}(u, v)^{T}\right)\right)_{Y}, \\
v_{t}-\left(\partial W / \partial F_{21}\right)_{X}-\left(\partial W / \partial F_{22}\right)_{Y} & =\left(\mathcal{Z}_{21}\left(F, \nabla_{X, Y}(u, v)^{T}\right)\right)_{X}+\left(\mathcal{Z}_{22}\left(F, \nabla_{X, Y}(u, v)^{T}\right)\right)_{Y},
\end{aligned}
$$

together with compatibility conditions $\partial_{Y} F_{11}-\partial_{X} F_{12}=0, \partial_{Y} F_{21}-\partial_{X} F_{22}=0$. 
A.1. Connection with gas dynamics. In the case $W=W(V)$ of gas dynamics, where $V:=$ $\operatorname{det} F=F_{11} F_{22}-F_{12} F_{21}$ denotes specific volume, the Carnot relation $p d V=d W$ between pressure $p$, specific volume $V$, and elastic potential $W$ (equivalently, work of deformation) yields $p=\frac{d W}{d V}(V)$, from which we recover for appropriate choice of $\mathcal{Z}$ the isentropic version of (5.3), through the computation $\partial W / \partial F_{i j}=(d W / d V)\left(\partial V / \partial F_{i j}\right)=P(V)\left(\partial \operatorname{det} F / \partial F_{i j}\right)$ together with $\partial \operatorname{det} F / \partial F_{11}=$ $F_{22}, \partial \operatorname{det} F / \partial F_{22}=F_{11}, \partial \operatorname{det} F / \partial F_{12}=-F_{21}$, and $\partial \operatorname{det} F / \partial F_{21}=-F_{12}$. Specifically, the choice

$$
\mathcal{Z}(F, Q)=(\operatorname{det} F)\left(\mu \operatorname{Sym}\left(Q F^{-1}\right)+\eta \operatorname{Trace}\left(Q F^{-1}\right)\right) F^{-1, T}
$$

corresponds in Eulerian coordinates to Cauchy stress tensor $T:=(\operatorname{det} F)^{-1} \mathcal{Z} F^{T}=\mu \operatorname{Sym}\left(Q F^{-1}\right)+$ $\eta \operatorname{Trace}\left(Q F^{-1}\right) \mathrm{Id}$, or, substituting $Q=\nabla_{X, Y}(u, v)^{T}$,

$$
T=\mu \operatorname{Sym}\left(\nabla_{x, y}(u, v)^{T}\right)+\eta \operatorname{Trace}\left(\nabla_{x, y}(u, v)^{T}\right) \operatorname{Id},
$$

where $\operatorname{Sym} M:=\frac{1}{2}\left(M+M^{T}\right)$ symmetric part of matrix $M$. This yields in Eulerian coordinates the familiar Navier-Stokes viscosity $\left.\operatorname{div}_{x, y} T=\mu \Delta_{x, y}(u, v)^{T}+(\mu+\eta) \operatorname{div}_{x, y}(u, v)^{T}\right) \operatorname{Id}$ corresponding to the righhand side of (5.3). See [Da, pp. 46-47] for further discussion, along with derivation of (A.3) as the simplest choice satisfying the principles of frame-indifference associated with the problem.

A.2. Parabolicity of viscous terms. Note that (A.1)-(A.2) determine the omitted second-order terms on the righthand side of (5.3). Strict parabolicity of these terms may be deduced either directly, by the evident parabolicity of their Eulerian counterparts together with the Chain Rule, or, appealing to general principles, by verifying the strict Clausius-Duhamel property $Q: \mathcal{Z}(F, Q) \leq$ $-\theta|Q|^{2}<0$ using the strain rate monotonicity criterion of Antman [A]; see [BLeZ, Appendix A.2].

A.3. Hyperbolicity/symmetrizability at endstates. Linearizing about endstates $(F, u)_{ \pm}$firstorder equations (1.28), for $d=2,3$, and writing in vectorial form in terms of unknown

$$
(f, u)=\left(F_{11}, \ldots, F_{1 d}, \ldots F_{d 1}, \ldots F_{d d}, u_{1}, \ldots, u_{d}\right)
$$

we obtain

$$
V_{t}+\sum A^{j} \partial_{j} V=0, \quad A^{j}:=\left(\begin{array}{cc}
0 & E^{j} \\
\left(E^{j}\right)^{T} \frac{d^{2} W}{d f^{2}} & 0
\end{array}\right)
$$

where $E^{1}=\left(\begin{array}{llll}1 & 0 & 0 & 0 \\ 0 & 0 & 1 & 0\end{array}\right)^{T}, E^{2}=\left(\begin{array}{llll}0 & 1 & 0 & 0 \\ 0 & 0 & 0 & 1\end{array}\right)^{T}$ for $d=2$, and similarly for $d=3$. Here, strict rank-one convexity is equivalent to $E(\xi)^{T} \frac{d^{2} W}{d f^{2}} E(\xi)>0$ for all $\xi \in \mathbb{R}^{d}$, where $E(\xi):=\sum_{j} E^{j} \xi_{j}$.

Denoting $A(\xi):=\sum_{j} A^{j} \xi_{j}$, using column operations to expand

$$
\begin{aligned}
\operatorname{det}(A(\xi)-\alpha)=\operatorname{det}\left(\begin{array}{cc}
-\alpha & E(\xi) \\
E(\xi)^{T} \frac{d^{2} W}{d f^{2}} & -\alpha
\end{array}\right) & =\operatorname{det}\left(\begin{array}{cc}
-\alpha & 0 \\
E(\xi)^{T} \frac{d^{2} W}{d f^{2}} & \alpha^{-1} E(\xi)^{T} \frac{d^{2} W}{d f^{2}} E(\xi)-\alpha
\end{array}\right) \\
& =\alpha^{d^{2}-d} \operatorname{det}\left(E(\xi)^{T} \frac{d^{2} W}{d f^{2}} E(\xi)-\alpha^{2}\right)
\end{aligned}
$$

for $\alpha \neq 0$, and extending by analyticity to all $\alpha \in \mathbb{C}$, we find that the eigenvalues of $A(\xi)$ are $\alpha=0, \sqrt{\mu_{k}(\xi)}$, where $\mu_{k}(\xi)$, real, are the eigenvalues of $E(\xi)^{T} \frac{d^{2} W}{d f^{2}} E(\xi)$. Thus, (1.28) is hyperbolic, i.e., $\sigma(A(\xi))$ is real, semisimple, if $W$ is strictly rank-one convex and only if $W$ is rank-one convex.

On the other hand, existence of a block-diagonal symmetrizer $A^{0}=\operatorname{blockdiag}\{M, N\}$ is equivalent to $E(\xi) M \equiv N E(\xi) \frac{d^{2} W}{d f^{2}}$, or $N^{-1} E(\xi) \equiv E(\xi) \frac{d^{2} W}{d f^{2}} M^{-1}$ for all $\xi \in \mathbb{R}^{d}$, or, by linearity:

$$
N^{-1} H \equiv H \frac{d^{2} W}{d f^{2}} M^{-1} \text { for all } H \in \mathbb{C}^{d \times d^{2}}
$$


from which we may deduce that $N=c \mathrm{Id}, M=c \frac{d^{2} W}{d f^{2}}$ for some $c \in \mathbb{C}$. Thus, existence of a blockdiagonal symmetrizer is equivalent to strict convexity of $W$. As noted in Example 1.4, the weaker condition of polyconvexity is sufficient for existence of a $(\bmod m)$ block-diagonal symmetrizer [Da]. As noted in Remark 1.5, rank-one convexity is sufficient for existence of a symmetrizer greater than 0 on ker $m$.

A.4. Explicit $(\bmod m)$ Kawashima skew-symmetrizer. By inspection, the choice

$$
\mathbb{K}_{ \pm}^{j}:=\theta\left(\begin{array}{cc}
0 & \frac{d^{2} W}{d f^{2}} E^{j} \\
-\left(E^{j}\right)^{T} \frac{d^{2} W}{d f^{2}} & 0
\end{array}\right) .
$$

yields for $\mathbb{K}_{ \pm}(\xi):=-\sum_{j} \mathbb{K}_{ \pm}^{j} \xi_{j}$ and $W$ rank one convex at $F_{ \pm}$that, for $\theta>0$ sufficiently small,

$$
(\mathbb{K}(\xi) A(\xi)+\mathbb{B}(\xi))_{ \pm}=\theta\left(\begin{array}{cc}
\frac{d^{2} W}{d f^{2}} \pm(\xi) E(\xi)^{T} \frac{d^{2} W}{d f^{2}} \pm & * \\
* & *
\end{array}\right)+\left(\begin{array}{cc}
0 & 0 \\
0 & \tilde{b}(\xi)
\end{array}\right)
$$

is greater than 0 as a quadratic form when restricted to ker $m$, verifying (A3)(v), by ker $\frac{d^{2} W}{d f^{2}} E \cap$ ker $m=\{0\}$.

A.5. The 1D system. (Verification of (H4)-(H5)) Next, we consider the $1 \mathrm{D}$ version of (1.25):

$$
F_{t}-\left(u_{X}, 0,0\right)=\left(\operatorname{Id}-e_{1} e_{1}^{T}\right) F_{X X}, \quad u_{t}-e_{1}^{T}(d W(F))_{X}=\left(\beta(F) u_{X}\right)_{X},
$$

where $e_{1}=(1,0,0)$ is the first standard basis element. Denoting $\left(a_{1}, a_{2}, a_{3}\right):=\left(F_{11}, F_{21}, F_{31}\right)$ and $\tilde{F}:=\left(\begin{array}{ll}F_{12} & F_{13} \\ F_{22} & F_{23} \\ F_{32} & F_{33}\end{array}\right)$, we find that this decouples into a triangular system consisting of

$$
a_{t}-u_{X}=0, \quad u_{t}-(\partial \omega / \partial a)(a, \tilde{F})_{X}^{T}=\left(\beta(a, \tilde{F}) u_{X}\right)_{X}, \quad \omega(a):=W(F),
$$

together with heat equation $\tilde{F}_{t}=\tilde{F}_{X X}$. For shocks with speed $c \neq 0$, it is readily verified that these equations satisfy the structural assumptions of (A1)-(A3), with $a$ playing the role of hyperbolic variable, and $(\tilde{F}, u)$ of parabolic variable.

Profile equation. Noting that the decoupled $\tilde{F}$ equation does not support nontrivial travelingwave solutions, we find that $\tilde{F} \equiv$ constant for any profile, reducing existence to the corresponding problem for the $1 \mathrm{D}$ reduced equation (1.29) of the introduction, or (A.5) with $\tilde{F}$ held constant.

Eigenvalue equation and verification of $\left(D_{\varepsilon}\right)(\mathrm{ii})$. Likewise, the associated $1 \mathrm{D}$ eigenvalue equations for the linearized equations about a shock profile decouple into

$$
\left(\lambda-c \partial_{x}\right) a-u_{X}=0, \quad\left(\lambda-c \partial_{x}\right) u-(\partial \omega / \partial a)_{X}^{T}=\left(\beta u_{X}\right)_{X},
$$

and $\left(\lambda-c \partial_{x}\right) \tilde{F}=\partial_{X}^{2} \tilde{F}$, hence the associated Evans function factors into the product of the Evans functions for these two systems. But, the $\tilde{F}$ system, being constant-coefficient, is readily seen to have a nonvanishing Evans function in the vicinity of $\lambda=0,{ }^{10}$ hence condition $\left(D_{\varepsilon}\right)$ (ii) reduces to the corresponding condition on the reduced 1D system (1.29) as claimed in the introduction.

\footnotetext{
${ }^{10}$ Specifically, the limits as $\lambda \rightarrow 0$ from the positive real part complex half-plane of its stable manifold as $X \rightarrow$ $+\infty$ / unstable manifold as $X \rightarrow-\infty$ (the limits involved in the definition of the Evans function [GZ, ZH, MaZ]) consist either of solutions blowing up exponentially at the other end $X \rightarrow-\infty / X \rightarrow+\infty$ or of constant solutions that are limits as $\lambda \rightarrow 0$ of solutions blowing up at the other end, either of which prevents their appearance as components of a generalized eigenfunction corresponding to a zero of the Evans function.
} 
A.6. First-order formulation of the eigenvalue equation for $L_{k}(\varepsilon)$. (Verification of (H6)) The eigenvalue equations for the full system (1.25), ignoring constraints, for $d=2$ takes form

$$
\begin{aligned}
\lambda F_{11}-c F_{11}^{\prime}-u_{1}^{\prime} & =i k F_{12}^{\prime}-k^{2} F_{11}, \\
\lambda F_{12}-c F_{12}^{\prime}+i k u_{1} & =F_{12}^{\prime \prime}-i k F_{11}^{\prime}, \\
\lambda F_{21}-c F_{21}^{\prime}-u_{2}^{\prime} & =i k F_{22}^{\prime}-k^{2} F_{21}, \\
\lambda F_{22}-c F_{22}^{\prime}+i k u_{2} & =F_{22}^{\prime \prime}-i k F_{21}^{\prime},
\end{aligned}
$$

together with second-order ODE in $\left(u_{1}, u_{2}\right)$, and similarly for $d=3$. where $c$ is the speed of the shock and "' denotes $\partial_{1}$, Evidently, for $c \neq 0$, these can be rewritten as a first-order system

$$
\begin{aligned}
c F_{11}^{\prime} & =-i k F_{12}^{\prime}+k^{2} F_{11}+-\lambda F_{11}+u_{1}^{\prime}, \\
F_{12}^{\prime} & =F_{12}^{\prime}, \\
F_{12}^{\prime \prime} & =\lambda F_{22}-c F_{22}^{\prime}+i k u_{2},
\end{aligned}
$$

etc. in phase variable $\left(F_{11}, F_{12}, F_{21}, F_{22}, u_{1}, u_{2}, F_{12}^{\prime}, F_{22}^{\prime}, u_{1}^{\prime}, u_{2}^{\prime}\right)$, and similarly for $d=3$.

\section{APPEndix B. JaCOBIAN COMPUTATIONS FOR THE TRUNCATED CUBIC SYSTEM}

In this appendix we carry out the details needed to solve system (4.18), crucial to the proof of our main result. Relabeling slightly for notational convenience, we may write the rescaled system (4.18) as

$$
\left\{\begin{array}{l}
\left(\varkappa \operatorname{sgn}(\varepsilon)+\mathrm{i} \chi \tilde{\mu}+\Lambda|a|^{2}+\Gamma|h|^{2}\right) a=|\varepsilon|^{1 / 2} \Phi_{1}(\varepsilon, \tilde{\mu}, a, h) \\
\left(\varkappa \operatorname{sgn}(\varepsilon)+\mathrm{i} \chi \tilde{\mu}+\Lambda|h|^{2}+\Gamma|a|^{2}\right) h=|\varepsilon|^{1 / 2} \Phi_{2}(\varepsilon, \tilde{\mu}, a, h)
\end{array}\right.
$$

where $\varkappa, \chi$ is constant, $\tilde{\mu}$ is real, $a$ and $h$ complex, and $\Phi_{j} \in \mathbb{C}$ are $C^{1}$ functions, taking without loss of generality (using rotational invariance) $a=\left(a_{1}, 0\right), h=\left(h_{1}, h_{2}\right)$ and assuming the genericity conditions

$$
\Lambda \neq \Gamma, \quad \operatorname{Re}(\Lambda+\Gamma) \neq 0, \quad \operatorname{Re} \Lambda \neq 0 .
$$

Expressed in real coordinates, these are:

$$
\left\{\begin{array}{l}
\left(\varkappa \operatorname{sgn}(\varepsilon)+\operatorname{Re} \Lambda|a|^{2}+\operatorname{Re} \Gamma|h|^{2}\right) a_{1}=|\varepsilon|^{1 / 2} \operatorname{Re} \Phi_{1}(\varepsilon, \tilde{\mu}, a, h), \\
\left(\chi \tilde{\mu}+\operatorname{Im} \Lambda|a|^{2}+\operatorname{Im} \Gamma|h|^{2}\right) a_{1}=|\varepsilon|^{1 / 2} \operatorname{Im} \Phi_{1}(\varepsilon, \tilde{\mu}, a, h), \\
\left(\varkappa \operatorname{sgn}(\varepsilon)+\operatorname{Re} \Lambda|h|^{2}+\operatorname{Re} \Gamma|a|^{2}\right) h_{1}-\left(\chi \tilde{\mu}+\operatorname{Im} \Lambda|h|^{2}+\operatorname{Im} \Gamma|a|^{2}\right) h_{2}=|\varepsilon|^{1 / 2} \operatorname{Re} \Phi_{2}(\varepsilon, \tilde{\mu}, a, h), \\
\left(\varkappa \operatorname{sgn}(\varepsilon)+\operatorname{Re} \Lambda|h|^{2}+\operatorname{Re} \Gamma|a|^{2}\right) h_{2}+\left(\chi \tilde{\mu}+\operatorname{Im} \Lambda|h|^{2}+\operatorname{Im} \Gamma|a|^{2}\right) h_{1}=|\varepsilon|^{1 / 2} \operatorname{Im} \Phi_{2}(\varepsilon, \tilde{\mu}, a, h),
\end{array}\right.
$$

B.1. Case $a_{1} \neq 0, h=0$. Denoting the left-hand side of (B.3) by $H_{1}$, we find easily that at a root $\left(a_{1}, \tilde{\mu}, h_{1}, h_{2}\right)=\left(a_{*}, \tilde{\mu}_{*}, 0,0\right)$ of $(\mathrm{B} .3)$ :

$$
J_{1}:=\left.\operatorname{det} \frac{\partial H_{1}}{\partial\left(a_{1}, \tilde{\mu}, b_{1}, b_{2}\right)}\right|_{\left(a_{*}, \tilde{\mu}_{*}, 0,0,0\right)}=\left|\begin{array}{cccc}
2(\operatorname{Re} \Lambda) a_{*}^{2} & 0 & 0 & 0 \\
2(\operatorname{Im} \Lambda) a_{*}^{2} & \chi a_{*} & 0 & 0 \\
0 & 0 & \operatorname{Re} C_{*} & -\operatorname{Im} C_{*} \\
0 & 0 & \operatorname{Im} C_{*} & \operatorname{Re} C_{*}
\end{array}\right|=2 \chi(\operatorname{Re} \Lambda)(\Lambda-\Gamma) a_{*}^{7},
$$

where $C_{*}:=\varkappa+i \chi \tilde{\mu}_{*}+\Gamma a_{*}^{2}=(\Lambda-\Gamma) a_{*}^{2}$ since $\varkappa+\mathrm{i} \chi \tilde{\mu}_{*}+\Lambda a_{*}^{2}=0$. Thus, under genericity assumptions $\operatorname{Re} \Lambda \neq 0, \Lambda \neq \Gamma$, and since $\chi \neq 0$, by Lemma 4.5, we have $J_{1} \neq 0$ and we can conclude by the Implicit Function Theorem the desired existence and uniqueness of solutions of the $|\varepsilon|^{1 / 2}$-perturbed system nearby those of the $\varepsilon=0$ one. The case $h_{1} \neq 0, a=0$ goes symmetrically. 
B.2. Case $h=a \neq 0$. This case is trickier due to the obvious nonuniqueness induced by rotational invariance of the truncated cubic order system (specifically, the additional rotational invariance in common direction, or $S^{1}$-symmetry). This will require a little bit different handling. Specifically, note that we may by rotational symmetry plus invariance under forward evolution/approximate rotation take both $a$ and $h$ to be real, and work with three unknowns $\left(a_{1}, h_{1}, \tilde{\mu}\right)$ and only three of the equation (B.3). This is enough to give uniqueness of solutions by Implicit Function Theorem, but not existence; existence on the other hand follows by reflective symmetry guaranteeing that $a=h$ is always a solution.

Denoting the first three lines on the left-hand side of (B.3) by $H_{2}$, we have at a root $\left(a_{1}, \tilde{\mu}, h_{1}\right)=$ $\left(a_{\natural}, \tilde{\mu}_{\natural}, a_{\natural}\right)$ of (B.3):

$$
J_{2}:=\left.\operatorname{det} \frac{\partial H_{2}}{\partial\left(a_{1}, \tilde{\mu}, h_{1}\right)}\right|_{\left(a_{\natural}, \tilde{\mu}_{\natural}, a_{\natural}\right)}=\left|\begin{array}{ccc}
2(\operatorname{Re} \Lambda) a_{\natural}^{2} & 0 & 2(\operatorname{Re} \Gamma) a_{\natural}^{2} \\
2(\operatorname{Im} \Lambda) a_{\natural}^{2} & \chi a_{\natural} & 2(\operatorname{Im} \Gamma) a_{\natural}^{2} \\
2(\operatorname{Re} \Gamma) a_{\natural}^{2} & 0 & 2(\operatorname{Re} \Lambda) a_{\natural}^{2}
\end{array}\right|=4 \chi(\operatorname{Re} \Lambda+\operatorname{Re} \Gamma)(\operatorname{Re} \Lambda-\operatorname{Re} \Gamma) a_{\natural}^{5} .
$$

Alternatively, denoting the first, second, and fourth lines of the left-hand side of (B.3) as $H_{3}$, we have

$$
J_{3}:=\left.\operatorname{det} \frac{\partial H_{3}}{\partial\left(a_{1}, \tilde{\mu}, h_{1}\right)}\right|_{\left(a_{\natural}, \tilde{\mu}_{\natural}, a_{\natural}\right)}=\left|\begin{array}{ccc}
2(\operatorname{Re} \Lambda) a_{\natural}^{2} & 0 & 2(\operatorname{Re} \Gamma) a_{\natural}^{2} \\
2(\operatorname{Im} \Lambda) a_{\natural}^{2} & \chi a_{\natural} & 2(\operatorname{Im} \Gamma) a_{\natural}^{2} \\
2(\operatorname{Im} \Gamma) a_{\natural}^{2} & \chi a_{\natural} & 2(\operatorname{Im} \Lambda) a_{\natural}^{2}
\end{array}\right|=4 \chi(\operatorname{Re} \Lambda+\operatorname{Re} \Gamma)(\operatorname{Im} \Lambda-\operatorname{Im} \Gamma) a_{\natural}^{5} .
$$

Under genericity conditions (B.2), one of $J_{2}$ or $J_{3}$ does not vanish, or else $\operatorname{Re}(\Lambda+\Gamma)$ and $(\Lambda-\Gamma)$ would both vanish, a contradiction. But, either of $J_{2} \neq 0$ or $J_{3} \neq 0$ is sufficient to give uniqueness by an application of the Implicit Function Theorem.

Remark B.1. The reason the $h=0$ case works in standard fashion is that the solution is rotationally invariant, so rotation does not induce nonuniqueness. Note that in the $h=0$ case we get both existence- which we need, since it does not follow from invariance considerations- and uniqueness, while for THE $h=a$ case we get only uniqueness? which is all we need, since existence IS guaranteed by reflectional/rotational symmetry (both required for that).

B.3. Case $a=h=0$. Finally, we must treat the trivial solution $(a, h)=(0,0)$. Passing to real and imaginary parts in (B.1) and denoting the left-hand side of this system by $H_{4}$, we find that:

$$
J_{4}:=\left.\operatorname{det} \frac{\partial H_{4}}{\partial\left(a_{1}, a_{2}, h_{1}, h_{2}\right)}\right|_{(0,0,0,0)}=\left|\begin{array}{cccc}
\varkappa & -\chi \tilde{\mu} & 0 & 0 \\
\chi \tilde{\mu} & \varkappa & 0 & 0 \\
0 & 0 & \varkappa & -\chi \tilde{\mu} \\
0 & 0 & \chi \tilde{\mu} & \varkappa
\end{array}\right|=\left(\varkappa^{2}+\chi^{2} \mu^{2}\right)^{2} \neq 0
$$

for arbitrary $\tilde{\mu}$, hence by the Implicit Function Theorem there is a unique solution $(a, b)\left(|\varepsilon|^{1 / 2}, \tilde{\mu}\right)$ for each $(\varepsilon, \tilde{\mu})$ near $\left(0, \tilde{\mu}_{*}\right)$. But, this is already accounted for by the trivial solution $(a, b)=(0,0)$. Thus, we can conclude again in this case the desired existence and uniqueness of solutions of the $\varepsilon$-perturbed system nearby those of the $\varepsilon=0$ one. This completes the argument and the paper.

\section{Appendix C. Alternative treatment of the Constrained Resolvent problem: the METHOD OF FREISTÏHLER-PlAZA}

A subtle point in the analysis was to show that $\{\lambda: \operatorname{Re} \lambda \geq 0\} \backslash\{0\}$ lies in the essential resolvent set of $\left.L\right|_{\text {ker } m}$, done in roundabout fashion by choosing carefully a representative of $L(\bmod m)$ so that $\{\lambda: \operatorname{Re} \lambda \geq 0\} \backslash\{0\}$ lies in the essential resolvent set of the full (unrestricted) operator $L$, then arguing that $(\lambda-L) u=f$ and $f \in \operatorname{ker} m$ implies $u \in$ ker $m$. It is worth mentioning another, more 
direct approach that suffices for systems like isentropic viscoelasticity for which the constraint $m$ arises through reduction from a higher-order equation.

Recall that the linearized equations for isentropic viscoelasticity take the form

$$
\begin{aligned}
\left(\lambda-c \partial_{1}\right) F-\nabla V & =0 \\
\left(\lambda-c \partial_{1}\right) V-\nabla \cdot\left(d^{2} W F\right) & =\nabla \cdot\left(B_{1} \nabla V, \ldots B_{d} \nabla V\right),
\end{aligned}
$$

where $F \in \mathbb{R}^{d \times d}$ and $V \in \mathbb{R}^{d}$, with divergence, gradient, etc., applied row-wise, or, taking Fourier transform in the $x_{2}, \ldots, x_{d}$ directions,

$$
\begin{aligned}
\left(\lambda-c \partial_{1}\right) F-\left(\partial_{1} V, i \tilde{\xi} V\right) & =0 \\
\left(\lambda-c \partial_{1}\right) V-\left(\partial_{1}\left(d^{2} W F\right), i \tilde{\xi}\left(\partial_{1}\left(d^{2} W F\right)\right)\right. & =\partial_{1}\left(B_{1}\left(\partial_{1} V, i \tilde{\xi} V\right)+\sum_{j \neq 1}\left(i \xi_{j} B_{j}\left(\partial_{1} V, i \tilde{\xi} V\right) .\right.\right.
\end{aligned}
$$

Noting by the assumption of parabolicity that $B_{11}$ is invertible, this can be written in standard fashion as a first-order ODE in the phase variable $(F, V, p)$, with $p:=\partial_{1} V$ : the Evans system for (C.1). Modifying a clever observation of $[\mathrm{FP}]$ in the inviscid case, we observe that

$$
F=(Z, i \tilde{\xi} Y), \quad V=(\lambda Y-c Z), \quad p=(\lambda Z-c q)
$$

$Z, V, q \in \mathbb{C}^{d}$, is an invariant subspace of the resulting first-order system, satisfying the reduced equations

$$
Y^{\prime}=Z, \quad Z^{\prime}=q, \quad\left(B_{11} q\right)^{\prime}=h(\lambda, \tilde{\xi}, Y, Z),
$$

where $h$ is linear in $Y, Z$. From (C.3), (C.4), we find that $F=\left(Y^{\prime}, i \tilde{\xi} Y\right)$ is the Fourier transform of a gradient, hence obeys the (Fourier transform version of the) curl free constraint. Moreover, it is readily seen for solutions of (C.2) that at any point $x_{1}$ at which $F$ is a Fourier transform of a gradient, i.e., $F=\left(Y^{\prime}, i \tilde{\xi} Y\right)$, then $(F, V, p)$ lies in this invariant subspace. Hence, ker $m$ is preserved by the flow of the Fourier-transformed eigenvalue equation, and we may construct the resolvent of $\left.L\right|_{\text {ker } m}$ via study of the reduced flow (C.4), for which the properties of hyperbolicity at infinity, etc., follow under the assumption of rank-one convexity of $W$ at endstates $(F, V)_{ \pm}$of the shock. (We shall not carry out these details here, having already constructed the resolvent otherwise.) The above derivation perhaps illuminates a bit the construction of $[\mathrm{FP}]$, at the same time showing its application to general systems obtained by reduction from higher- to lower-order derivative form.

\section{REFERENCES}

[A] S. Antman, Nonlinear problems of elasticity, Applied Mathematical Sciences, 107. Springer-Verlag, New York, 1995. xviii+750 pp. ISBN: 0-387-94199-1.

[B] J.M. Ball, Some open problems in elasticity. Geometry, mechanics, and dynamics, Springer, New York (2002), 3-59.

[BFZ] B. Barker, H. Freistühler, and K. Zumbrun, Convex entropy, Hopf bifurcation, and viscous and inviscid shock stability, preprint (2013).

[BLeZ] B. Barker, M. Lewicka and K. Zumbrun, Existence and stability of viscoelastic shock profiles, Arch. Ration. Mech. Anal. 200 (2011), no. 2, 491-532.

[Ba] G. K. Batchelor, An introduction to fluid dynamics, Cambridge Mathematical Library. Cambridge University Press, Cambridge, paperback edition, 1999.

[BeSZ] M. Beck, B. Sandstede, and K. Zumbrun, Nonlinear stability of time-periodic shock waves, Arch. Ration. Mech. Anal. 196 (2010), no. 3, 1011-1076.

[CK] D. Crawford and E. Knobloch Symmetry and symmetry-breaking bifurcations in fluid mechanics, Ann. Rev. Fluid Mech., 23 (1991) 601-639.

[Da] C. Dafermos, Hyperbolic conservation laws in continuum physics, Springer Verlag 325, Berlin, 2000.

[DM] B. Després and C. Mazeran, Lagrangian gas dynamics in dimension two and Lagrangian systems,

[FP] H. Freistühler and R. Plaza, Normal modes and nonlinear stability behaviour of dynamic phase boundaries in elastic materials, Arch. Ration. Mech. Anal. 186 (2007), no. 1, 1-24. 
[FT] H. Freistühler and Y. Trakhinin, On the viscous and inviscid stability of magnetohydrodynamic shock waves, Phys. D, 237, no. 23 (2008) 3030-3037.

[GZ] R. A. Gardner and K. Zumbrun, The gap lemma and geometric criteria for instability of viscous shock profiles Comm. Pure Appl. Math. 51 (1998), no. 7, 797-855.

[GSS] M. Golubitsky, I. Stewart, D. G. Schaeffer, Singularities and Groups in Bifurcation Theory, Volume II. Springer-Verlag, New York, 1988.

[GMWZ] Gues, O., Metivier, G., Williams, M., and Zumbrun, K., Navier-Stokes regularization of multidimensional Euler shocks, Ann. Sci. École Norm. Sup. (4) 39 (2006), no. 1, 75-175.

[He] D. Henry, Geometric theory of semilinear parabolic equations, Lecture Notes in Mathematics, SpringerVerlag, Berlin (1981).

[HT] D. Hoff and E. Tsyganov, Time analyticity and backward uniqueness of weak solutions of the Navier-Stokes equations of multidimensional compressible flow, J. Differential Equations 245 (2008) 3068-3094.

[Kaw] S. Kawashima, Systems of a hyperbolic-parabolic composite type, with applications to the equations of magnetohydrodynamics. thesis, Kyoto University (1983).

[KaS] Kawashima, S. and Shizuta, Y., On the normal form of the symmetric hyperbolic-parabolic systems associated with the conservation laws, Tohoku Math. J. (1988), 449-464.

[KS] A.R. Kasimov and D.S. Stewart, Spinning instability of gaseous detonations. J. Fluid Mech. 466 (2002), 179-203.

[L] P.D. Lax, Hyperbolic systems of conservation laws and the mathematical theory of shock waves, Conference Board of the Mathematical Sciences Regional Conference Series in Applied Mathematics, No. 11. Society for Industrial and Applied Mathematics, Philadelphia, Pa., 1973. v+48 pp.

[Lun] A. Lunardi, Analytic Semigroups and Optimal Regularity in Parabolic Problems, Birkhäuser Verlag, Basel (1995).

[Ma1] A. Majda, The stability of multi-dimensional shock fronts - a new problem for linear hyperbolic equations. Mem. Amer. Math. Soc. 275 (1983).

[Ma2] A. Majda, The existence of multi-dimensional shock fronts. Mem. Amer. Math. Soc. 281 (1983).

[MaZ] C. Mascia and K. Zumbrun, Pointwise Green's function bounds for shock profiles with degenerate viscosity, Arch. Ration. Mech. Anal. 169 (2003) 177-263.

[M] R. Monteiro, Transverse steady biurcation of viscous shock solutions of a hyperbolic-parabolic model in a strip, Ppreprint (2013).

[SS] B. Sandstede and A. Scheel, Hopf bifurcation from viscous shock waves, SIAM J. Math. Anal. 39 (2008) 2033-2052.

[Se] D. Serre, Systèmes de lois de conservation I-II, Fondations, Diderot Editeur, Paris (1996). iv+308 pp. ISBN: 2-84134-072-4 and xii+300 pp. ISBN: 2-84134-068-6.

[Ta] M. Taylor, Partial Differential Equations III. Applied Mathematical Sciences, Vol. 117. Springer, Heidelberg, 1996.

[Sm] J. Smoller. Shock waves and reaction-diffusion equations. Springer-Verlag, New York, second edition, 1994.

[TZ1] B. Texier and K. Zumbrun, Relative Poincaré-Hopf bifurcation and galloping instability of traveling waves, Methods Anal. and Appl. 12 (2005), no. 4, 349-380.

[TZ2] B. Texier and K. Zumbrun, Galloping instability of viscous shock waves, Physica D. 237 (2008) 1553-1601.

[TZ3] B. Texier and K. Zumbrun, Hopf bifurcation of viscous shock waves in gas dynamics and MHD, Arch. Ration. Mech. Anal. 190 (2008) 107-140.

[TZ4] B. Texier and K. Zumbrun, Transition to longitudinal instability of detonation waves is generically associated with Hopf bifurcation to time-periodic galloping solutions, Comm. Math. Phys. 302 (2011), no. 1, $1-51$.

[Z1] K. Zumbrun, Stability of large-amplitude shock waves of compressible Navier-Stokes equations, With an appendix by Helge Kristian Jenssen and Gregory Lyng. Handbook of mathematical fluid dynamics. Vol. III, 311-533, North-Holland, Amsterdam, (2004).

[Z2] K. Zumbrun, Planar stability criteria for viscous shock waves of systems with real viscosity, in Hyperbolic Systems of Balance Laws, CIME School lectures notes, P. Marcati ed., Lecture Note in Mathematics 1911, Springer (2004).

[Z5] K. Zumbrun, Conditional stability of unstable viscous shock waves in compressible gas dynamics and MHD, Arch. Ration. Mech. Anal. 198 no. 3, 1031-1056.

[Z6] K. Zumbrun, The refined inviscid stability condition and cellular instability of viscous shock waves, preprint (2009).

[ZH] K. Zumbrun and P. Howard, Pointwise semigroup methods for stability of viscous shock waves, Indiana University Journal Vol. 47 (1998), pp. 727-841. 
[ZS] K. Zumbrun and D. Serre, Viscous and inviscid stability of multidimensional planar shock fronts, Indiana Univ. Math. J. 48 (1999), no. 3, 937-992.

MIAMI UNIVERSITY, OXFORD, OH 45056

E-mail address: pogana@miamioh.edu

University OF IowA, IowA City, IA 52242

E-mail address: jinghua-yao@uiowa.edu

INDIANA UNIVERSITY, BLOOMINGTON, IN 47405

E-mail address: kzumbrun@indiana.edu 